\title{
High Energy Physics Division Semiannual Report of Research Activities July 1-December 31, 2004
}

High Energy Physics Division Argonne National Laboratory 


\section{About Argonne National Laboratory}

Argonne is operated by The University of Chicago for the U.S. Department of Energy Office of Science, under contract W-31-109-Eng-38. The Laboratory's main facility is outside Chicago, at 9700 South Cass Avenue, Argonne, Illinois 60439. For information about Argonne and its pioneering science and technology programs, see www.anl.gov.

\section{Availability of This Report}

This report is available, at no cost, at http://www.osti.gov/bridge. It is also available on paper to U.S. Department of Energy and its contractors, for a processing fee, from:

U.S. Department of Energy

Office of Scientific and Technical Information

P.O. Box 62

Oak Ridge, TN 37831-0062

phone (865) 576-8401

fax (865) 576-5728

reports@adonis.osti.gov

\footnotetext{
Disclaimer

This report was prepared as an account of work sponsored by an agency of the United States Government. Neither the United States Government nor any agency thereof, nor The University of Chicago, nor any of their employees or officers, makes any warranty, express or implied, or assumes any legal liability or responsibility for the accuracy, completeness, or usefulness of any information, apparatus, product, or process disclosed, or represents that its use would not infringe privately owned rights. Reference herein to any specific commercial product, process, or service by trade name, trademark, manufacturer, or otherwise, does not necessarily constitute or imply its endorsement, recommendation, or favoring by the United States Government or any agency thereof. The views and opinions of document authors expressed herein do not necessarily state or reflect those of the United States Government or any agency thereof, Argonne National Laboratory, or The University of Chicago.
} 
ANL-HEP-TR-05-10

Argonne National Laboratory

9700 South Cass Avenue

Argonne, Illinois 60439

\section{HIGH ENERGY PHYSICS DIVISION SEMIANNUAL REPORT OF RESEARCH ACTIVITIES}

July 1, 2004 - December 31, 2004

Prepared from information gathered and edited by The Committee for Publications and Information:
Members: P. Malhotra
J. Norem
R. Rezmer
R. Wagner

February 2005 



\begin{abstract}
This report describes the research conducted in the High Energy Physics Division of Argonne National Laboratory during the period of July 1 through December 31, 2004. Topics covered here include experimental and theoretical particle physics, advanced accelerator physics, detector development, and experimental facilities research. Lists of Division publications and colloquia are included.
\end{abstract}





\section{Table of Contents}

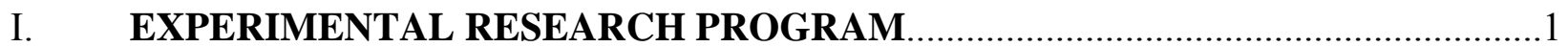

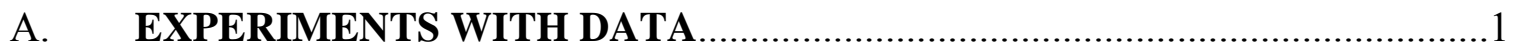

1. Medium Energy Polarization Program ……….........................................1

2. Collider Detector at Fermilab …………..............................................

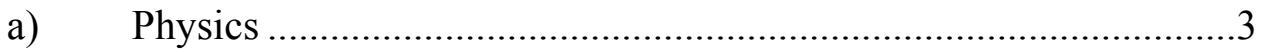

b) Operations and Installation ..........................................................

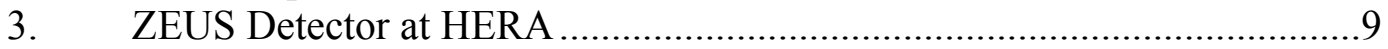

a) Physics Results...........................................................................

b) HERA and ZEUS Operations ……………………………......13

B. EXPERIMENTS IN PLANNING OR CONSTRUCTION ............................13

1. MINOS Main Injector Neutrino Oscillation Search ....................................13

2. ATLAS Calorimeter Engineering Design and Analysis .............................17

a) Mechanical Construction and Installation …………………….......18

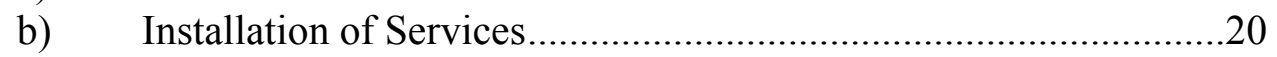

c) Calorimeter Commissioning ………………...............................22

d) Software Development for Tile Commissioning ...........................22

e) Movement System Tests at Argonne .............................................23

f) Movement Control System .........................................................24

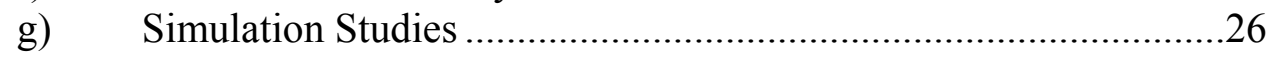

C. NEW PROJECTS AND DETECTOR DEVELOPMENT ............................27

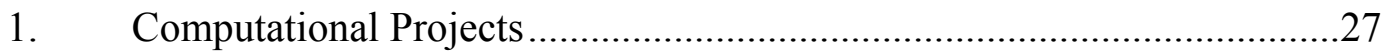

a) ATLAS Computing ...................................................................22

2. Detector Development for the Linear Collider .......................................28

a) Monte Carlo Simulation Studies ....................................................28

b) Development of Resistive Plate Chambers as Active Medium of a Digital Hadron Calorimeter ..................................................29

3. Electronics Support Group.....................................................................33

4. Experiment to Measure the $\theta_{13}$ Neutrino Mass-Mixing Parameter..............38

a) $\quad \mathrm{NO} v \mathrm{~A}$ (NuMI Off-axis $v_{e}$ Appearance) Experiment......................38

b) A New Reactor Neutrino Experiment............................................42

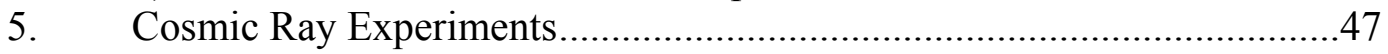

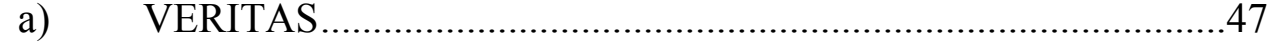

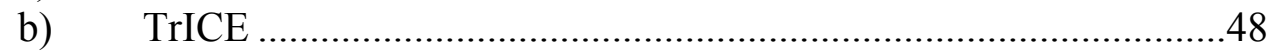

c) The Auger Experiment.....................................................................54

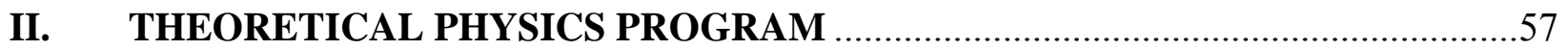

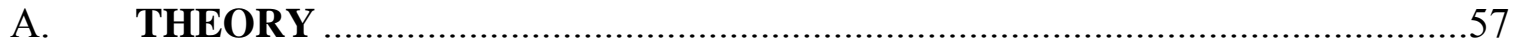

1. Transverse Momentum Distribution of Upsilon Production …………........57

2. Determination of the HWW and HZZ Couplings at the LHC and the ILC ....................................................................................58

3. Bounds on the Cluino Mass from a Global Parton Density Analysis.........58

4. Physics Interplay of the LHC and the ILC...............................................59

5. Lattice Computation of Spin Correlations in NRQCD Color-Octet Matrix Elements. 
6. NRQCD Factorization vs. the Color-Evaporation Model.........................60

7. CERN Yellow Report on Heavy-Quarkonium Physics............................61

8. QWG Topical School on Heavy Quarkonia ..............................................61

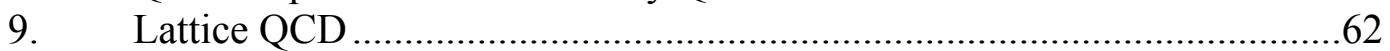

10. Warped Fermions and Precision Tests...............................................63

11. The Supersymmetric Origin of Matter..................................................63

12. Gauged Skyrmions and WZW Interactions in Dimensional Deconstruction .............................................................................66

III. ACCELERATOR RESEARCH AND DEVELOPMENT ....................................6

A. ARGONNE WAKEFIELD ACCELERATOR PROGRAM ........................68

1. The Argonne Wakefield Accelerator Facility Status................................68

2. Development of Externally-Driven, $11.424 \mathrm{GHz}$, Dielectric-Loaded Structures and High-Power Tests at NRL............................................. 71

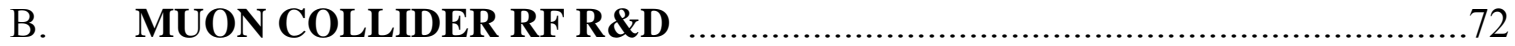

1. High Gradient RF Studies ................................................................. 72

2. Muon Test Area Program.................................................................. 73

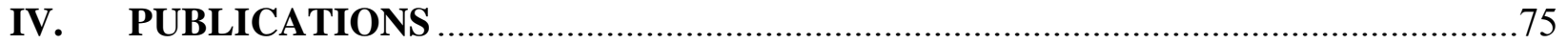

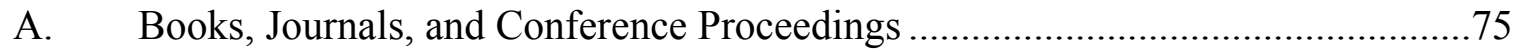

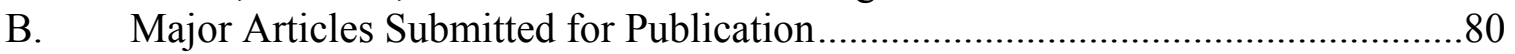

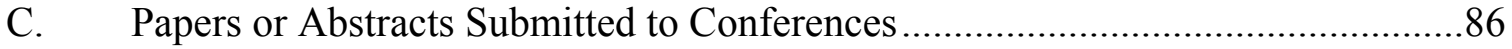

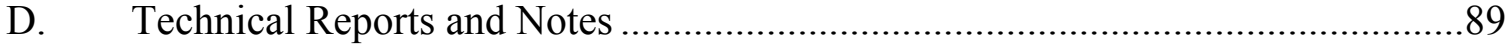

V. COLLOQUIA AND CONFERENCE TALKS .................................................. 91

VI. HIGH ENERGY PHYSICS COMMUNITY ACTIVITIES ...............................98

VII. HIGH ENERGY PHYSICS DIVISION RESEARCH PERSONNEL .....................103 


\section{EXPERIMENTAL RESEARCH PROGRAM}

\section{I.A. EXPERIMENTS WITH DATA}

\section{I.A.1. Medium Energy Polarization Program}

The medium-energy polarization group is primarily interested in the polarized proton program of the STAR experiment at the Relativistic Heavy Ion Collider (RHIC) at Brookhaven. Over the past five years, the majority of our time has been devoted to the construction of a shower maximum detector (SMD) for the STAR Endcap Electromagnetic Calorimeter (EEMC). During the second half of 2004 we worked on a large and diverse group of tasks, however a fundamental goal of most of our efforts was to maximize the physics output of the EEMC and SMD.

The RHIC shutdown which began in May 2004 lasted until November. During this time, electronics for the final two-thirds of the SMD, preshower, and postshower layers were installed and tested. Only one-third of these layers had photomultiplier tubes installed before the 2004 run. We have the complete EEMC for the first time in the FY2005 run, and we plan to commission the new electronics and also make precise measurements of $\pi^{0}$ asymmetries in 2005. Tom Kasprzyk played a significant role in the final assembly of these electronics.

Bob Cadman focused on analysis of data from the 2004 run to extract the analyzing power $\mathrm{A}_{\mathrm{LL}}$ for inclusive $\pi^{0}$ production at high $p_{T}$ using the SMD. A preliminary status report on the analysis was presented to the APS Division of Nuclear Physics at the end of October, and the sensitivity of the algorithm has continued to improve since that meeting. We hope the analysis of 2004 data alone will produce a publishable result, but more significantly the experience we gain will be important for the analysis of run 2005 data. In 2005 we anticipate 60 times more data in the acceptance of the SMD, based on STAR's goal of obtaining 20 times more integrated luminosity (for a total of $7 \mathrm{pb}^{-1}$ ) with the operation of the full SMD. These measurements will be among the first constraints on $\Delta G$, the gluon contribution to the proton spin, to come from RHIC. STAR's ultimate goal is to measure photon-jet coincidences to more directly measure $\Delta G(x)$ over $0.009<x<0.25$, however the time-frame for reaching adequate polarization and luminosity to measure that channel is presently uncertain.

Dave Underwood and Hal Spinka worked on a variety of projects which focused on improving the ultimate systematic precision of polarization data from STAR and RHIC. They have begun to upgrade the STAR Monte Carlo software to improve the modeling of the EEMC response, first by writing software to simulate more accurately the SMD photomultiplier tube response. They are also adding to the simulation package 
a significant amount of material not previously included between the EEMC and the time projection chamber. The additional material may significantly impact the details of showers in the EEMC. Hal Spinka also studied the transverse profiles of the proton bunches in the RHIC ring. Evidence has been found that the polarization decreases near the edges of the bunches, and we will need to understand what effect such a polarization profile will have on measurements of analyzing powers.

An important goal of the RHIC program, following the measurements of $\Delta G$, will be the measurement of parity-violating asymmetries in $W$ production. These asymmetries will allow a separation of the contributions of individual quark and antiquark flavors to the proton spin. At STAR, $W$ bosons will be observed through their decay to high- $p_{T}$ electrons and positrons. The sensitivity to individual flavor contributions is greater when measuring leptons at more forward rapidities, for example in the EEMC. The STAR tracking in this region must be upgraded to achieve adequate discrimination of the charge sign of the high- $p_{T}$ leptons, and therefore our group plans to participate in construction of a GEM detector which will be installed in front of the EEMC. We plan to install a test module prior to the FY2006 run. We have been meeting with collaborators from STAR to plan for this upgrade, and the Argonne group has taken responsibility for the interfacing the front-end electronics of the test module to the existing STAR data acquisition system. Dave Underwood will lead this effort, and he has begun preliminary design work for this system.

During the summer we were visited by Jaki Hostler, a 2004 graduate of Berea College who was looking for an internship related to RHIC before traveling to Germany to study for a master's degree in heavy-ion theory. She analyzed FY2004 data to look for problems in the SMD and studied techniques for calibrating the SMD with $\pi^{0}$ mesons.

We continue to maintain the "E880 polarimeter" in the AGS. The polarimeter will be used again in 2005 to monitor the performance of the AGS components which preserve the proton polarization during the ramp to RHIC injection, including a new cold Siberian Snake which will be installed before the proton run. Hal Spinka continues to serve as co-convenor of the STAR spin physics working group. He also participated in writing a report requested by DOE to present a plan for the RHIC spin program. Work on this report continued into 2005. Finally, during the second half of 2004 the STAR Collaboration published six articles in Physical Review $C$ and one article in Physics Letters.

(R. V. Cadman) 


\section{I.A.2. Collider Detector at Fermilab}

\section{a) Physics}

Fall of 2004 was dominated by a shutdown; data taking was suspended in August and recovery and turning back on started in November. An additional sample of $90 \mathrm{pb}-1$ was collected after the drift chamber recovery, which made a total of $340 \mathrm{pb}-1$ with good drift chamber data; requiring good silicon instead gave samples of $360 \mathrm{pb}-1$. Most analyses used less data. Calibrations were determined for the full dataset and production processing was done and checked.

Our group maintained a strong involvement in $\mathrm{b}$ physics. The study of $\mathrm{B}$ mixing, moving toward the eventual goal of measuring $\mathrm{B}_{\mathrm{s}}$ mixing, continued to make progress. Barry Wicklund and Masa Tanaka collaborated with the CMU group to develop soft electron flavor tagging to use in B mixing studies. The technique was to define an electron likelihood; the calibration of this likelihood is shown in Fig. 1.

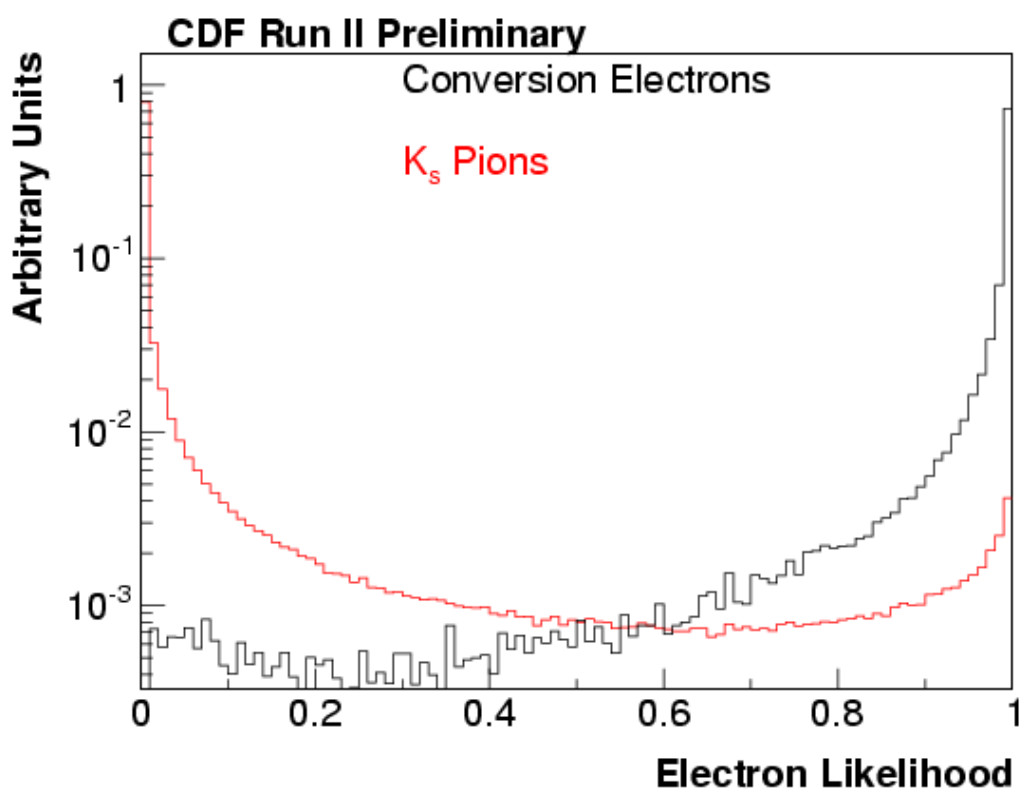

Figure 1. Soft electron tagging uses a combination of variables into a likelihood, which is demonstrated using pure samples of electrons from conversions and pions for $\mathrm{K}$ decay.

A likelihood cut is then used to study tracks in electron and displaced track triggers; tracks which are tagged as electrons may be isolated or associated with a jet with some relative transverse momentum with respect to that jet are then calibrated for their statistical correlation to B flavor ("dilution"), shown in Fig. 2. The product of efficiency times the square of dilution multiplies the number of candidate events to determine the statistical significance of a mixing measurement. It turns out that soft electrons are 
somewhat less effective than soft muons. Masa also continued his work on the fundamental understanding of the semileptonic decay B samples.

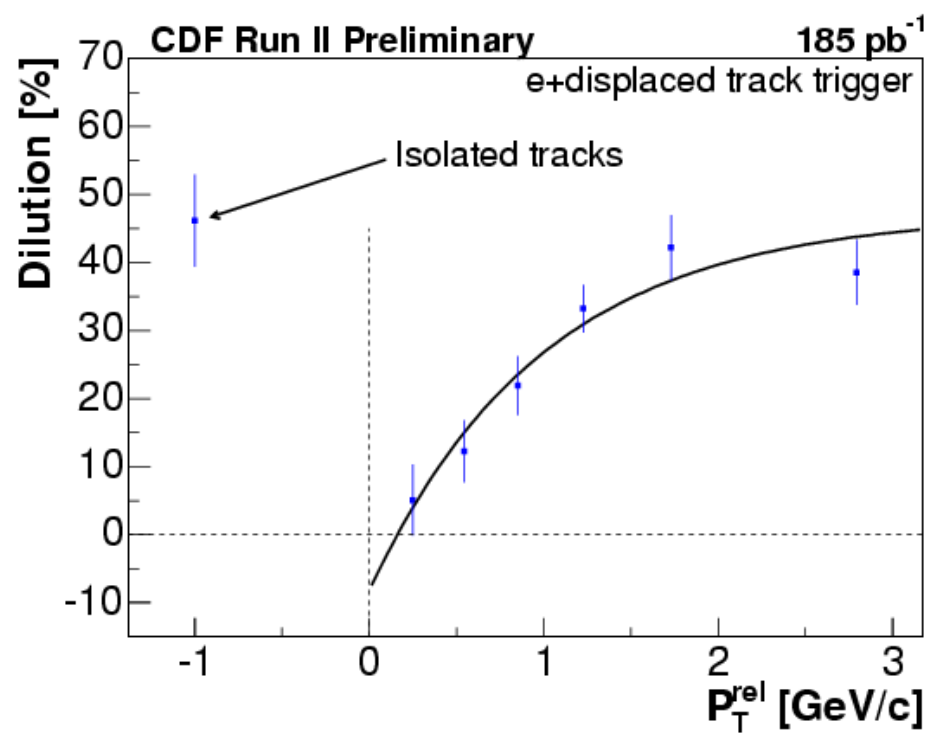

Figure 2. The correlation of an identified (by likelihood) electron with the measured B flavor is used to establish the effectiveness (“dilution") for tagging B flavor using isolated tracks or tags associate with jets.

The $\mathrm{J} / \Psi$ and $\mathrm{B}$ cross section analysis, with Tom LeCompte among the central participants, was submitted for publication and accepted. Another interesting result, which came from Tom's B subgroup, is an attempt to measure the $B_{c}$ meson in exclusive decay. The subgroup agreed on a blind analysis procedure where selection cuts and a statistical indicator of significance were developed in Monte Carlo studies. The idea was to search for the exclusive mode $\mathrm{B}_{\mathrm{c}} \rightarrow \pi \Psi \rightarrow \pi \mu \mu$. A mass peak corresponding to 1 in 1000 for a background fluctuation would be used to allow a peak to determine the mass much more precisely that what came form the observation of $B_{c}$ with three leptons and $a$ are underway to show that the result is indeed the beginning of precision $\mathrm{B}_{\mathrm{c}}$ spectroscopy. Barry was recruited to lead the review of this result moving toward publication. neutrino. "Opening the box" revealed a peak shown in Fig. 3. Further checks on the data are underway to show that the result is indeed the beginning of precision $\mathrm{B}_{\mathrm{c}}$ spectroscopy. Barry was recruited to lead the review of this result moving toward publication. 


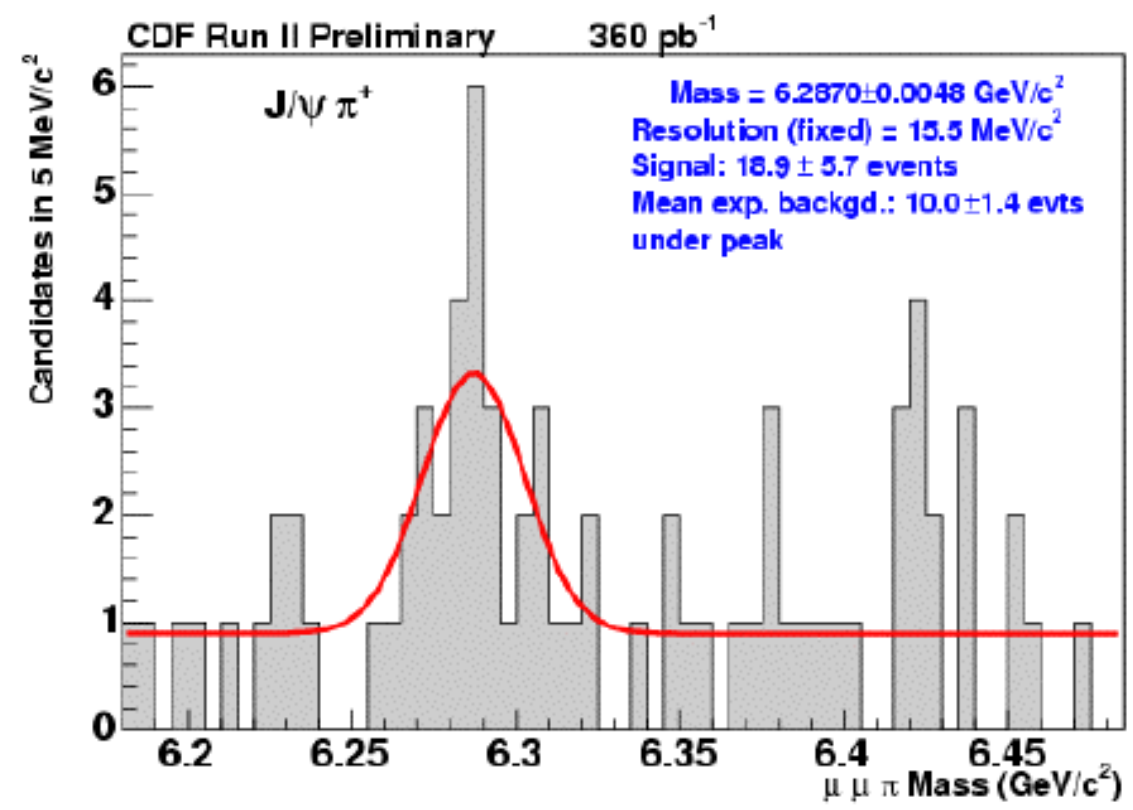

Figure 3. Three prong mass for $B_{c}$ candidates indicating a peak at $6.287 \pm 0.005 \mathrm{GeV} / \mathrm{c}^{2}$.

In electroweak physics, the $\mathrm{W}$ mass group, which includes Larry Nodulman, attempted to produce a preliminary measurement, but were limited to giving public status reports by the delicate and complicated systematic issues. One of these, the linearity of the central EM calorimeter electron measurement, is shown in Fig. 4. It agrees well with the less accurate determination done for the 1994-95 data $\mathrm{W}$ mass.

The template base measurement of the top mass, with Larry leading the internal review, continued to move toward using improved Run II internal jet energy scale determination and improved tracking and silicon B tagging. Steve Kuhlmann has been asked to verify the jet energy scale work.

Progress on photon physics was limited by the distraction of completing, installing and commissioning the new preradiator and crack detectors. 


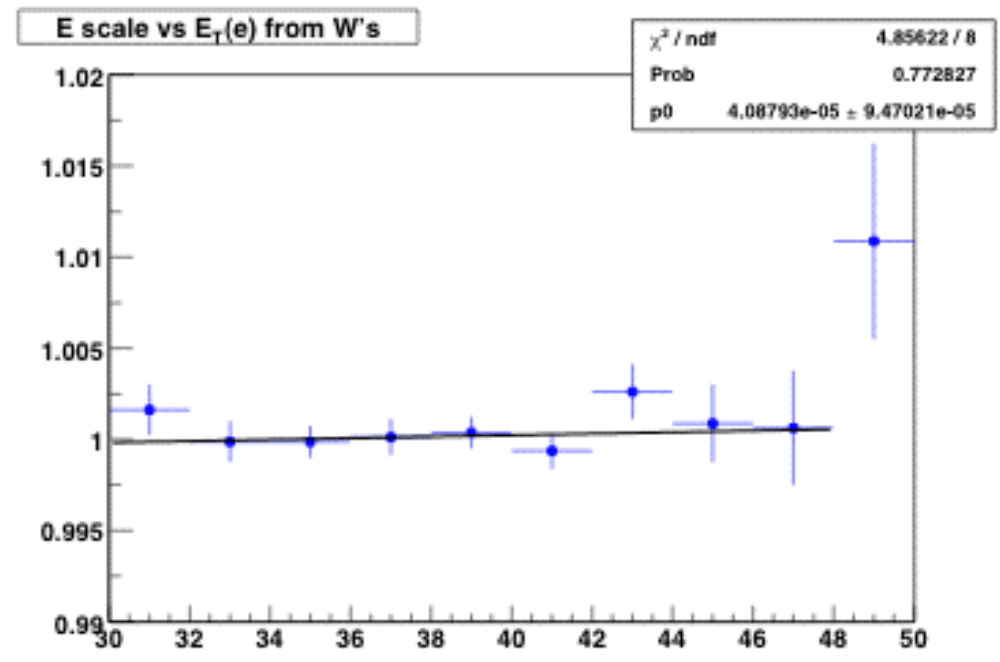

Figure 4. Peak E/P versus energy in $\mathrm{GeV}$, demonstrating a slight upward slope (nonlinearity) similar to what was seen in the 94-95 data.

\section{b) Operations and Installation}

After running through August, the Tevatron shut down for a period to complete the NUMI hookup construction and several other tasks. The running up to the shutdown is summarized in Fig. 6. During the shutdown we installed and checked out the complete preradiator and crack upgrade as well as completing the EM timing installation. The hall was secured in November to get going a data taking resumed in January.

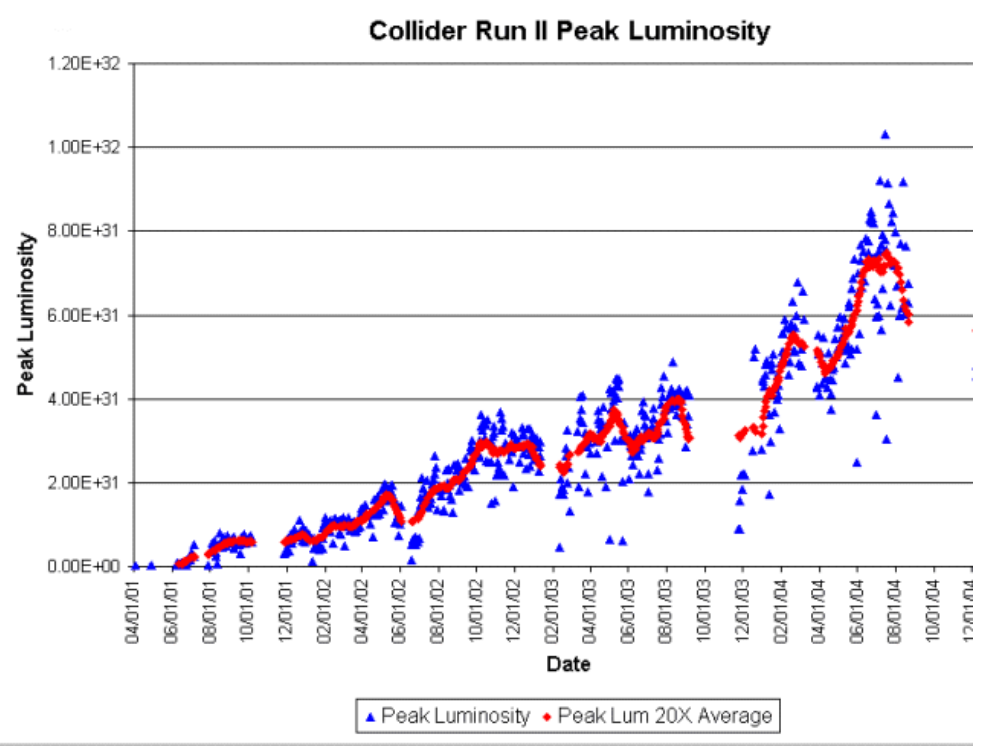

Figure 6. Tevatron Run II peak luminosity for each store and 20 store running average through calendar 2004. 
The installation was accomplished by withdrawing one arch of the calorimeter at a time for access to the front face. The confined space access was used by a crew from various institutions in the U. S., Italy and South Korea to do the calorimeter front face installation, led by Steve Kuhlmann. Changes to the electronics were handled by our Shower Max electronics crew, with help from shift crew recruited from the collaboration, directed by Steve. Fermilab crews took charge of routing fiber bundles alongside the arches and installing PMT boxes. Larry Nodulman monitored the completion of preradiator and crack panels at Argonne and joined the installation crew, which included Jimmy Proudfoot, Karen Byrum and Barry Wicklund. Online software for monitoring was installed and the installations were incrementally checked using cosmic rays taken by shift crew. A cosmic ray occupancy plot taken once the installation was complete is shown in Fig.7. There are three dead channels and a three way cable swap was later discovered in collider data and fixed.

Gain measurements for the PMTs from Tsukuba and Hamamatsu as well as light yields from mapper measurements at Argonne proved reasonably effective at giving a uniform gain, although even without correction, gains were not that bad as can be seen

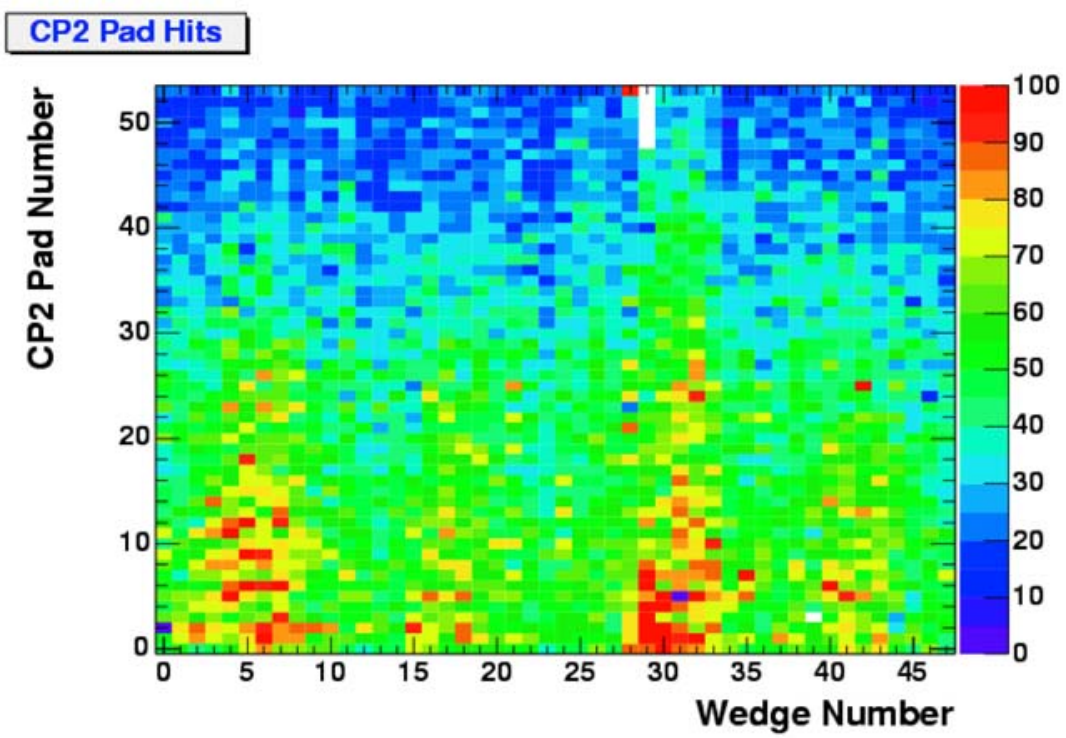

Figure 7. For each of the 48 wedges of the central calorimeter, the cosmic ray occupancy of each of the 54 pads of the upgraded preradiator is shown. Wedge 29 ( 5 east) is the chimney module and is short to the cryostat access.

in the cosmic ray minimum ionizing peak in Fig. 8. The successful completion of this upgrade project was a major accomplishment. 


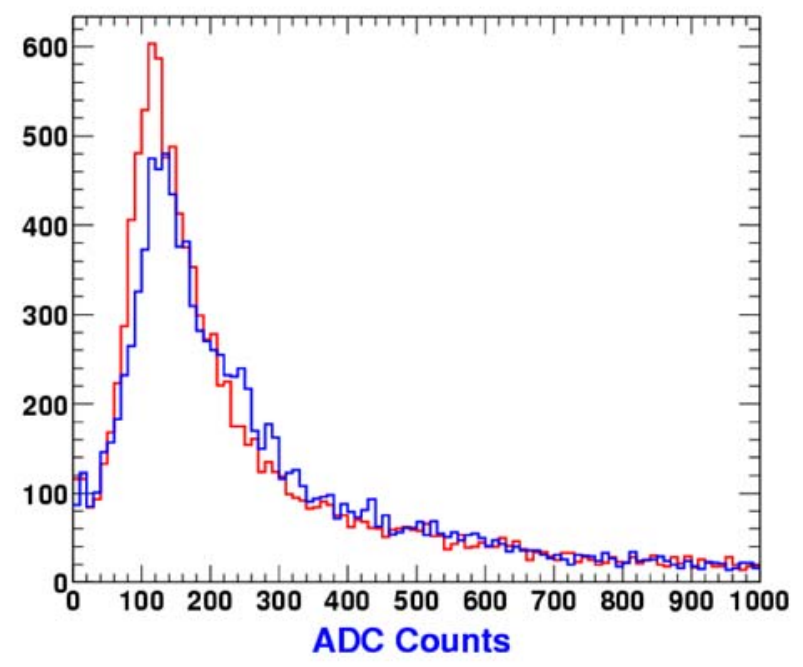

Figure 8. Preradiator upgrade response for upper modules in cosmic rays, with (red) and without (blue) corrections for PMT gain and module maps.

As collider operation was getting started, it was clear that at least one aspect of the upgrade was established. The occupancy in the new detector, read out through those channels which had been used for the old detector, was about five times lower than the expectation for the old detector before the monitoring histogram, as shown in Fig. 9, got updated. Lower occupancy was one of the prime motivations for the upgrade.

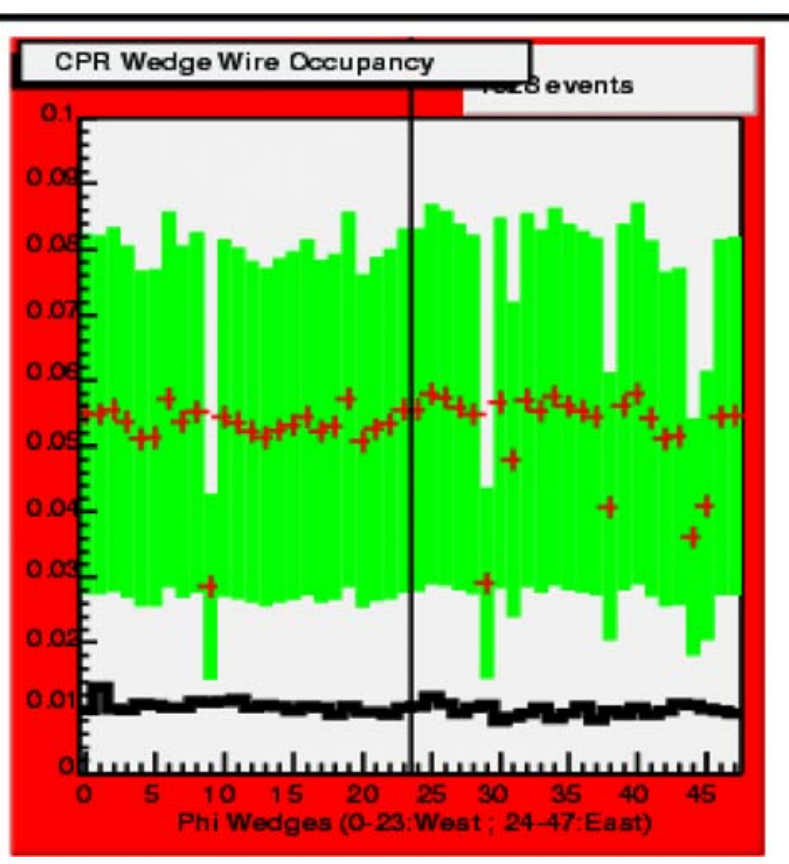

Figure 9. Occupancy of the new preradiator channels using previously used electronic channels us the black histogram, and expectation of occupancy for the previous wire chamber preradiator is the red markers with the green bands. 
As running resumed, Bob Wagner took on the demanding role of operations manager for CDF, Larry Nodulman continued as calorimeter leader, Karen Byrum continued to lead the shower max electronics support and Steve Kuhlmann made sure the upgrade was functioning as well as supporting upgrade and shower max high voltage.

(L. Nodulman)

\section{I.A.3. ZEUS Detector at HERA}

\section{a) Physics Results}

Ten papers were published in this period. In the following, we shall summarize some of these papers.

i) High $Q^{2}$ Neutral Current Cross Sections in $e^{+} p$ Deep Inelastic Scattering at $\sqrt{s}=318 \mathrm{GeV}$

Inclusive cross sections for $\mathrm{e}^{+} \mathrm{p}$ neutral current deep inelastic scattering have been measured at a centre-of-mass energy of $\sqrt{\mathrm{s}}_{\mathrm{s}}=318 \mathrm{GeV}$ using an integrated luminosity of $63.2 \mathrm{pb}^{-1}$. The double differential cross section, $\mathrm{d}^{2} \sigma / \mathrm{dxdQ}^{2}$, has been presented for $200 \mathrm{GeV}^{2}<\mathrm{Q}^{2}<30000 \mathrm{GeV}^{2}$ and for $0.0005<\mathrm{x}<0.65$. The single differential cross sections $\mathrm{d} \sigma / \mathrm{dQ}^{2}, \mathrm{~d} \sigma / \mathrm{dx}$, and $\mathrm{d} \sigma / \mathrm{dy}$ have been presented for $\mathrm{Q}^{2}>200$ $\mathrm{GeV}^{2}$. The effect of $\mathrm{Z}$-boson exchange is seen in $\mathrm{d} \sigma / \mathrm{dx}$ measured for $\mathrm{Q}^{2}>3000 \mathrm{GeV}^{2}$. Figure 1 shows the $\mathrm{e}^{+} \mathrm{p}$ reduced cross section plotted as a function of $\mathrm{x}$ at fixed $\mathrm{Q}^{2}$ between 2000 and $30000 \mathrm{GeV}^{2}$ compared to the e $\mathrm{p}$ reduced cross section presented in a previous ZEUS publication. For $\mathrm{Q}^{2}<3000 \mathrm{GeV}^{2}$ the reduced cross sections are approximately equal. For higher $\mathrm{Q}^{2}$ values the $\mathrm{Z}$-boson exchange contribution causes the $\mathrm{e}^{+} \mathrm{p}$ to be smaller than the e $\mathrm{p}$ cross section. The data presented here were also combined with ZEUS e ${ }^{+} p$ neutral current data taken at $\sqrt{s}_{\mathrm{s}}=300 \mathrm{GeV}$ and the structure function $\mathrm{F}_{2}{ }^{\mathrm{em}}$ was extracted. All results agree well with the predictions of the Standard Model.

ii) Beauty Photoproduction Measured Using Decays into Muons in Dijet Events in ep Collisions at $\sqrt{s}_{\mathrm{s}}=318 \mathrm{GeV}$

The photoproduction of beauty quarks in events with two jets and a muon has been measured using an integrated luminosity of $110 \mathrm{pb}^{-1}$. The fraction of jets containing $b$ quarks was extracted from the transverse momentum distribution of the muon relative to the closest jet. Differential cross sections for beauty production as a function of the transverse momentum and pseudorapidity of the muon, of the associated jet and of $\mathrm{x}_{\gamma}{ }^{\text {jets }}$, the fraction of the photon's momentum participating in the hard process, are compared with Monte Carlo models and QCD predictions made at next-to-leading order. The latter give a good description of the data. Figure 2 shows the differential cross section for b- 
quark production as a function of the b-quark transverse momentum $\mathrm{p}_{\mathrm{T}}{ }^{\mathrm{b}}$ for b-quark pseudorapidity $\left|\eta^{b}\right|<2$ and for $\mathrm{Q}^{2}<1 \mathrm{GeV}^{2}, 0.2<\mathrm{y}<0.8$. The filled points show the results of this analysis and the open point is the previous ZEUS measurement in the electron channel. The dashed line shows the NLO QCD prediction with the theoretical uncertainty shown as the shaded band.

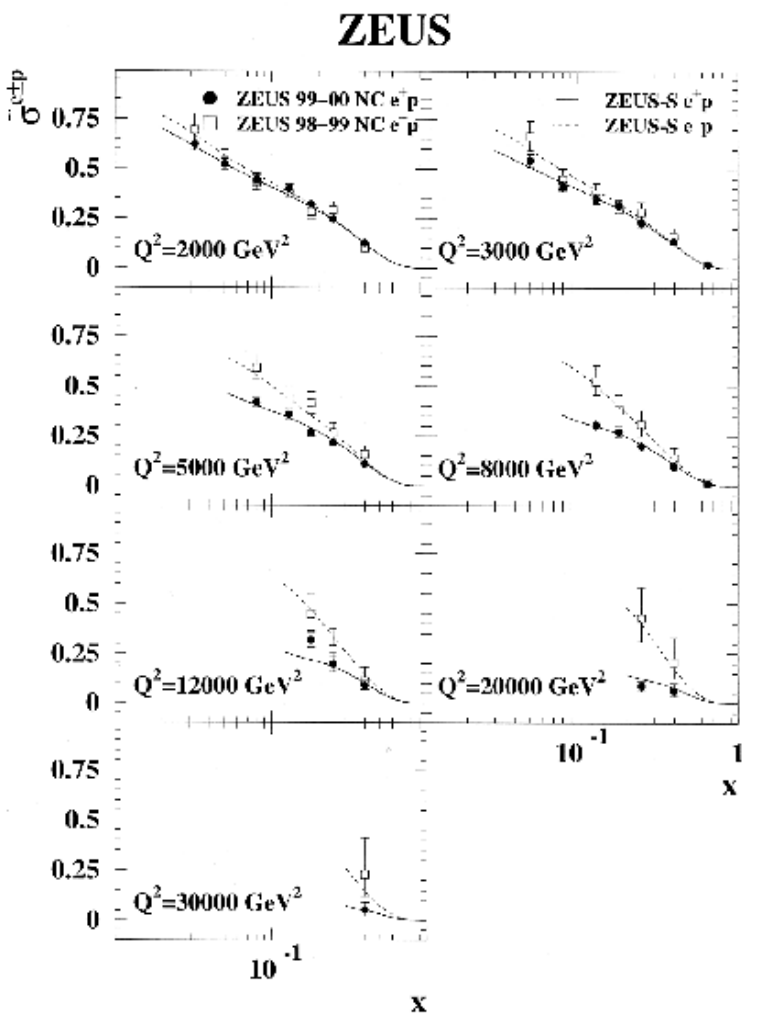

Figure 1. The $\mathrm{e}^{+} \mathrm{p}$ reduced cross section plotted as a function of $\mathrm{x}$ at fixed $\mathrm{Q}^{2}$, compared to the $\mathrm{e}^{-p}$ reduced cross section. The Standard Model expectations, evaluated using the ZEUS-S PDFs, are shown as the solid ( $\left.\mathrm{e}^{+} \mathrm{p}\right)$ and dashed (e-p) lines.

ZEUS

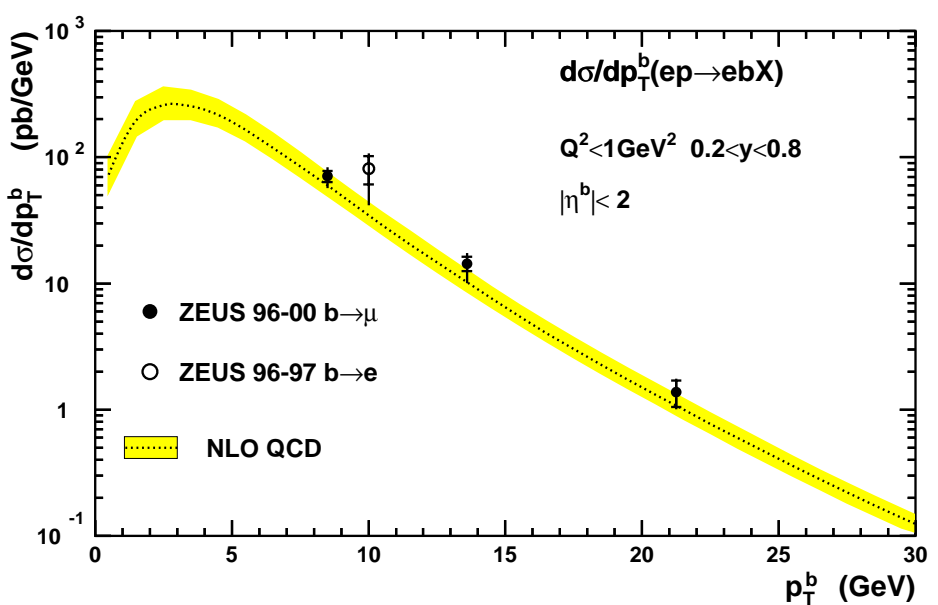

Figure 2. Differential cross section for b-quark production as a function of the b-quark transverse momentum $\mathrm{p}_{\mathrm{T}}^{\mathrm{b}}$ for b-quark pseudorapidity $\left|\eta^{\mathrm{b}}\right|<2$ and for $\mathrm{Q}^{2}<1 \mathrm{GeV}^{2}, 0.2<\mathrm{y}<0.8$. The dashed line shows the NLO QCD prediction with the theoretical uncertainty shown as the shaded band. 
The beauty production cross section for deep inelastic scattering events with at least one hard jet in the Breit frame together with a muon has been measured, for photon virtualities $\mathrm{Q}^{2}>2 \mathrm{GeV}^{2}$, using an integrated luminosity of $72 \mathrm{pb}^{-1}$. The total visible cross section is $\sigma_{b \bar{b}}(\mathrm{ep} \rightarrow \mathrm{e}$ jet $\mu \mathrm{X})=40.9 \pm 5.7$ (stat.) ${ }_{-4.4}^{+6.0}$ (syst.) pb. The next-to-leading order QCD prediction lies about 2.5 standard deviations below the data. The differential cross sections are in general consistent with the NLO QCD predictions; however at low values of $\mathrm{Q}^{2}$, Bjorken $\mathrm{x}$, and muon transverse momentum, and high values of jet transverse energy and muon pseudorapidity, the prediction is about two standard deviations below the data. More data is needed to either confirm or resolve the observed discrepancy between theory and data.

\section{iv) Evidence for a Narrow Baryonic State Decaying to $K_{S}^{0}$ (anti)-proton in Deep Inelastic Scattering at HERA}

A resonance search has been made in the $\mathrm{K}_{S}{ }^{0}$ (anti)-proton invariant-mass spectrum using an integrated luminosity of $121 \mathrm{pb}^{-1}$. The search was performed in the central rapidity region of inclusive deep inelastic scattering at an ep centre-of-mass energy $300-318 \mathrm{GeV}$ for exchanged photon virtuality, $\mathrm{Q}^{2}$, above $1 \mathrm{GeV}^{2}$. Recent results from fixed-target experiments give evidence for a narrow baryon resonance decaying to $\mathrm{K}^{+} \mathrm{n}$ and $\mathrm{K}_{\mathrm{S}}^{0} \mathrm{p}$, interpreted as a Pentaquark. The results presented here support the existence of such a state, with a mass of $1521.5 \pm 1.5$ (stat.) ${ }_{-1.7}^{+2.8}$ (syst.) $\mathrm{MeV}$

\section{ZEUS}

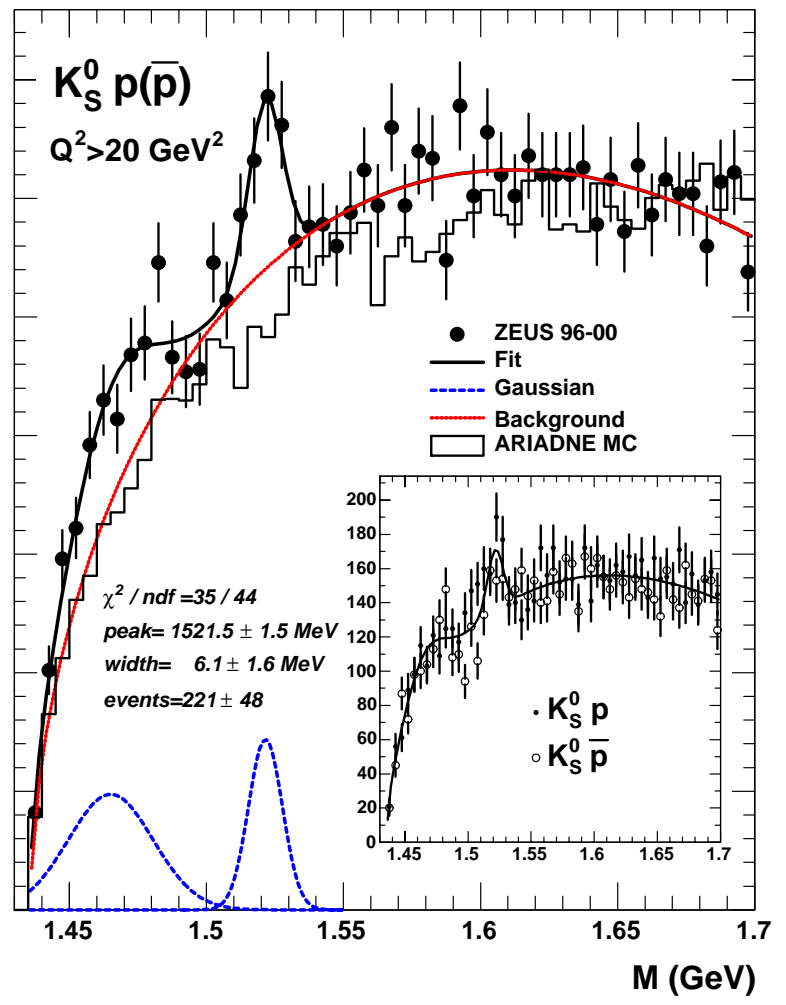

Figure 3. The invariant spectrum for the $\mathrm{K}_{\mathrm{S}}^{0}$ (anti)-proton channel for $\mathrm{Q}^{2}>20 \mathrm{GeV}^{2}$. The solid line is the result of a fit to the data using a three-parameter background function plus two Gaussians. The histogram shows the prediction of the ARIADNE Monte Carlo simulation normalized to the data in the mass region above $1650 \mathrm{MeV}$. The inset shows the $\mathrm{K}_{\mathrm{S}}^{0}$ proton and $\mathrm{K}_{\mathrm{S}}{ }^{\mathrm{a}}$ antiproton candidates separately, compared to the result of the fit for the combined sample scaled by a factor of 0.5 
and a Gaussian width consistent with the experimental resolution of $2 \mathrm{MeV}$. Figure 3 shows the invariant mass spectrum for the $\mathrm{K}_{\mathrm{S}}{ }^{0}$ (anti)-proton channel for $\mathrm{Q}^{2}>20 \mathrm{GeV}^{2}$. the solid line is the result of a fit to the data using a three-parameter background function plus two Gaussians, centered at $1465.1 \pm 2.9$ (stat.) and $1521.5 \pm 1.5$ (stat.) MeV. The signal at $1521.5 \mathrm{MeV}$ contains $221 \pm 48$ events. The probability of a similar signal anywhere in the range $1500-1560 \mathrm{MeV}$ arising from fluctuations of the background is below $6 \times 10^{-5}$. Monte Carlo simulations based on the ARIADNE program feature no significant structure in the $\mathrm{K}_{\mathrm{S}}{ }^{0} \mathrm{p}$ mass range of 1.45 to $1.7 \mathrm{GeV}$.

\section{v) Search for a Narrow Charmed Baryonic State Decaying to $D^{{ }^{*}} p^{\mp}$ in ep Collisions at HERA}

A resonance search has been made in the $\mathrm{D}^{* \pm} \mathrm{p}^{\mp}$ invariant-mass spectrum using an integrated luminosity of $126 \mathrm{pb}^{-1}$. The decay channels $\mathrm{D}^{*+} \rightarrow \mathrm{D}^{0} \pi_{\mathrm{s}}^{+} \rightarrow\left(\mathrm{K}^{-} \pi^{+}\right) \pi_{\mathrm{s}}^{+}$and $\mathrm{D}^{*+} \rightarrow \mathrm{D}^{0} \pi_{\mathrm{s}}^{+} \rightarrow\left(\mathrm{K}^{-} \pi^{+} \pi^{+} \pi^{-}\right) \pi_{\mathrm{s}}^{+}$(and the corresponding antiparticle decays) were used to identify $\mathrm{D}^{{ }^{* \pm}}$ mesons. No resonance structure was observed in the $\mathrm{D}^{{ }^{*}} \mathrm{p}^{\mp}$ mass spectrum from more than 60000 reconstructed $\mathrm{D}^{{ }^{* \pm}}$ mesons. The results are not compatible with a report of the $\mathrm{H} 1$ collaboration of a charmed Pentaquark with a mass around $3099 \mathrm{MeV}$. Figure 4 shows the mass distribution of $\mathrm{D}^{*} \mathrm{p}$ for both $\mathrm{D}^{*}$ decay channels separately. The data samples containing deep inelastic scattering events are shown in the lower two plots. The shaded histograms on top of the background fit correspond to the expected rate of charmed Pentaquark events in accordance with the results of the H1 collaboration.

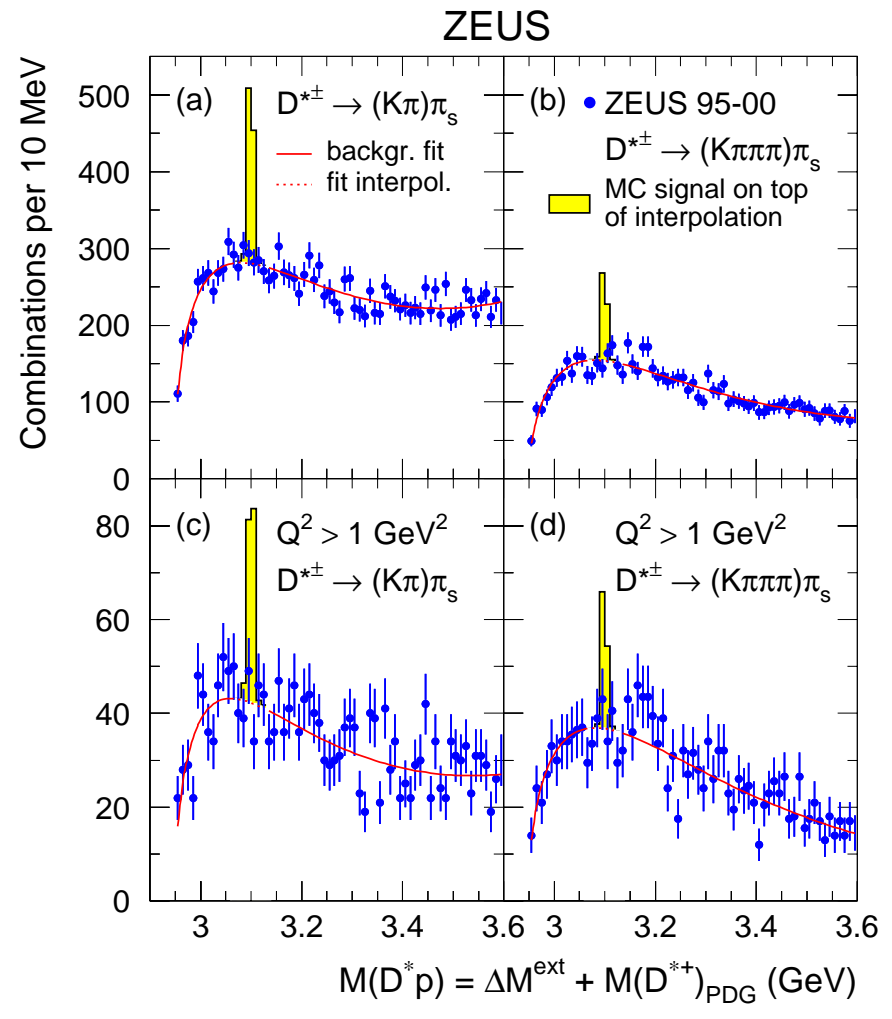

Figure 4. The distribution of $M\left(D^{*} p\right)$ for charmed Pentaquark candidates selected in (a) the full data sample using $\mathrm{D}^{* \pm} \rightarrow$ $(\mathrm{K} \pi) \pi_{\mathrm{s}}$ candidates, (b) the full data sample using $\mathrm{D}^{* \pm} \rightarrow$ $(\mathrm{K} \pi \pi \pi) \pi_{\mathrm{s}}$ candidates, (c) DIS with $\mathrm{Q}^{2}>1 \mathrm{GeV}^{2}$ using $\mathrm{D}^{* \pm} \rightarrow$ $(\mathrm{K} \pi) \pi_{\mathrm{s}}$ candidates and (d) DIS with $\mathrm{Q}^{2}>1 \mathrm{GeV}^{2}$ using $\mathrm{D}^{{ }^{*}} \rightarrow$ $(\mathrm{K} \pi \pi \pi) \pi_{\mathrm{s}}$ candidates. The solid curves are fits to the background function outside the signal window of $3.07-3.13$ $\mathrm{GeV}$. The shaded histogram shows the Monte Carlo predictions of a charmed Pentaquark assuming the mass and rates published by the $\mathrm{H} 1$ collaboration. 


\section{b) HERA and ZEUS Operations}

In the second half of 2004 HERA continued its positron run delivering a total integrated luminosity of $78 \mathrm{pb}^{-1}$ in 2004 . The background conditions were excellent. The positrons at the interaction region were either right-hand or left-hand polarized.

After the summer shutdown the machine converted to electron - proton collisions. After initial struggles the machine was able to deliver acceptable collision rates. The run will continue virtually uninterrupted until the summer shutdown of 2005 .

The ZEUS detector is performing well. Due to a heating problem creating a leak in the superconducting coil, the collaboration decided in October to momentarily switch off the Straw Tube Tracker. Considerable effort is being dedicated to understand the source of the heat and to finding ways to remove it.

(J. Repond)

\section{I.B. EXPERIMENTS IN PLANNING OR CONSTRUCTION}

\section{I.B.1. MINOS - Main Injector Neutrino Oscillation Search}

The phenomenon of neutrino oscillations allows the three flavors of neutrinos to mix as they travel through space or matter. The MINOS experiment will use a Fermilab muon neutrino beam to study neutrino oscillations with higher sensitivity than any previous experiment. MINOS is optimized to explore the region of neutrino oscillation parameter space (values of the $\Delta m^{2}$ and $\sin ^{2}(2 \theta)$ parameters) suggested by atmospheric neutrino experiments. To accomplish this, the experiment compares the rates and characteristics of neutrino interactions in a 980-ton "near" detector, close to the source of neutrinos at Fermilab, and a 5400-ton "far" detector, $735 \mathrm{~km}$ away in the underground laboratory at Soudan, Minnesota. The MINOS detectors are steel-scintillator sandwich calorimeters with toroidally magnetized 1-inch thick steel planes. The detectors use extruded plastic scintillator with fine transverse granularity (4-cm wide strips) to provide both calorimetry (energy deposition) and tracking (topology) information. The neutrino beam and MINOS detectors are being constructed as part of the NuMI (Neutrinos at the Main Injector) Project at Fermilab.

Argonne physicists and engineers have been involved in many aspects of MINOS construction during the past decade: scintillator-module factory engineering, near-detector scintillator-module fabrication, near-detector front-end electronics, nearand far-detector installation, detector and electronics energy calibration in a test beam at CERN and the construction and installation of neutrino beamline components. Argonne 
physicists are now working with groups from Indiana University and the University of Minnesota to measure the ratio of positive and negative cosmic-ray muon fluxes with data from the MINOS far detector. Finally, since 2002 the group has been devoting considerable effort to the design of next-generation neutrino experiments, as described in the Detector Development section of this report.

MINOS detector work achieved several important milestones during the second half of 2004. The Argonne group members continued to play major roles at Fermilab, where the installation of MINOS near detector planes, electronics and data acquisition system was completed in August. Argonne physicists and engineers have been heavily involved in the installation and commissioning of all near detector systems. The Argonne electronics group completed the fabrication of front-end electronics in 2003 and finished the associated checkout in early 2004. The group's work to implement various electronics modifications during detector commissioning is now nearly complete. The installation of the near detector magnet coil was finished in September. The power supply and coil connections were completed in November and the coil was energized for the first time in December. Figure 1 is a photograph of the near detector taken just after the coil was installed and connected. The near detector recorded a large sample of cosmic-ray events for alignment purposes before the steel planes were magnetized and is now routinely recording cosmic-ray-muon calibration data. Although there are still a

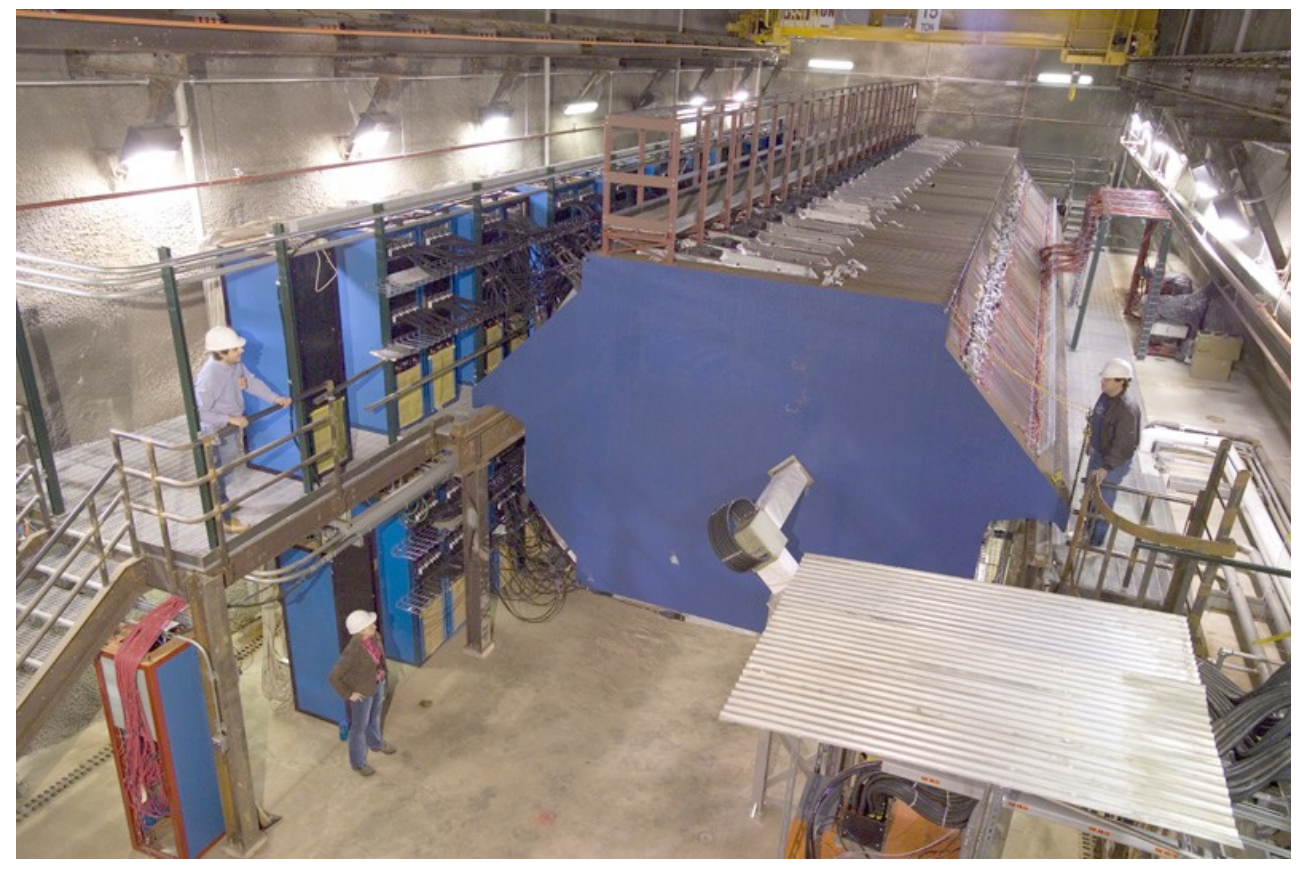

Figure 1. The completed MINOS near detector. The 980-ton detector consists of 280 one-inch thick steel planes that are toroidally magnetized by a 40,000 Amp-turn coil. The detector is instrumented with 153 planes of $4-\mathrm{cm}$ wide plastic scintillator strips that are interleaved with the steel planes. The photograph shows the upstream end of the detector after the magnetic coil was installed in the channel through the steel and scintillator planes. The beam is approximately centered between the coil and the left-hand (west) edge of the first plane. 
number of minor problems to be resolved, the near detector is now ready to record the first neutrino interactions from the NuMI beam, which is scheduled to be turned on for the first time in January 2005.

At Soudan, the far detector continued to record high quality data on cosmic-ray muons and atmospheric-neutrino interactions, with an average duty cycle exceeding 95\%. As described in previous reports, the magnetization of steel planes in the MINOS far detector enables a unique search for CPT symmetry violation in atmospheric neutrino oscillations. During a five-year exposure, which began in 2003, the experiment will search for CPT-violating differences in the oscillations of muon neutrinos and muon antineutrinos. The MINOS far detector is the only atmospheric neutrino experiment able to distinguish between positive and negative muons from charged current neutrino interactions. Finally, the decommissioning and removal of the Soudan 2 detector from the Soudan underground laboratory began in late 2004. The detector ceased operation in July 2001 to save operating funds.

Since 2002, Argonne physicists have worked on the construction and testing of NuMI neutrino beam components at Fermilab. One physicist is the Deputy Level 3 Manager for Neutrino Beam Devices and is responsible for preparation of the work cell facility, which will be used to repair highly radioactive beamline components. Other group members worked on remote-control rigging procedures, vibration measurements of the production target and magnetic focusing horns, horn magnetic-field measurements and the readout and integration of target hall instrumentation. The paragraphs below describe Argonne achievements in these areas during the second half of 2004.

The most complex part of the work cell is the remotely controlled lift-table system, which is used inside a shielded enclosure to attach or detach a target or horn from its 26-ton steel support module. The system was used to attach the production target and the two magnetic focusing horns to their support modules prior to initial installation in the target chase. In September, the target hall crew installed lead-glass blocks in the four viewing windows in the work cell walls, completing the work cell construction. In December, the work cell was the focus of hot handling practice, during which the target hall crew tested and documented the remote-control techniques for removing highly radioactive horn and target assemblies from the target chase and replacing them with new units in the work cell. Figure 2 shows the target-baffle carrier in the work cell, supported by the lift tables, just before it was attached to the support module. The hot-handling practice was about $50 \%$ complete at the end of the month. 


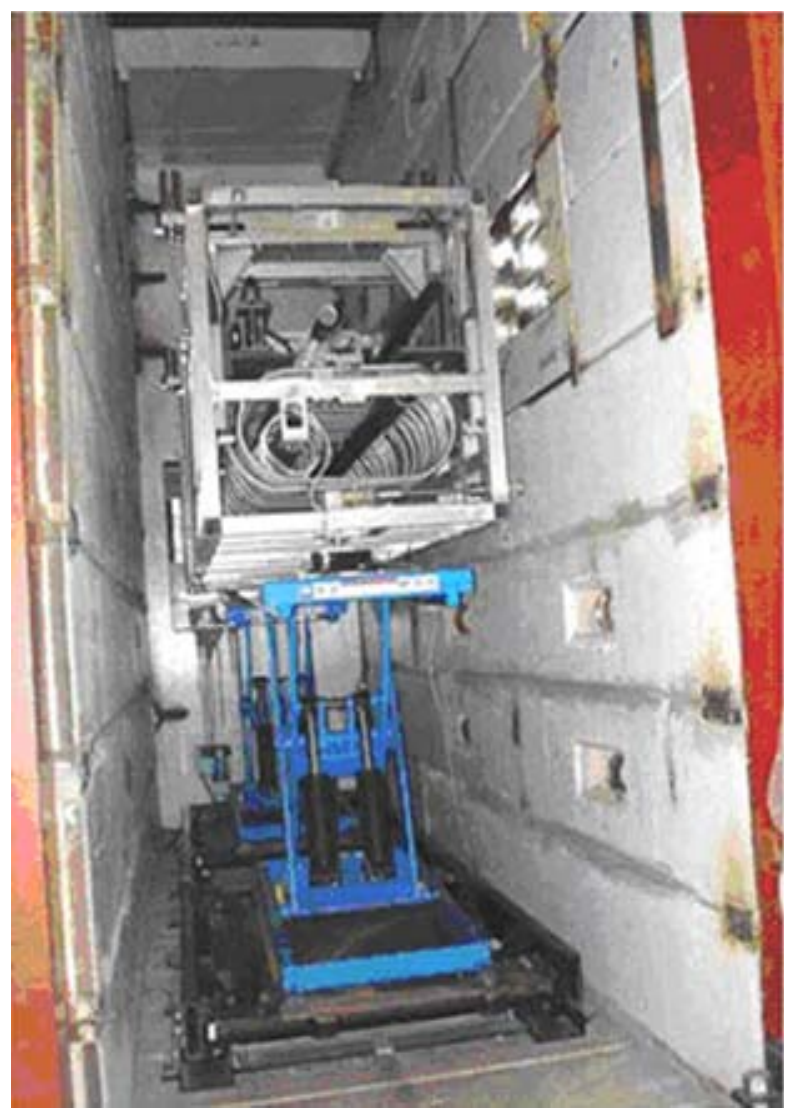

Figure 2. The NuMI target-baffle carrier in the work cell. The work cell is used to attach and detach the target-baffle carrier assembly, which will eventually become intensely radioactive, from its massive support module. The photograph, taken through the (open) remotely movable $1-\mathrm{ft}$ thick steel shield door, shows the upstream (baffle) end of the carrier before the support module is lowered into place above it. Operators can view the operation through lead-glass windows as they position the carrier and connect it to the support module. One of the windows can be seen in the photograph, near the top right side of the carrier, which is supported by the lift tables.

Argonne physicist Bob Wagner is responsible for the installation of cameras and other equipment needed for remote control of the target hall bridge crane during hot handling. These will be used to move radioactive shield blocks and the target and horn subassemblies if faults occur in these components after initial operation of the high intensity proton beam. Failed components, still attached to their support modules, will be moved into the work cell, where they will be repaired or replaced. These hot handling procedures were tested under realistic conditions for the first time in December, when shield blocks were removed from the target chase and target assembly was removed from the chase and placed in the work cell by remote control. The camera system and procedures all performed well after some minor modifications. Hot handling practice will be completed in January 2005 when procedures for horn replacement will be tested.

Argonne physicists and engineers also continued work on the preparation and checkout of the electronics used to read out target hall instrumentation. Argonne's instrumentation readout responsibilities include the thermocouples that measure temperatures at many locations in the target hall, the monitors for horn magnetic fields and horn power supply current, the beam-loss monitors used for horn-crosshair alignment measurements, the readout of the precise positions of the target carrier and the first horn, and the radiation-hard instrumentation cabling. Figure 3 is a photograph of the downstream end of the first horn installed in the target chase, showing the alignment crosshair and the beam loss monitor. In November, the horns were pulsed to full current 


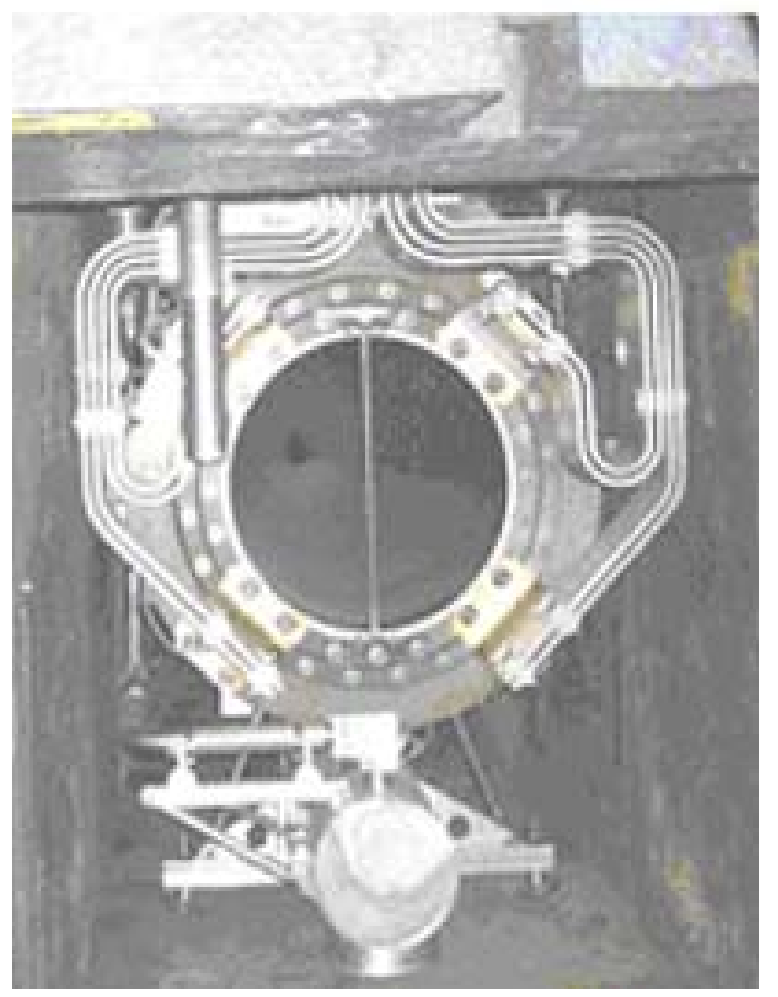

Figure 3. Horn 1 alignment crosshair and beam loss monitor installed in the target chase. The photograph shows the downstream end of the first horn with the 200,000 Amp stripline connections and the beamalignment crosshair. The beamloss monitor is the cylindrical object protruding through the top shielding to the left of the horn. The counting rate in the monitor increases as the proton beam is scanned across the aluminum crosshair, which is located a few millimeters away from the beam centerline (to protect it during high intensity operation).

for the first time in the target hall, allowing the initial checkout of horn instrumentation readout. In early December, the Argonne group completed the fabrication of signal conditioning electronics, which is used to interface instrumentation signals to the ACNET data acquisition system, and began commissioning of this system in the target hall. Also in December, the Main Injector extracted the first protons to the NuMI beamline, which transported them through the target hall and decay pipe to the hadron absorber. This important milestone demonstrated the proper alignment of beamline magnets and instrumentation, and provided the first test of the beam-loss monitors used for horn crosshair alignment. After some minor modifications, all systems are expected to be ready for the first production of neutrinos from the NuMI target in mid January.

(D.S. Ayres)

\section{I.B.2. ATLAS Calorimeter Engineering Design and Analysis}

\section{Tile Calorimeter Construction and Installation}

There were 2 major accomplishments in the second half of 2004. First, the dummy load test on the EBA cylinder was completed. This has provided crucial information for the preparation for the final assembly of the Extended Barrel 
calorimeters, which is to occur towards the end of 2005. Second, the installation of the barrel calorimeter was completed on Dec $10^{\text {th }}$, a major milestone for ATLAS, and we are moving forward to the next phases which include the installation of services and electronics commissioning. Work also progressed in areas looking forward to operation of the calorimeter (installation of services, commissioning and simulation studies.) Finally, the Tile Calorimeter subsystem decided to instrument one of the additional modules. ANL65 was used as the absorber and an ANL technician carried out the majority of the work needed to insert tiles and insert, route and glue the readout fibers.

\section{a) Mechanical Construction and Installation}

\section{EBA pre-assembly and cryostat dummy load test}

Due to the unique way in which the Endcap Cryostat load is supported by the Extended Barrel Tile Calorimeter, it was necessary to perform a full load test to demonstrate the stability of the system as well as to establish limits on the deflections of the cylinder. An Argonne engineer was responsible for defining the parameters of this test, monitoring it and evaluating the results.

Prior to conducting this test both 2D and 3D engineering analyses had been conducted. The 3D FEA model showed that the structure was stable. However the 2D static model showed that potential failure scenarios existed. Therefore, to provide additional safety during the load transfer of the cryostat to the EB a chain was connected the outer surfaces of the saddle support beams together (ANL Tech Note: ANL-HEP-TR04-65.) This chain will only be under load if a problem occurs and was instrumented with a load cell to monitor the force on it. The cryostat dummy load was applied in stages and the force on the chain was monitored throughout the loading process.

The force on the chain increased by $\sim 0.3$ tons from the beginning to the end of the loading (this was most likely caused by the rotation of the saddles.) After the loading was completed a quarter turn was applied to the swivel hook attaching the chain to the saddle and load returned to its original value (without Dummy load.) This confirmed one crucial conclusion of the FEA analysis - namely that the system was mechanically stable. The FEA calculation had also predicted a loss of contact on the lower swivel bolts. This was also observed during the load test (the bolts were loose and could be turned by hand.)

Deflection data was also taken during this test. Since deformation of the shims at the inner radius was anticipated, these results were evaluated by comparing a difference of differences:

$$
\text { [FEA(noload })- \text { FEA(load) }]-[(\text { Test(Noload })-\text { Test(load })]
$$




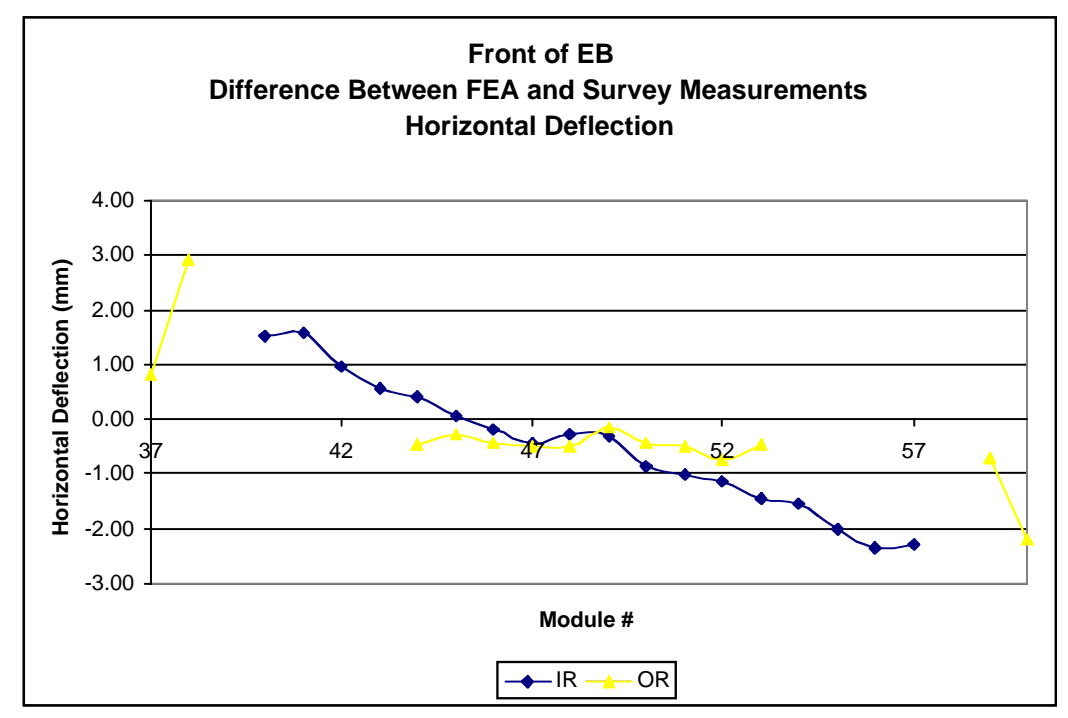

Figure 1. Comparison of the FEA calculation with measurements made during the dummy load test, as described in the text.

A typical plot (in this case the horizontal deformation) is shown in figure 1. The FEA predictions of module deflections were consistent with the survey results and were within a few $\mathrm{mm}$. They are, however, sufficiently different that we will be required to compensate for them during the final assembly. In addition, we observed much better

\section{Barrel Calorimeter Final Assembly}

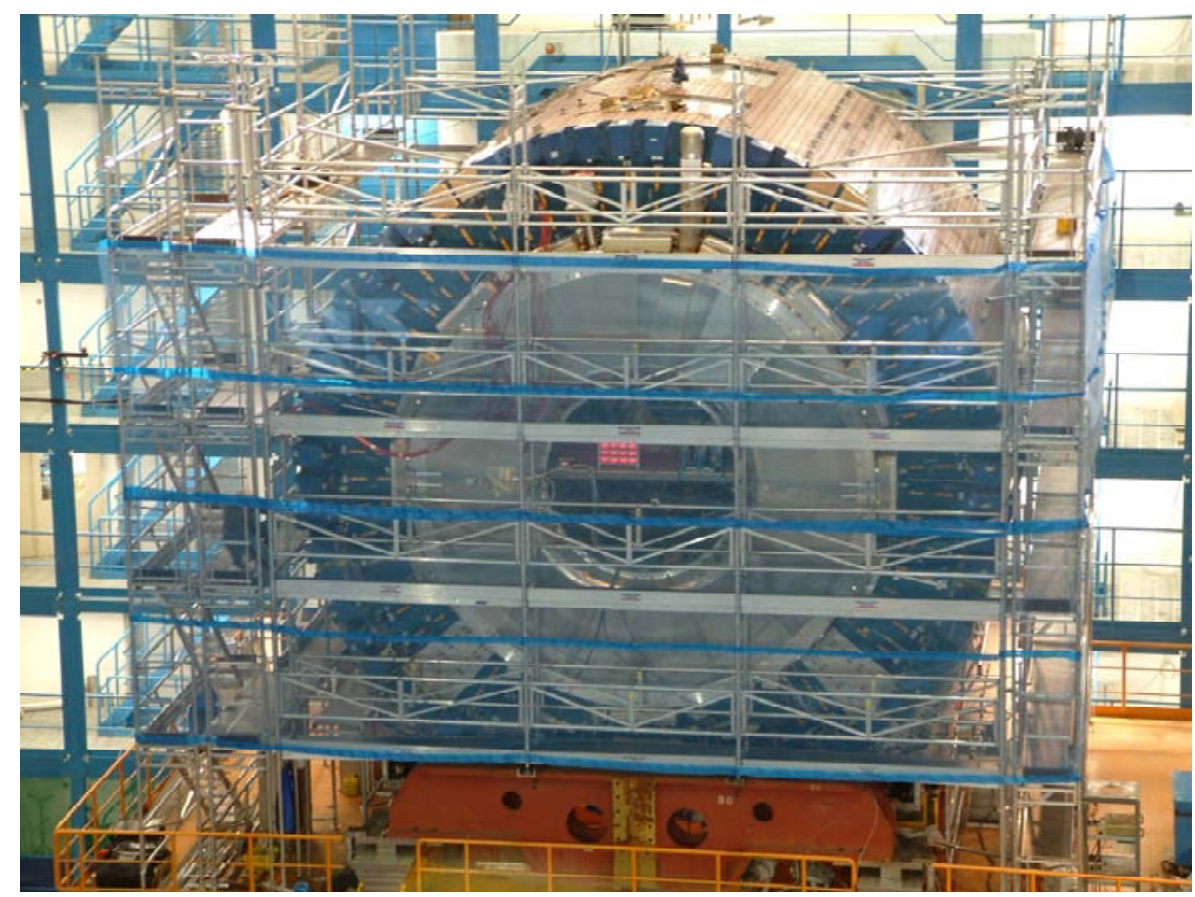

Figure 2. The Tile and Liquid Argon Barrel calorimeters assembled in the Atlas cavern. 
agreement between the horizontal deflections than the vertical deflections. It is felt that this is due to increased compression of the IR shims due the significantly increased front plate bearing force once the cryostat load is applied. These will be even more of an issue for the final assembly and in fact the final shim plan cannot be determined until after the cryostat has been loaded on the Extended Barrel.

An Argonne physicist led the group responsible for controlling the geometry of the barrel cylinder during the final assembly. Some shim adjustments (computed using the model that was developed during the surface assembly of the EBC and EBA) were made during the final assembly to bring the position of the top modules closer to the nominal position from that achieved during the surface assembly $(-9 \mathrm{~mm}$.) The result was that the last module was inserted in a gap which was no smaller than $-2 \mathrm{~mm}$ and there was no perceptible movement of the cylinder when the final module was inserted.

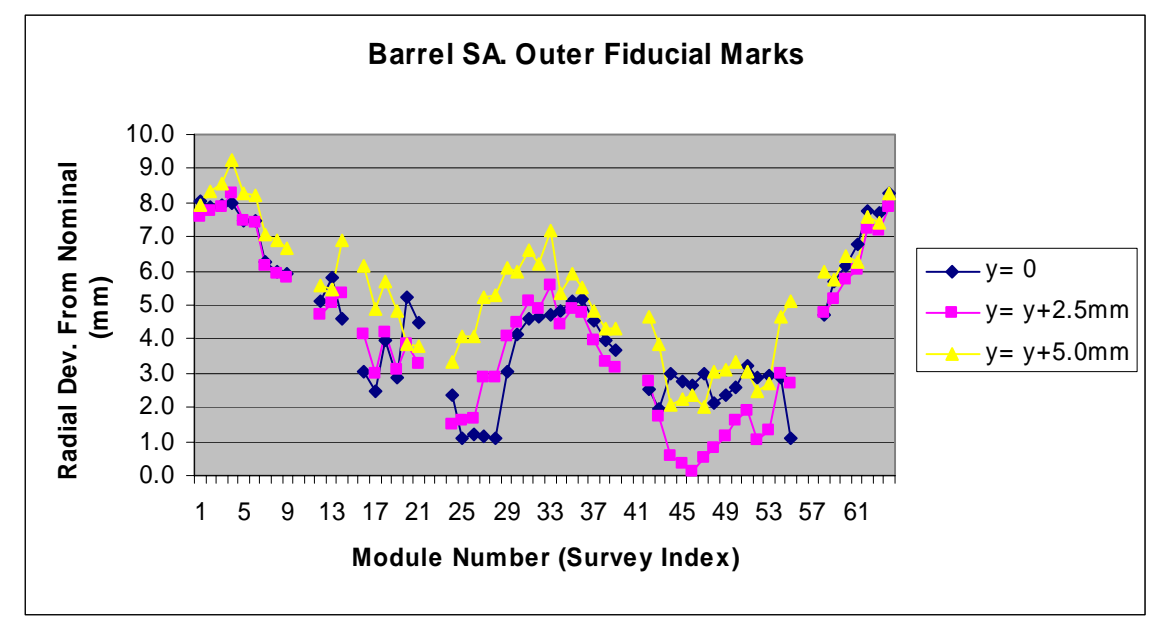

Figure 3. Radial envelope of the outer perimeter of the barrel cylinder compared to its nominal value (as a function of module number.)

The radial envelope of the outer perimeter of the barrel cylinder is shown in Fig. 3. The design specification is $+5,-10 \mathrm{~mm}$ and the cylinder is slightly outside this in the horizontal plane. However, there is an integration tolerance of $5 \mathrm{~mm}$ and no interference is expected with the muon system.

(J. Proudfoot)

\section{b) Installation of Services}

An Argonne physicist is coordinating the installation of services and he along with Argonne technicians visited CERN on several occasions in this period to develop and test installation procedures as well as to install services on the lower half of the barrel cylinder in the cavern. An example of the work carried out is shown in figure 4. The task 
is to work from a CAD layout to an actual realization of the services location and routing. Seemingly simply issues such as the width, depth and location of the cable trays require significant evaluation due to the tight integration tolerances between the tile calorimeter and the surrounding detector systems. Several poorly defined service attachments were worked out. An ANL technician assisted the CERN team in fabricating and installing the Azimuthal cable trays on the bottom half of the detector. In addition, the CANbus daisy chain cable attachment from finger-to-finger was worked out and certified by TC and the inner detector.

Modifications to the finger cables were made during this period. This included the addition of some sense lines for the power supplies and the addition of wiring harnesses to the existing Harting connector. The LV and ADC/HV CANbus daisy chains were completed in two sectors, and these were successfully certified with a microprocessor-based field tester.

Work for the LV cabling was done, with the fabrication and mounting of the Starboxes on the saddle box-beam by an ANL tech. LV cables were routed from the fingers to these Starboxes, and the lengths of the final (LV) cabling were determined so that immediate cable fabrication could occur.

At the end of the year, we were in a great position to finish the cabling immediately after the cooling pipes for the Inner Detector were completed.

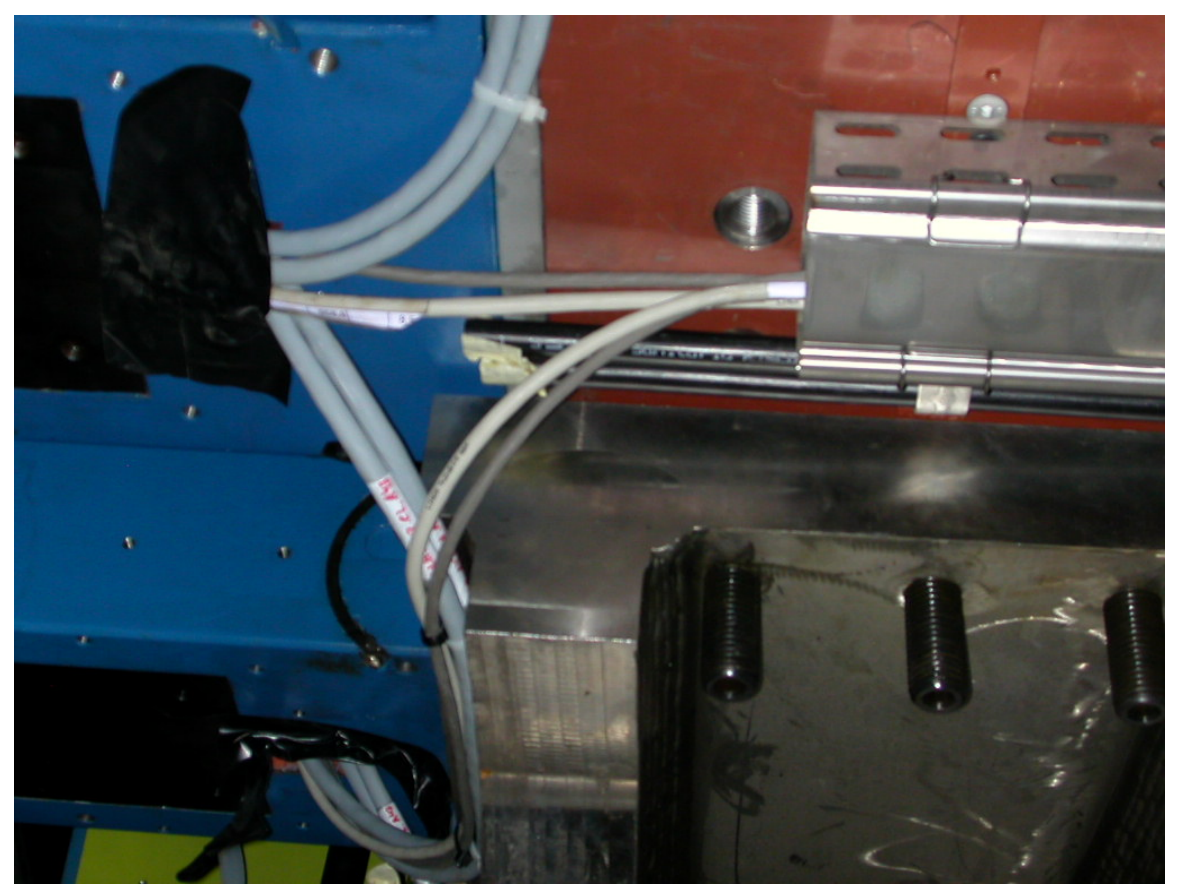

Figure 4. The final routing of the daisy chain and the LV cables in the difficult region around the saddles. 


\section{c) Calorimeter Commissioning}

An Argonne physicist is co-leading the planning and organization of work required to commission the tile calorimeter. ANL has undertaken the responsibility to lead the effort "at-detector". This includes

- Module Standalone Certification using MobiDAQ (a customized, portable, selfcontained DAQ system). Temporary cables and fibers the lengths of Extended Barrel ones were installed and routed to USA15 where MobiDAQ resides. The tests carried out include:

- Check for laser fiber integrity

- Check of correct operation of HV board

- Verify trigger signal pulse height

- Check of ADC card operation at $100 \mathrm{~Hz}$ through CANbus

- Obtain QC information on the modules and drawer electronics

- Cable and fiber specification, purchase and help develop the cable construction facilities.

- Implementation of the Rack Wizard to track cabling endpoints

- Design and implementation of user cabling labeling schemes

- Procurement and installation of equipment in USA15 racks

- Services Installation

- On detector services

- From USA15 with connector installation \& Checkout

- Barrel fully Connected to USA15 September 2005 following the move to $\mathrm{z}=0$

- Development of USA15 DAQ towards final implementation, including

- DAQ software, TTC, LVL1 I/F, Calibration, Monitoring, DVS

- DCS

- Multiple (8) drawer readout using MobiDAQ and eventually a final RODbased partition

(R. Stanek)

\section{d) Software Development for Tile Commissioning}

In order to test the Tile Cal drawer modules as they are installed in the calorimeter, a data acquisition system, called MobiDaq, based on the Rod Crate DAQ software was developed. The system, which reads out eight drawer modules, is built upon a VME crate with a Rod Crate Controller and four Rod Emulator boards, each of which reads two drawer modules. MobiDaq has the ability to collect the data on a notebook PC from all the drawers for sampling and monitoring of data from cosmic ray events, as well as charge injection and light sources. 
A software professional from Argonne is contributing to the development of this system. As a first task, a quick test of the basic functioning of the electronics running at $100 \mathrm{kHz}$ before a data taking run was implemented. As a longer term objective, associated with integration into the Atlas TDAQ system, a framework was developed for using the TDAQ Detector Verification System (DVS) that would set up the drawer electronics, perform simple checks of pedestal data and report results. The framework consists of applications based on two $\mathrm{C}++$ classes, one which configures the drawer electronics from the database and controls the triggering and another which represents the data fragments read from the drawers with methods that analyze the contents. Two specific methods were implemented, one which checks the events for consistent BCID values and another which calculates and checks the CRC values provided each digitizer chip. Some additions and modifications were also made to the schema, which describe the TileCal detector to make it useful to the test manager in the DVS. This work will continue in 2005 .

(J. Schlereth)

\section{e) Movement system tests at Argonne}

\section{TC Movement Hardware}

While the design and analysis of the movement hardware components were completed previously, the design of the extended barrel Z-bracket required modification based on the final hydraulic cylinder choice. The FEA analysis required to complete this design is illustrated in figure 5. Additionally, a spring roller mechanism was designed for supporting the Z-bracket.
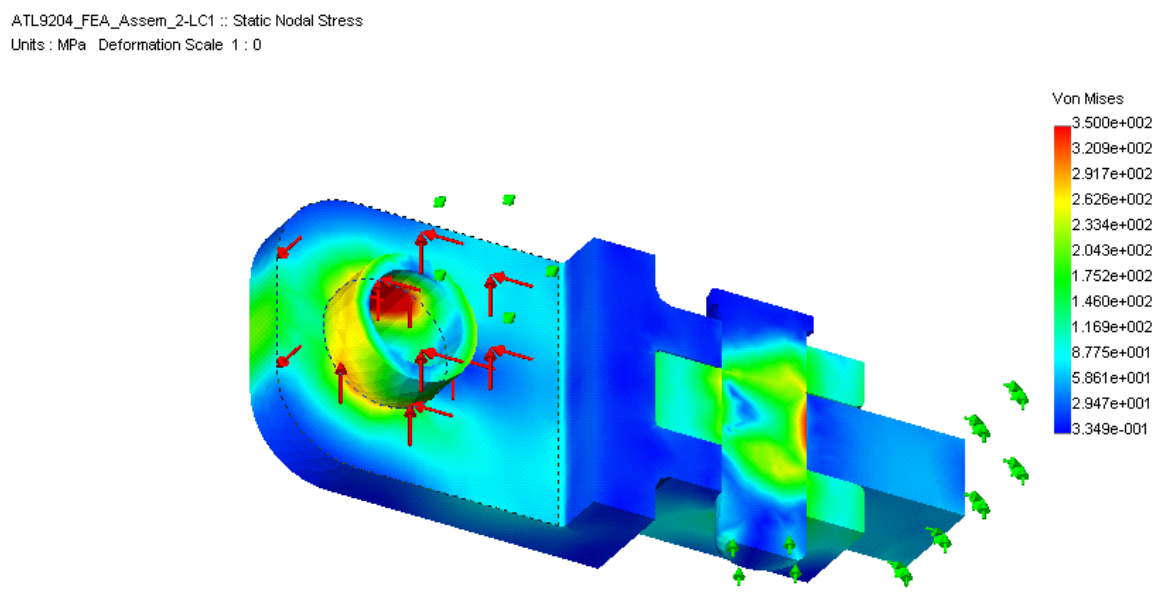

Figure 5. FEA Analysis of the modified extended barrel Z-bracket cylinder link connection after final cylinder dimensions were received. 


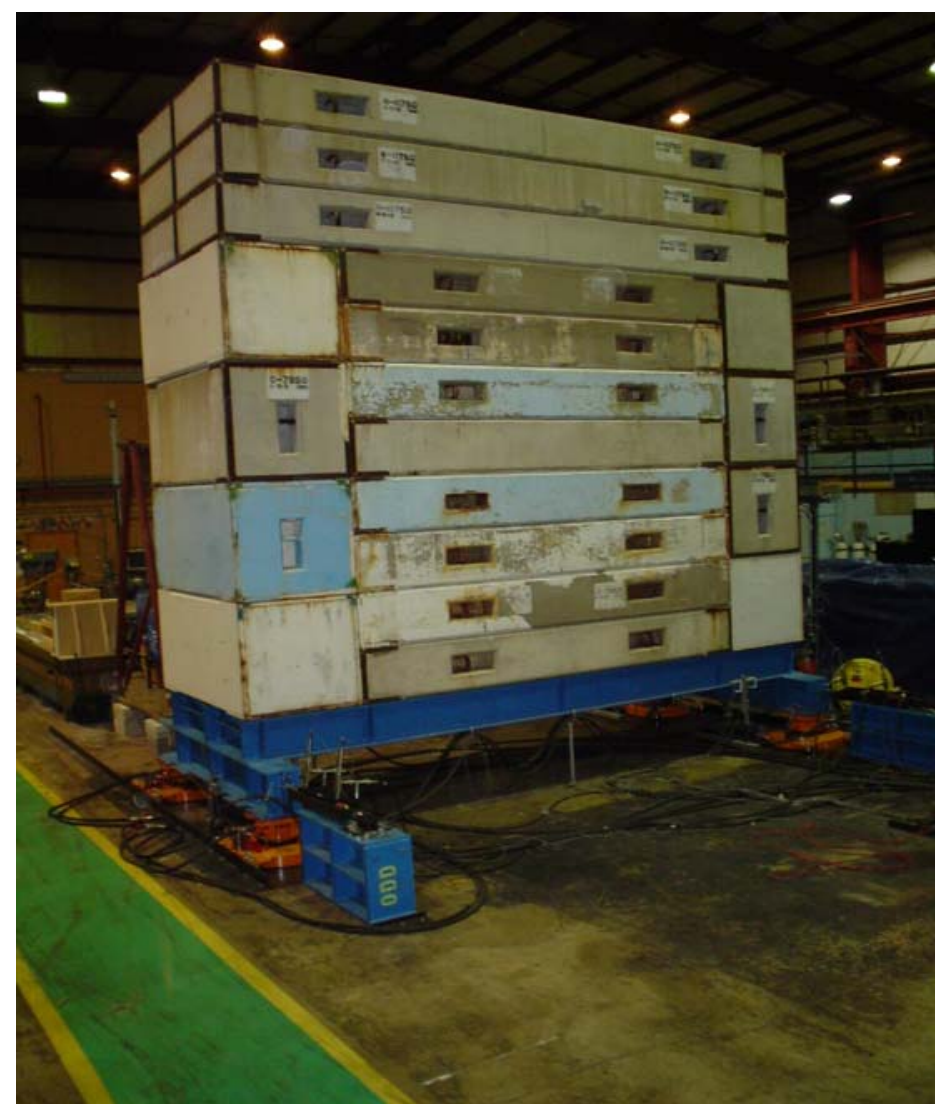

Figure 6. The airpad test set-up fully loaded with 120 tons.

\section{f) Movement Control System}

With the use of the airpad test set-up at ANL, the movement system made significant progress during the time period July through December 2004. In July, the first movement tests were run with the leveling system active with 66 tons loaded on the frame and then the load was increased to the full 120-ton load (figure 6.) Subsequently, the automatic leveling function was tested on the 120-ton stack for the first time and several problems were uncovered. Several tests were conducted with regard to solving these problems identifying limits on the hydraulic and pneumatic pressures during the tests. In addition, others tests were performed to improve the horizontal motion control and for understanding of the force measurement on the horizontal cylinders. This culminated in a successful movement of the 120 ton stack with automatic leveling and a better controlled horizontal movement speed. Over the entire time period, 34 trials were run to resolve minor problems, gain further system understanding, and provide verification for the performance. In October, the automatic raise and lower function 
which was successfully implemented. The results from one such test is shown in Fig. 7

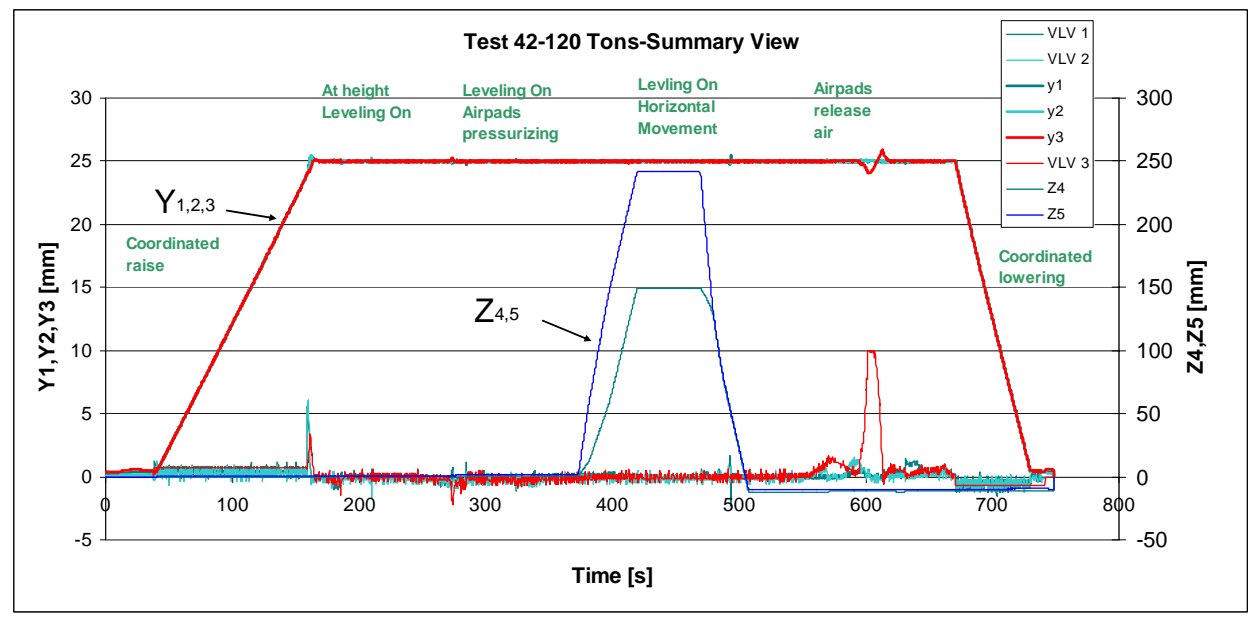

Figure 7. Summary view of movement data collected during trial run with 120 tons. The error in the height control (Y) is generally less than $0.1 \mathrm{~mm}$ with only a brief excursion during the airpad air release. At all times during the motion, the height error is less than $1 \mathrm{~mm}$.

At the end of this time period, much effort was put into preparations for the final system tests at ANL, in particular the simulation of the detector docking. This included the design and analysis of frame supports to act as the blocking jacks allowing removal of load from the airpads, conversion of the hydraulics to allow individual cylinder control, the addition of stops and shims for simulating the docking procedure, and preparation for the addition of the emergency air purge valve to the system (figure 8.) Tests of these parts of the system will begin in early 2005 .

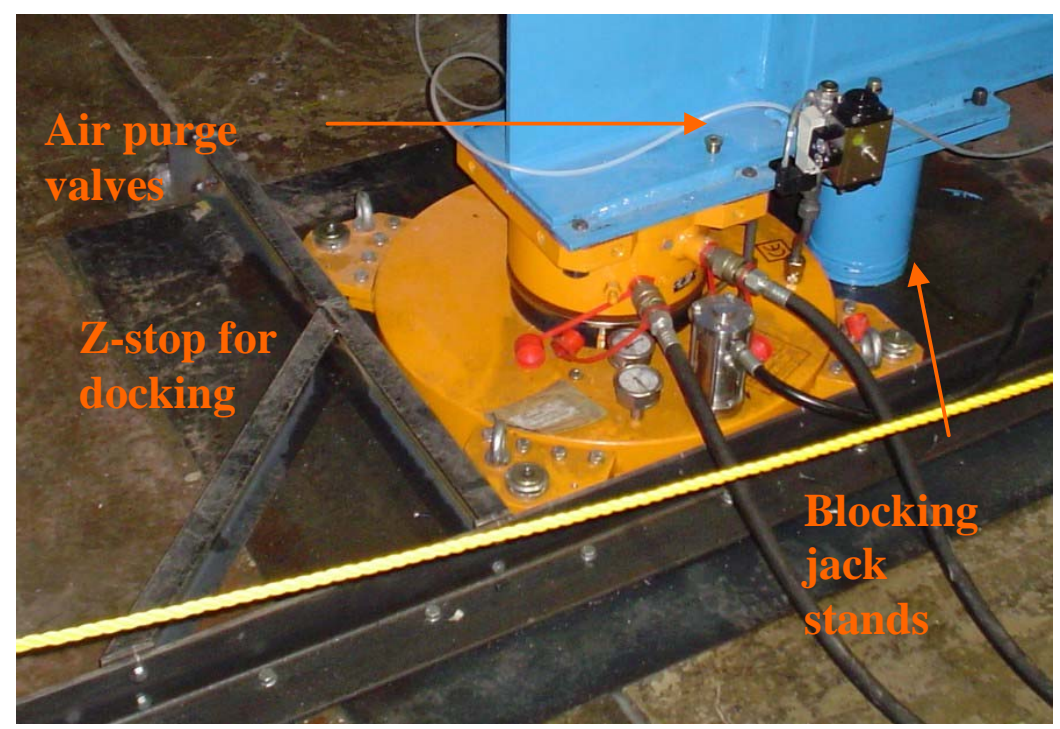

Figure 8. Components added in preparation for final docking and safety tests. 
At the same time, effort went into understanding and implementing the BCAM position monitoring system into the test set-up. These were attached to the system and a movement test was completed with a preliminary data acquisition routine storing data in the PC.

Significant effort went into planning for the final control system design and the barrel movement test planned for 2005. This included effort went into the collaborative evaluation of the hydraulic power unit bids with CERN which culminated in the awarding of the contract. With this, the process of converting the control system for this new power unit has begun with the solicitation of information and the selection of new electrical components as needed. Finally, detailed plans to begin complete the tests at ANL in January have been made in Process of being carried out.

(J. Grudzinski)

\section{g) Simulation Studies}

Good progress was made in the second half of 2004 to increase the participation of Argonne physicist in Atlas physics - which at this point in time clearly is a simulation exercise. An Argonne physicist is co-leading a larger physics group comprising physicists from several institutions in the Mid-West. The group has met three times in this period, with the latest meeting being held at ANL. The group has a web page with tutorials, pointers to datasets and other information. Members of the Argonne group have contributed to this by providing simple examples for running an analysis in ROOT, or in Athena (the Atlas software framework.) A key interest of the group at Argonne is setting the jet energy scale, using the fairly standard techniques developed at CDF. The probe processes themselves ( $\mathrm{Z}$, gamma plus jets, and two photon production) are also interesting standard model processes and their study provides an opportunity for the Division to have an early impact of Atlas physics. Unfortunately, the Atlas reconstruction code has been going through rapid development cycles. Therefore, large-scale reconstruction will not take place until the beginning of 2005. Once this occurs we expect to quickly produce meaningful physics plots and to begin a detailed evaluation of the performance of the detector using the results of full simulation.

(J. Proudfoot) 


\section{I.C. NEW PROJECTS AND DETECTOR DEVELOPMENT}

\section{I.C.1. Computational Projects}

\section{a) ATLAS Computing}

In this reporting period, the second ATLAS data challenge (DC2) began. The purpose of DC2 was to test the ATLAS computing model - utilizing worldwide computing resources to process the large quantities of data produced by ATLAS. Additionally, data from the combined test beam (CTB) was produced, processed and analyzed in this period. The combination of two major activities simultaneously provided ATLAS computing with their greatest challenge to date.

Within the international ATLAS organization, David Malon remained responsible for the multi-petabyte distributed event store, and for core data management and database software generally. He also served as a member of the ATLAS Computing Model Task Force, which successfully delivered a document describing an ATLAS computing model and its resource implications to LHC reviewers in December 2004. A highlight of this reporting period was organization and successful completion under David's coordination of a test of event data flow infrastructure from the ATLAS event filter through initial reconstruction, construction of analysis objects, streaming, and population of a cumulative event-level metadata system ("tag"database). This database "Tier 0 exercise", part of DC2, provided the only end-to-end test to date of the viability of the proposed ATLAS computing model.

During this period, ANL hired a new software professional, Jack Cranshaw. He was placed in charge of a group finishing a set of validation tests for the POOL software used by Athena that is needed for DC2. After completion, most of the tests have been put into the nightly validation suite. He developed the test of writing multiple file input/output and multiple streams and the test which loads all the POOL converters within the ATLAS release, which allows us to make a generic reading test to help detect data model (schema) changes. Other tests and utilities were developed by ANL's Kristo Karr, including a prototype algorithm that extracts event tag metadata from an AOD stream and stores it in a POOL attribute list object for subsequent creation of an event tag collection further downstream. Jack Cranshaw is now also serving as the secretary for the Database Best Practices Group. This group has begun reviewing components of the database, and the first review was chaired by Sasha Vaniachine.

ATLAS has formed a Distributed Database Services group, and ANL's Sasha Vaniachine was selected to lead it. The dramatic increase in ATLAS database services load needed to handle CTB and DC2 was handled thorough coordinated worldwide deployment of replica servers including a high-capacity server at the ATLAS Tier 1 center at BNL, that supported US ATLAS DC2 production on Grid3. This group also 
deployed database monitoring services that enabled identification of the potential bottlenecks (critical for planning of future ATLAS database services expansion) and release of ATLAS Database Client library with advanced capabilities necessary for ATLAS distributed database services operations. Additionally, an LHC-wide project on Distributed Deployment of Databases has been launched and Sasha Vaniachine serves as the representative for ATLAS.

Tom LeCompte continued as International ATLAS Planning Officer. The work plan and estimated completion date is updated monthly, and has led to adjustments in the scope so that ATLAS software is ready at LHC turn-on. The ATLAS Software and Computing Project is now fully integrated with the overall ATLAS planning tool (called PPT). Preparations have begun for a series of subsystem reviews to be held in early 2005. These reviews will focus on what was learned from DC2, and how to use the information gained in future activities.

(T. LeCompte)

\section{I.C.2. Detector Development for the Linear Collider}

In the second half of calendar year 2004 the Linear Collider Detector R\&D group pursued their efforts aiming at the development of a design of the hadron calorimeter for the Linear Collider detector. Progress was made on both the detector simulation and the hardware development front.

\section{a) Monte Carlo Simulation Studies}

Work on the ingredients to a complete Particle Flow Algorithm (PFA) continued. A track-calorimeter deposit matching routine based on the identification of the first interaction layer and using narrow cones to follow the hadronic showers from layer-tolayer was developed. Energy deposits in the calorimeter belonging to neutral hadrons were identified as the energy left over after subtraction of the charged particle energy and the energy belonging to electromagnetic showers as identified by the electromagnetic calorimeter. A photon finder was applied to identify the energy depositions in the calorimeter belonging to photons. Applying these algorithms to $Z^{0}$ decays at $\sqrt{s}_{s}=91$ $\mathrm{GeV}$, allowed to reconstruct the $\mathrm{Z}^{0}$ mass via its decay into a pair of jets.

This first version of a complete PFA was used to study the difference in response between calorimeters using Steel or Tungsten as absorber. The results indicated a small preference of Tungsten over Steel. The reconstructed mass of the $Z^{0}$ boson using jets measured with a Tungsten - scintillator calorimeter is shown in Figure 1. 


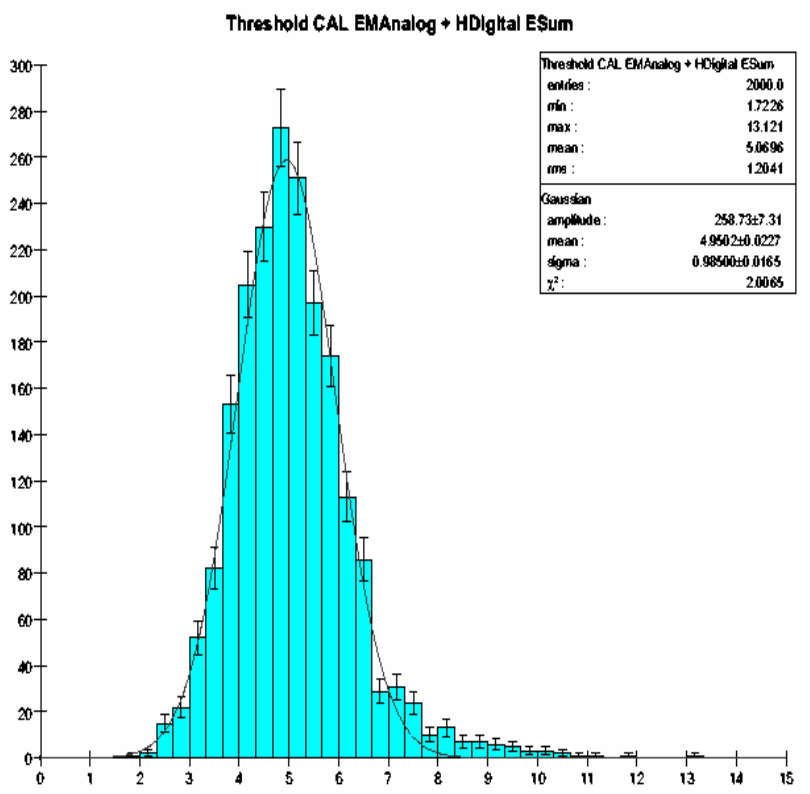

Figure 1. Reconstructed mass of the $\mathrm{Z}^{\circ}$ boson.

\section{b) Development of Resistive Plate Chambers as Active Medium of a Digital Hadron Calorimeter}

In the second half of 2004 the group continued its vigorous R\&D program to develop Resistive Plate Chambers as active medium of a digital hadron calorimeter. In the following we briefly summarize the major highlights of the project:

\section{1) Measurements of the Rate Capability}

The rate capability of the chambers was determined using a radioactive source at varying distance from the chamber to provide the rates and cosmic rays to determine the MIP detection efficiency. Figure 2 shows the results obtained with a chamber based on an exotic design. Due to the special geometrical arrangement of these measurements, the purity of the cosmic ray triggers is not $100 \%$, leading to a maximum apparent efficiency of $92 \%$. The efficiency is seen to remain at the maximum level for rates up to $50 \mathrm{~Hz} / \mathrm{cm}^{2}$. This measured rate capability is to be considered only as a lower limit, since the rate measurement itself is affected by the inefficiency of the chamber at higher rates. Chambers based on other designs were tested and performed very similarly. 


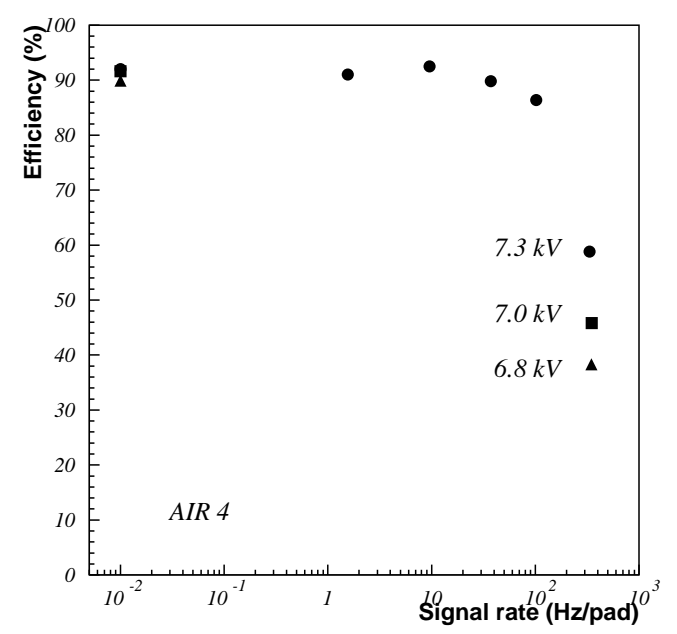

Figure 2. Measured MIP detection efficiency versus rate for an RPC based on our default chamber design.

\section{2) $\quad 1 \mathrm{~m}^{3}$ Prototype Section}

The goal of the present effort is to build a $1 \mathrm{~m}^{3}$ prototype section to a) test the technological implementation of RPCs in a calorimeter and b) to measure hadronic jets with unprecedented spatial resolution. The latter is necessary to fine tune the existing models of hadronic showers to be used in the overall design of a detector for the international linear collider. The section will consist of 40 layers each with an area of 1 $\mathrm{m}^{2}$. The active medium will be interleaved with $20 \mathrm{~mm}$ steel plates serving as absorber. The designs of the mechanical structure and of the test beam table are being developed at DESY and are almost complete.

\section{3) Conceptual design of the readout system for the $1 \mathbf{~ m}^{3}$ prototype section}

We developed a conceptual design of the readout system for the $1 \mathrm{~m}^{3}$ prototype section. With 40 layers of active medium, each with an area of $1 \mathrm{~m}^{2}$, and $1 \mathrm{~cm}^{2}$ lateral segmentation, the prototype section will feature of the order of 400,000 readout channels. This large number of channels demands a novel approach to the electronic readout system. Our conceptual design consists of four parts: a front-end ASIC located on the readout boards of the RPCs, a data concentrator located on the side of the prototype section, a VME based data collection system and a timing and trigger system. See Figure 3 for more details. The specification of each part of the system is in progress. 


\section{4) Design of the Front-end ASIC}

The design of the front-end ASIC progressed well. The digital part of the circuitry is completed. The design of the remaining analog part, containing the input circuitry, the preamplifiers, shapers and the discriminators, was delayed due to a conflict with other design efforts. The schematic of the chip layout is shown in Figure 4.

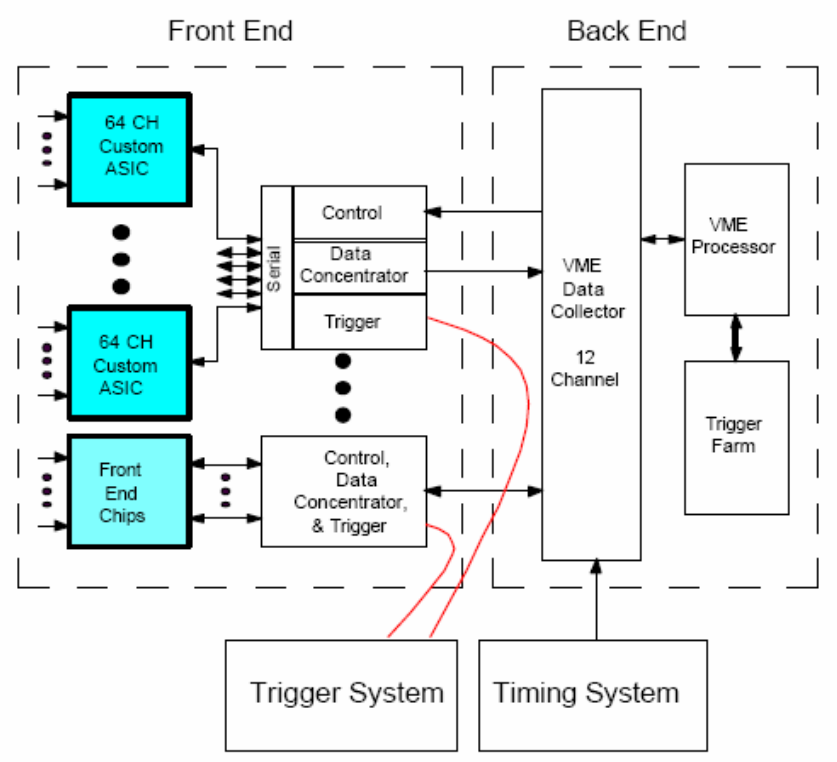

Figure 3. Schematic design of the electronic readout system for the $1 \mathrm{~m}^{3}$ prototype section.

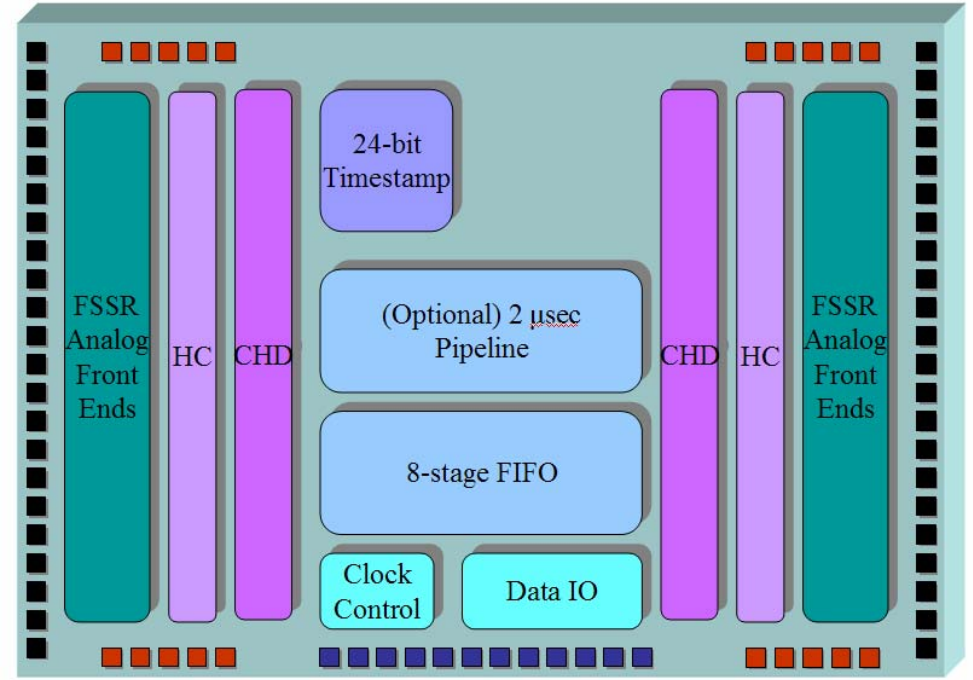

Figure 4. Schematic layout of the front-end ASIC being designed at Fermilab. 


\section{5) Collaboration Building}

To carry through the construction of the $1 \mathrm{~m}^{3}$ prototype section and to increase its likelihood of being funded, considerable effort was invested in building a collaboration involving outside institutes. Table I shows the members of the collaboration and the preliminary assignment of the various subtasks to each institute.

\begin{tabular}{|c|c|}
\hline \multicolumn{2}{|r|}{ Table I } \\
\hline Institutes & Subtasks \\
\hline Argonne & $\begin{array}{l}\text { Mechanical construction of RPCs } \\
\text { Overall design of the electronic readout system } \\
\text { Design and testing of the test front-end readout boards }\end{array}$ \\
\hline Boston University & Design and prototyping of the data collector \\
\hline University of Chicago & Design and prototyping of the data concentrator \\
\hline DESY & Design and construction of the mechanical support \\
\hline Fermilab & $\begin{array}{l}\text { Design and testing of the front-end ASIC } \\
\text { Design and prototyping of the front-end readout boards }\end{array}$ \\
\hline Iowa & $\begin{array}{l}\text { Design and prototyping of the HV system } \\
\text { Design and prototyping of the gas distribution system }\end{array}$ \\
\hline $\begin{array}{l}\text { University of Texas at } \\
\text { Arlington }\end{array}$ & Design and prototyping of the trigger and timing system \\
\hline University of Washington & Development of the data acquisition system \\
\hline
\end{tabular}

\section{6) Preparations for the test beam}

In order to prepare for the test beam at Fermilab, we drafted a detailed Memorandum of Understanding (MoU) between the groups participating in the development of calorimeters for the Linear Collider and Fermilab. The MoU spells out the specific needs for the various tests currently being prepared. In particular, the MoU requests three major upgrades to the existing MTB6 beam line:

- Improvement of the duty factor from $1 \%$ to at least $5 \%$

- Extension of the range of pions to low momenta, as low as $1 \mathrm{GeV} / \mathrm{c}$

- Extension of the range of electrons to low momenta, as low as $1 \mathrm{GeV} / \mathrm{c}$.

(J. Repond) 


\section{I.C.3. Electronics Support Group}

ATLAS: We have major responsibilities in the development of electronics for the Level 2 Trigger of the ATLAS Detector at CERN. Working with colleagues from Michigan State University, we are responsible for the development of two primary components of the Level 2 Trigger System, called the Trigger Supervisor, and the Region of Interest (ROI) Builder. These are major projects for our group, and they are key components of this system.

The Trigger Supervisor controls all activity in the Level 2 System, including the allocation of Level 2 Processors and the passing of selected events to the Event Builder. This was originally designed at Argonne as custom hardware, and was capable of operating with a variety of computers and bus interfaces. The initial work was performed in the mid-1990's, and Argonne provided hardware and software support for many of the early tests to study system architecture. The current plan for implementation of the Trigger Supervisor in the final system is to use commercial processors, since the system architecture is now finalized. Argonne will provide software support for the final implementation.

The ROI Builder is the interface between the first level trigger and the second level trigger. When an event occurs in the detector, information is sent from the frontend electronics to the Level 1 Trigger. The Level 1 Trigger collects event fragments from the front-end electronics over the entire detector, and stores them in a Readout Buffer. It evaluates the data, and identifies regions of the detector that could have an interesting event. The Level 1 Trigger then sends a list of addresses called pointers to the ROI Builder, identifying where the event data from that particular "Region of Interest," can be found. The ROI Builder collects all the pointers for the event, and "builds" the event using the pointer list. It then sends the result to the Trigger Supervisor for distribution to Level 2 processors. The selected Level 2 Processor then executes algorithms using the pointers, and can request information to be sent from the Readout Buffers as needed. The result is a Level 2 Trigger Decision for that event. If the event is accepted, then the entire event is read from the Level 1 Readout Buffers for further processing by higher levels of the trigger system.

The ROI Builder resides in a VME Crate that is part of the Level 2 Trigger System. It consists of three types of boards. The first is called the Input Card, which contains circuitry that receives and buffers input data streams from the Level 1 Trigger System. Each card can receive three data streams from the Level 1 Trigger, one stream from each of the major subsystems in the ATLAS detector. The trigger system can have up to four such cards in the system, for a total of 12 input streams. The data that is collected on the Input Cards for a given event is sent to another card that resides in the crate, called the ROI Processor. This is a sophisticated VME data processor, which uses high-speed, high-density Field Programmable Gate Arrays (FPGAs) to implement the 
functionality. Each FPGA contains over 50,000 gates, and there are 24 of these devices on the board. There can be up to four ROI Processors in the system. Each operates on separate events at a given time. The data transfer between the Input Cards and the ROI Processors is accomplished using a custom backplane in the crate. All three components of the system were designed at Argonne.

In earlier reporting periods, we built the first version of ROI Builder. It has undergone extensive testing at Argonne and at CERN. In 2002, an internal ATLAS review committee reviewed the project, and certain improvements were recommended associated with error reporting and data slow control. In 2003, we created a new design document that incorporated the recommendations. We then proceeded with the design of a new version of the Input Card and the ROI Processor. In the first half of 2004, new versions were fabricated, assembled, and tested in the lab. These tests used only a simple custom backplane, which accommodated one Input Card and one ROI Processor. The system performed very well in tests, and met all of the recommendations made by the review committee. In this reporting period, the system was sent to CERN for tests in the ATLAS testbeam. Several bugs were uncovered and fixed. In this period, work also proceeded on the design of the full version of the custom backplane. In the course of this work, it was found to be necessary to modify the data drivers on the Input Card, to ameliorate capacitive loading problems. Both the custom backplane and the modified Input Card are currently being fabricated. Testing will be performed at Argonne in the early part of 2005. We plan to send the full system to CERN for testing late spring of 2005. We intend to perform a full system test at that time, which will include four Input Cards and four ROI Processors. This will be the final benchmark for the system prior to installation in the experiment.

MINOS: Argonne physicists and engineers have been involved with the development and construction of MINOS, the Neutrino Oscillation Experiment at Fermilab. The experiment is designed to study the physics of neutrinos by measuring their properties in two locations, spaced over a large distance. A neutrino beam is generated at Fermilab. It is passed through a detector at Fermilab called the Near Detector, where the composition and other properties are measured. The beam leaves the Near detector and is directed through the earth toward the Soudan mine in northern Minnesota, where a second detector called the Far Detector has been built. By measuring the properties of the neutrino beam at the two locations and comparing the results, important aspects of the physics of neutrinos such as mass and CP violation can be measured.

Argonne had major responsibilities for the development of electronics for the design, development, and production of electronics Near Detector. One member of our group was the Level 3 Manager for the Near Detector Electronics. In addition, we were responsible for designing and building many of the electronic components for the Near 
Detector, along with Fermilab. This has been a major project for our group over the last several years.

The beam at Fermilab will deliver protons to a target over a $10 \mu \mathrm{Sec}$ period, called a spill. The electronics must measure all interactions that occur in the detector during this time, with a timing resolution of $\sim 20 \mathrm{nSec}$. The detector is composed of steel and scintillator, and is instrumented with multi-anode photo-multiplier tubes. The readout system is based around a custom integrated circuit developed at Fermilab called the QIE. The QIEs receive charge pulses from photo-multiplier tubes, and digitize continuously at $53 \mathrm{MHz}$. The operations are pipelined so that there is no deadtime due to digitization. The digitized data is stored in a local memory during the entire period of the beam spill. The data is sent from the local memory to a read-out board after the spill is over. In between spills, the electronics records data from cosmic rays.

The QIEs and associated circuitry are built on small daughter boards called MENU Modules, which resemble memory SIMMs. The boards contain a high density of surface mount parts. The MENU Modules plug in to a motherboard called the MINDER Module. The MINDERs reside in front end crates called MINDER Crates, which are a semi-custom design. There is a crate controller in the MINDER Crates called the KEEPER, which controls all activity in the crate. When data is acquired, it is stored on the MENU Modules. After data is acquired, the MINDER then initiates a readout operation, where the data is sent from the MENUs to a VME readout board, called the MASTER Module. The MASTER resides in a 9U VME crate located some distance away from the MINDER Crates. All of the board designs contain a high level of programmable logic to do the complex processing of data and control of operations.

The chip design, and the development of the QIE daughter board, were the responsibilities of Fermilab. Argonne was responsible for the design the MASTER Module, the MINDER Module, the KEEPER, the MINDER Crate, signal and data cables, and AUX cards for receiving signals in through the back of the VME Crates. Argonne was also responsible for infrastructure, crates, and power supplies. As part of the Level 3 Management, Argonne had responsibility for the overall design and performance of the entire system.

Throughout the course of this project over the last several years, Argonne personnel lead the design of the architecture of the system. The functionality of the QIE was specified, prototype chips were designed, built and tested through Fermilab, and 20,000 chips were produced. We designed and built two rounds of prototypes of all system components, including the MENU, the MINDER, the KEEPER, and the MASTER. We built and constructed a "Vertical Slice" test stand at Argonne, to test the full functionality of the system. This test stand was also crucial in the development of data acquisition and calibration software, which was also lead by Argonne personnel. 
We also participated in two running periods in a test beam at CERN called CALDET, which used the new electronics. Argonne personnel were actively involved in the installation and commissioning of electronics for these test runs, including support for the data acquisition software.

In 2003, we completed all system tests to verify that the performance of the system met specifications, and began final production of all system components. Argonne was responsible for the production of 100 MASTERs, 600 MINDERs, and 60 KEEPERs. We used outside vendors for the printed circuit board fabrication and board assembly. The checkout work was performed Argonne staff technicians. The production and checkout of all components were completed by the summer of 2004 . In this reporting period, we assisted with the installation, debugging, and commissioning of electronics on the detector. The experiment is scheduled to begin taking data in early 2005. We will provide assistance and support of the electronics in preparation for the beginning of the experiment, and throughout the running period.

Linear Collider Hadron Calorimeter: A new accelerator called the Linear Collider is still in the conceptual stage. However, work is currently in progress throughout the world to develop new detectors for this machine. Argonne physicists have been leading the development of a new hadron detector, which would be used in the central calorimeter of this new experimental facility. The current work is on developing a new detector made of Resistive Plate Chambers (RPCs). The RPC design for this detector uses fine-grained cells, having dimensions $1 \mathrm{~cm}$ by $1 \mathrm{~cm}$, and many independent sampling layers. These detector elements would be read out using amplifier/shaper/discriminators (ASDs), essentially a 1-bit ADC. The nature of the data acquisition is to capture images of the hadronic interactions in the detector as a function of time, where the images are composed of "pixels" that are either on or off. Events are reconstructed using a technique known as "Particle Flow Algorithms," which is a new concept that uses tracking information as part of the reconstruction of energy deposition in the calorimeter. The traditional approach to hadronic calorimetry is to construct detectors having projective geometry, where many sampling layers are summed together to form a relatively small number of readout channels. This new approach trades the read-out of a small number channels having large dynamic range, 12 bits or more, for reading out a large number of channels with only 1 bit of dynamic range. A goal in the project is to develop very inexpensive electronics, since the channel count will be high.

In 2002-2003, small prototype detectors were built at Argonne. They were tested in a cosmic ray hodoscope that was constructed at Argonne. We provided a data acquisition system and read-out electronics for making measurements on the prototype detectors. The test results have been very encouraging. Work was also begun to design a larger intermediate detector, This detector would be a cubic meter of RPCs sandwiched with steel plates, having 40 sampling layers, and would have $400 \mathrm{~K}$ readout channels. 
Due to the large number of readout channels and the low dynamic range needed, it is envisioned that the front-end electronics would incorporate a custom integrated circuit. A goal is to construct this detector, complete with electronics, and test it in a test beam at Fermilab in 2006 . The development of electronics for the intermediate detector is a collaborative effort between Argonne, Fermilab, Boston University, and the University of Chicago, with Argonne having the leading role in the system engineering.

In the last reporting period, we developed a conceptual design for the read-out system. In order to keep costs down, we intend to use a custom ASIC in the front-end electronics. We developed a conceptual design for this device, and wrote a complete specification. We formed an alliance with the Fermilab ASIC Design Group, and work began to realize the design. In this period, work continued on the design of the chip, but was delayed due to competing priorities. We expect that the design of the prototype chip will be completed in the spring of 2005, with a small number of chips back from fabrication by the summer. We plan to test the devices at Argonne. In addition, we are working on the design and specification of the other parts of the prototype read-out system. We have formed a core design group, and are working on detailed specifications of the system components. We expect that the design of prototypes will begin in early 2005 , with the goal of performing small-scale tests of the system by the fall.

Veritas: Physicists at Argonne have joined Veritas, which is an experiment designed to study high-energy cosmic rays using an array of ground-based telescopes. When cosmic rays enter the earth's atmosphere, they interact with it, which results in the emission of light pulses. The telescopes are used to capture and digitize the light radiated in the night sky, and to reconstruct the energy and direction of the incident particles. There are several important HEP science topics associated with the study of cosmic rays, including dark energy, neutrilinos, and quantum gravity, and the area of study has attracted many HEP scientists worldwide. The nature of the read-out systems for these telescopes is approaching the sophistication and complexity of a HEP experiment. These two aspects make Veritas a natural fit in both the science and the electronics development for the division.

The Veritas experiment is currently being built in Arizona, having an array of four telescopes. We are working with the University of Chicago to propose a new photodetector and high-speed electronics for the next phase of the project. This would be an upgrade, which would add three more telescopes to the array, and would also augment and enhance the capability of the current instrumentation. The new system would have $\sim 10,000$ channels of high-speed, high precision charge amplifiers in the final configuration. The Argonne group received a grant from the lab director to participate in this joint project between the University of Chicago and Argonne, which includes electronics development. 
In the previous reporting period, the Argonne group began an R\&D program involved with making measurements on multi-anode photo-detectors (MAPMTs.) The use of this type of photo-detector would be a new approach in this field, and would represent a significant cost saving if used in the upgrade to the experiment. To support the study of MAPMTs, we constructed a teststand and data acquisition system, and provided read-out software. The system is capable of injecting light in a precision way into a selected pixel of the photo-detector, and to measure the response of the MAPMT. The important performance parameters include sensitivity, noise, and linearity, with and without the presence of background light that would occur with night sky conditions. In this reporting period, this work continued. We provided support and assistance in making measurements on several different MAPMTs. We also assisted in making direct measurements of the night sky background, which is important in understanding the performance of the photo-detectors and the electronics.

A second goal for this project is to build a small telescope on-site at Argonne that would incorporate an array of MAPMTs. The instrument would be used to make measurements on the night sky as a proof of principle for the use of MAPMTs. In the previous reporting period, we began constructing a data acquisition system capable of reading out $\sim 250$ channels using high-speed electronics. In this reporting period, progress has been made in the development of the data acquisition software. The Argonne Veritas group purchased a trailer to serve as the electronics hutch for the instrument, and we assisted in the specification of the infrastructure needed to support the electronics and read-out system. Work will continue on the development of this instrument in 2005, with the goal of having it functional around the summer. Following on this work, we intend to make a proposal to build the electronics for the upgrade to the telescope, which would be presented in late 2005 or early 2006.

(G. Drake)

\section{I.C.4. Experiments to Measure the $\theta_{13}$ Neutrino Mass-mixing Parameter}

\section{a) $\quad \mathrm{NO} v \mathrm{~A}$ (NuMI Off-axis $v_{e}$ Appearance) Experiment}

Argonne physicists and engineers have been working on the design of a nextgeneration long-baseline experiment in the NuMI neutrino beam since 2002. The experiment takes advantage of the narrow energy spectrum of neutrinos produced a few degrees away from the beam axis, which enables a high sensitivity search for $v_{\mu} \rightarrow v_{\mathrm{e}}$ oscillations. The observation of $v_{\mu} \rightarrow v_{\mathrm{e}}$ allows a measurement of the $\theta_{13}$ neutrino-mass mixing parameter. The energy spectrum depends on the off-axis angle, which would be chosen to give a peak energy close to the first maximum of the oscillation probability. The reduced flux of high-energy neutrinos in the off-axis beam significantly lowers the 
background from neutral current events. Measurement of a nonzero value of $\theta_{13}$ could eventually lead to the construction of a second off-axis detector at a location where the peak energy was close to second maximum of the oscillation probability. Measurements at the two locations could resolve the neutrino "mass hierarchy" problem and measure the $\mathrm{CP}$ violation parameters. If the NuMI beam intensity can be increased sufficiently and the neutrino-mass mixing parameter is large enough, it is even possible that the NOvA experiment itself could see the first evidence for $\mathrm{CP}$ violation

In March 2004, the NOvA Collaboration, including Argonne and 33 other institutions, submitted a formal proposal (P-929) to Fermilab for the off-axis experiment. The Collaboration later submitted three Appendices to the proposal, which was then discussed at the June Physics Advisory Committee (PAC) meeting. Argonne physicists and engineers played major roles in writing the proposal and producing engineering designs and cost estimates. The NOvA proposal requests Stage 1 approval to build a 50,000-ton detector to search for $v_{\mu} \rightarrow v_{\mathrm{e}}$ oscillations, $12 \mathrm{mrad}$ off the axis of the NuMI beamline in Minnesota or Canada. Although the PAC did not recommend approval for NOvA, it did urge Fermilab to put the experiment on a "fast track" and to provide the R\&D funds needed to finalize the detector technology choice.

Before the proposal was written, the Argonne group had worked with Fermilab collaborators on a detector design using resistive plate chambers (RPCs). However, we have now decided to stop work on RPCs in favor of the liquid scintillator "baseline" technology chosen for the proposal. The baseline detector has planes of horizontal and vertical liquid scintillator strips (liquid-filled tubes in PVC extrusions, read out with wavelength-shifting fibers) interleaved with wood (particleboard) absorber material. The design of the liquid-scintillator design has continued to evolve since the proposal was submitted. The Argonne group, together with collaborators from Fermilab, Minnesota and Indiana devoted most of 2004 to development of a "totally active" detector design, which has alternating "walls" of horizontal and vertical PVC tubes that are filled with liquid scintillator and read out with wavelength-shifting fibers and avalanche photodiodes. This design has higher detection efficiency and background rejection for $v_{\mathrm{e}}$ events, allowing the detector mass to be reduced by a factor of two (to 25,000 tons) with no loss of event rate. It seems likely that advanced simulations, with detailed event reconstruction software, will show this totally active design to have even higher efficiency and background rejection.

During the second half of 2004, Argonne physicists and engineers devoted a substantial effort to studying mechanical engineering issues associated with the totally active design. These include handling, machining and bonding of the 53-foot long PVC extrusions, in addition to structural engineering considerations. The most fundamental issue, which was still under study by Argonne and Fermilab engineers at the end of the year, is the structural stability of the very large structure ( $53 \mathrm{ft}$ by $53 \mathrm{ft}$ by $350 \mathrm{ft}$ long), built entirely of thin-walled (2- to 3-mm thick), liquid-filled PVC extrusions. The 
Argonne mechanical support group has identified adhesives that are suitable for bonding end-caps to PVC extrusions and others that are appropriate for bonding $53 \mathrm{ft}$ by $53 \mathrm{ft}$ walls of horizontal and vertical tubes to each other.

The group has conducted many mechanical tests on small prototypes using commercially available, 32-tube-wide extrusions that have dimensions similar to those proposed for the detector. The results of pressure tests on individual tubes and of load tests of small prototype multi-wall structures are compared to finite-element analysis models, which can then be extrapolated to much larger structures that are needed for the NOvA experiment. Figure 4 shows a three-plane test assembly in the load-test fixture in Building 366. Detector assembly techniques are being tested on a 4-plane, $27 \mathrm{ft}$ high by $15 \mathrm{ft}$ wide "half-scale" prototype, which will be assembled in Building 366 in early 2005.

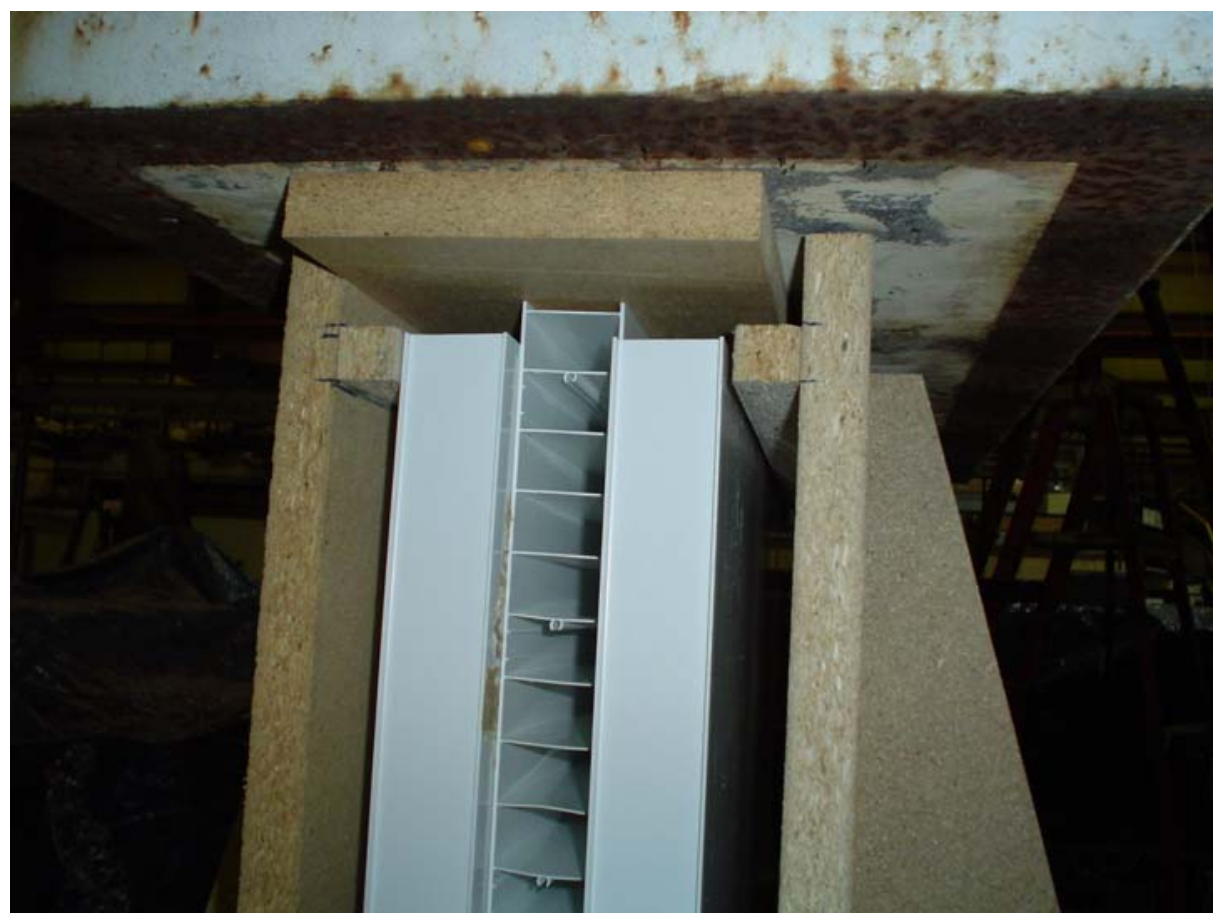

Figure 4. Load-test fixture for a three-plane assembly of NOvA extrusions. The two outer planes have vertical tubes. The load is applied to the center plane of horizontal tubes to test the strength of the adhesive bond between the planes.

Figure 5 shows a test assembly of two planes of this structure. The Argonne group has also begun a study of techniques for filling the empty tubes of the partially assembled detector with liquid scintillator. The detector must be continuously filled with liquid at an average rate of 15 gallons per minute during the two-year assembly period. At the end of December, the Argonne group had started studies of air-bubble accumulation in horizontal tubes and of the mechanical properties of PVC with different levels of $\mathrm{TiO}_{2}$, which must have a concentration of about $15 \%$ to achieve maximum reflectivity. Commercial extrusions typically have $\mathrm{TiO}_{2}$ concentrations around $10 \%$. 


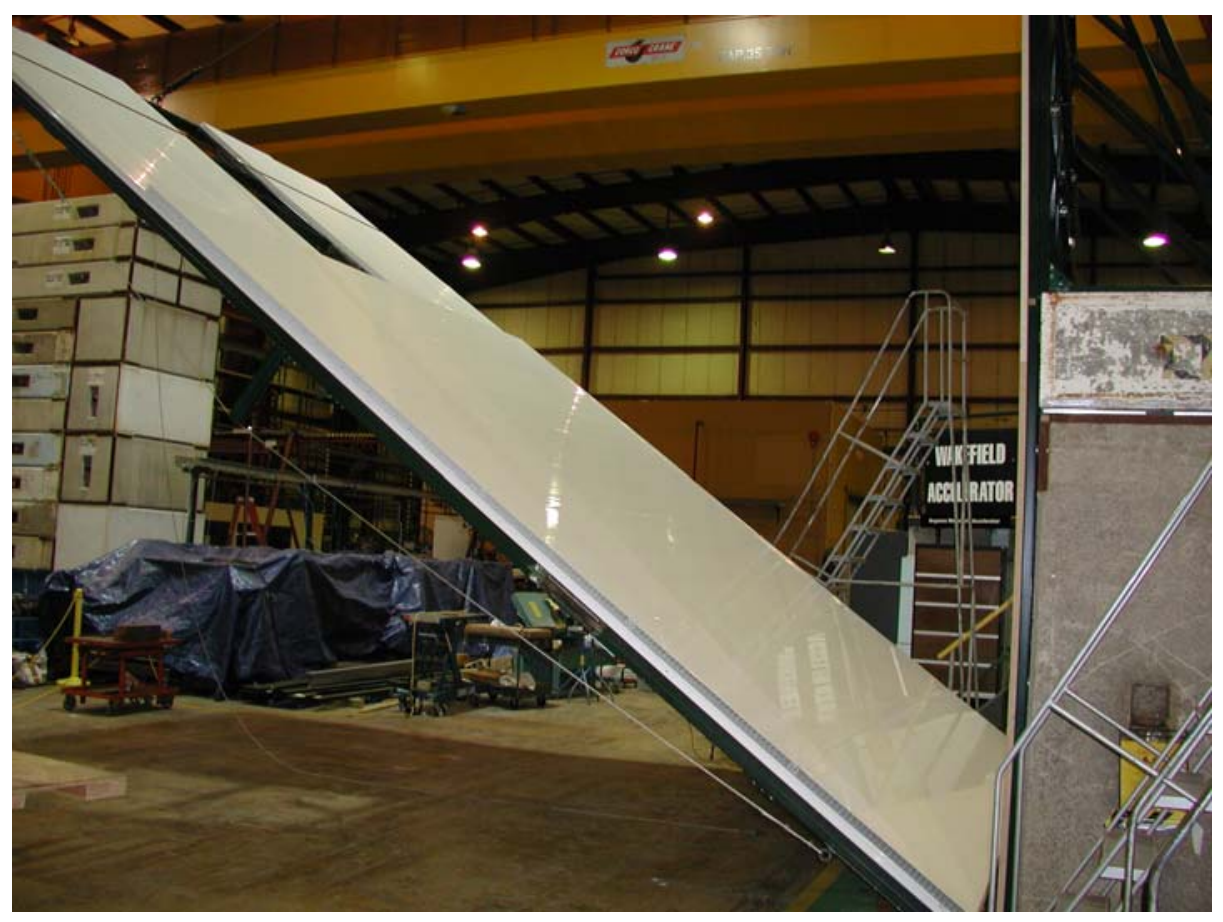

Figure 5. NOvA half-scale prototype assembly in Bldg 366 . The $27-\mathrm{ft}$ high by $15-\mathrm{ft}$ wide halfscale prototype of the NOvA detector is being built at Argonne to test structural properties and assembly techniques. Shown here is a trial of the assembly procedure in which single planes of horizontal and vertical PVC extrusions are placed on a frame and raised against a vertical "bookend" structure. For this test the extrusions were set in place on the frame but were not yet glued together.

In parallel with these studies of the structural properties of commercially available PVC extrusions, the collaboration has been working with an outside vendor to produce extrusions that have the dimensions needed for the NOvA detector. Following many trial extrusions, the vendor began a production run at the end of December. Figure 6 is a photograph of some of the extrusions produced during this run. Each extrusion has only three cells (tubes), instead of the thirty cells that will be used for the actual detector, in order to reduce the cost of the extrusion die. 


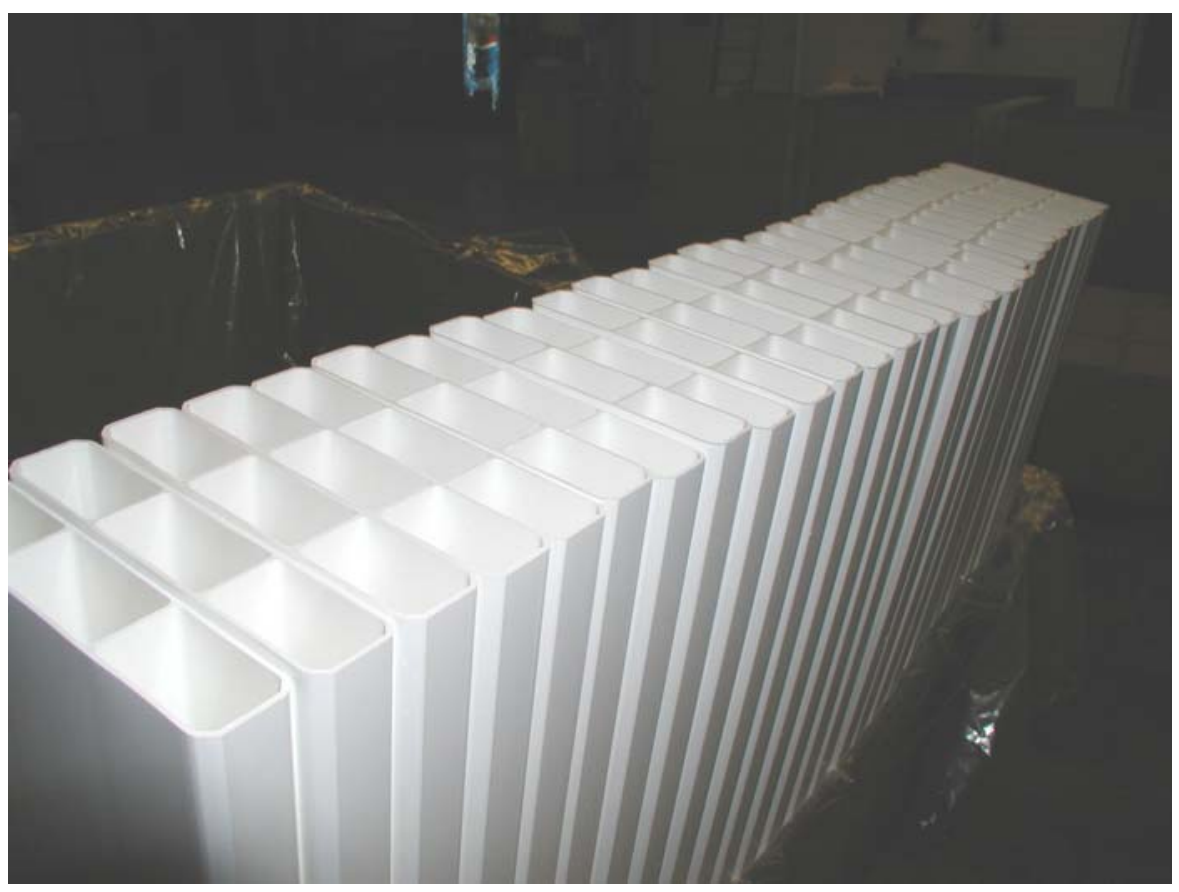

Figure 6. PVC extrusions produced for the NOvA detector by an outside vendor. The cells are 4 $\mathrm{cm}$ wide by $2.5 \mathrm{~cm}$ thick, with $1.5-\mathrm{mm}$ thick external walls and $1.0-\mathrm{mm}$ thick internal walls. The inside corners of the cells are chamfered to improve light collection by the wavelength-shifting fiber readout.

Results of these studies will be used to optimize the parameters of a new baseline design for a totally active detector. This design will be the basis for a revised proposal that the NOvA Collaboration plans to submit to Fermilab in the spring of 2005.

(D.S. Ayres)

\section{b) A New Reactor Neutrino Experiment}

In the presently accepted paradigm to describe the neutrino sector, there are three mixing angles $\left(\theta_{12}, \theta_{23}, \theta_{13}\right)$ that quantify the mixing of the neutrino mass and flavor states. $\theta_{12}$ has been measured by solar neutrino experiments and the KamLAND reactor experiment. $\theta_{23}$ has been measured by atmospheric neutrino experiments and the $\mathrm{K} 2 \mathrm{~K}$ long-baseline experiment, and will be precisely measured by the MINOS experiment. The third angle $\theta_{13}$ has not yet been measured but has been constrained to be smaller than the other two. The best current limit on $\theta_{13}$ comes from the $\mathrm{CHOOZ}$ experiment. It is a function of $\Delta \mathrm{m}^{2}$ and has been measured to be $\sin ^{2} 2 \theta_{13}<0.19$. In order to improve on the $\mathrm{CHOOZ}$ experiment, a new reactor experiment needs more statistics and better control of systematic errors. Increased statistics can be achieved by running longer, using a larger detector, and judicious choice of a nuclear reactor. The dominant systematic errors in an absolute measurement of the reactor neutrino flux, such as cross sections, flux 
uncertainties, and the absolute target volume, will be largely eliminated in a relative measurement with two or more detectors. Good understanding of the relative detector response and the backgrounds is required for a precise relative measurement of the reactor neutrino flux and spectrum. Experiments are being considered which increase the luminosity from the $\mathrm{CHOOZ}$ value of $12 \mathrm{GW}$-ton-year to $400 \mathrm{GW}$-ton-year or more. This will allow a mixing angle sensitivity increase by a factor of 10 . The ability to phase upgrades to achieve a luminosity of $8000 \mathrm{GW}$-ton-year is also being considered. For example, Double CHOOZ has sensitivity to $\sin ^{2} 2 \theta_{13}>0.03$; ANGRA or Braidwood could reach $\sin ^{2} 2 \theta_{13}<0.01$, and experiments with larger detectors could improve another factor of 2 or 3 .

Argonne has been instrumental in the establishment of an International Working Group of physicists who believe that a timely new experiment at nuclear reactors sensitive to $\theta_{13}$ has a great opportunity for discovery. Physicists from Russia, Europe and Japan agreed to work together on a document to present to the physics community. That group produced a white paper which was edited at Argonne, "A New Nuclear Reactor $v$ Experiment to Measure $\theta_{13}$ ", which was published in January 2004.

In October of 2004, ANL led the development of a proposal to the Department of Energy from 7 institutions, "Proposal for U.S. participation in Double Chooz: A new $\theta_{13}$ experiment at the Chooz Reactor." We felt that participation in this experiment was both the best chance to discover a non-zero value of $\theta_{13}$ in a timely way, but also a way to most realistically determine the feasibility of a more sensitive reactor experiment sensitive to $\sin ^{2} 2 \theta_{13}>0.01$. The Double Chooz experiment will run two identical detectors of medium size, containing 12.7 cubic meters of liquid scintillator target doped with $0.1 \%$ Gadolinium. The neutrino laboratory of the CHOOZ experiment, located 1.0 and $1.1 \mathrm{~km}$ respectively form the two cores of the Chooz nuclear power plant will be used again.

The Chooz far site is shielded by about $300 \mathrm{~m}$.w.e. of $2.8 \mathrm{~g} / \mathrm{cm}^{3}$ rocks. An artificial overburden of a few tens of meters height has to be built for the Double Chooz near detector. The required overburden ranges from 53 to $80 \mathrm{~m}$.w.e., depending on the near detector location, between 100 and 200 meters away from the cores. The near and far detectors will be identical inside the PMTs supporting structure. This will allow a relative normalization systematic error of $\sim 0.6 \%$. However, due to the different overburdens (60-80 to 300 m.w.e.), a more sophisticated shielding and veto system will be built for the near detector.

Starting from the center of the target the detector elements are as follows (also see Figure Ic2-1):

$$
\text { - } \quad v \text { target, } 2.7 \mathrm{~m}^{3}
$$




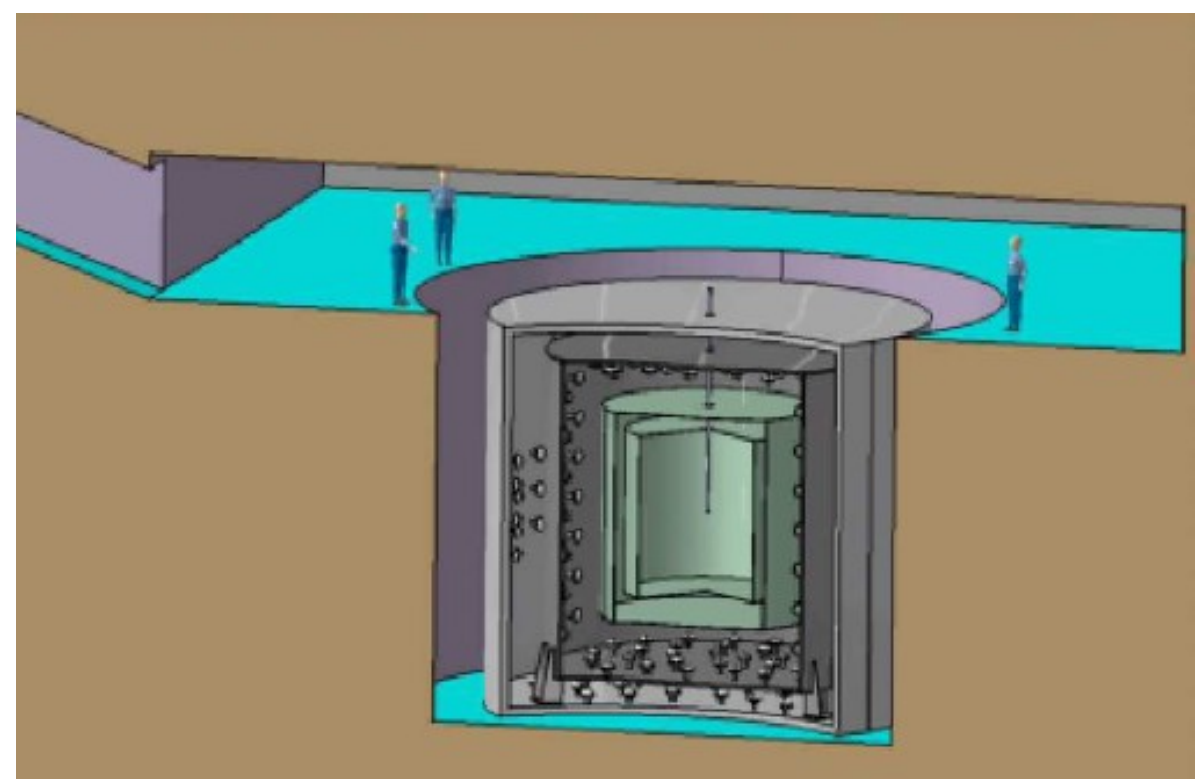

Figure Ic2-1: The Double Chooz near detector at the new underground site. Three regions of the detector can be seen by the inner phototubes. There is also an inner veto and an outer veto (not shown.)

A $120 \mathrm{~cm}$ radius, $280 \mathrm{~cm}$ height, $6-10 \sim \mathrm{mm}$ width acrylic cylinder, filled with $0.1 \% \mathrm{Gd}$ loaded liquid scintillator target

- $\quad \gamma$-catcher, $28.1 \mathrm{~m}^{3}$

A $60 \mathrm{~cm}$ buffer of non-loaded liquid scintillator with the same optical properties as the $v$ target (light yield, attenuation length). This scintillating buffer around the target is necessary to measure the gammas from the neutron capture on $\mathrm{Gd}$, to measure the positron annihilation, and to reject the background from fast neutrons.

- $\quad$ Non Scintillating Buffer, $100 \mathrm{~m}^{3}$

A $95 \mathrm{~cm}$ thick cylindrical buffer of non scintillating liquid, to decrease the level of accidental background (mainly the contribution from photomultiplier tubes radioactivity).

- $\quad$ PMT supporting structure

- Inner Veto system $100 \mathrm{~m}^{3}$

A $60 \mathrm{~cm}$ thick cylindrical veto region filled with liquid scintillator for the far detector, and a slightly larger one (about $100 \sim \mathrm{cm}$ ) for the near detector.

- $\quad$ Outer Veto system (5000 aluminum tubes) 
A four-layer proportional tube system will identify and locate throughgoing muons with $98 \%$ efficiency.

An overview of various predictions for $\theta_{13}$ is given in the White Paper. The conclusion from all these considerations about neutrino mass models is that a value of $\theta_{13}$ close to the CHOOZ bound would be quite natural, while smaller values become hard to understand. Those predictions are depicted graphically in Figure Ic2-2. Double Chooz will cover roughly $85 \%$ of the available parameter space in that graph and cover a similar percentage of those new predictions. We emphasize that a non-zero value for $\theta_{13}$ is expected, unlike the situation in most searches for new physics.

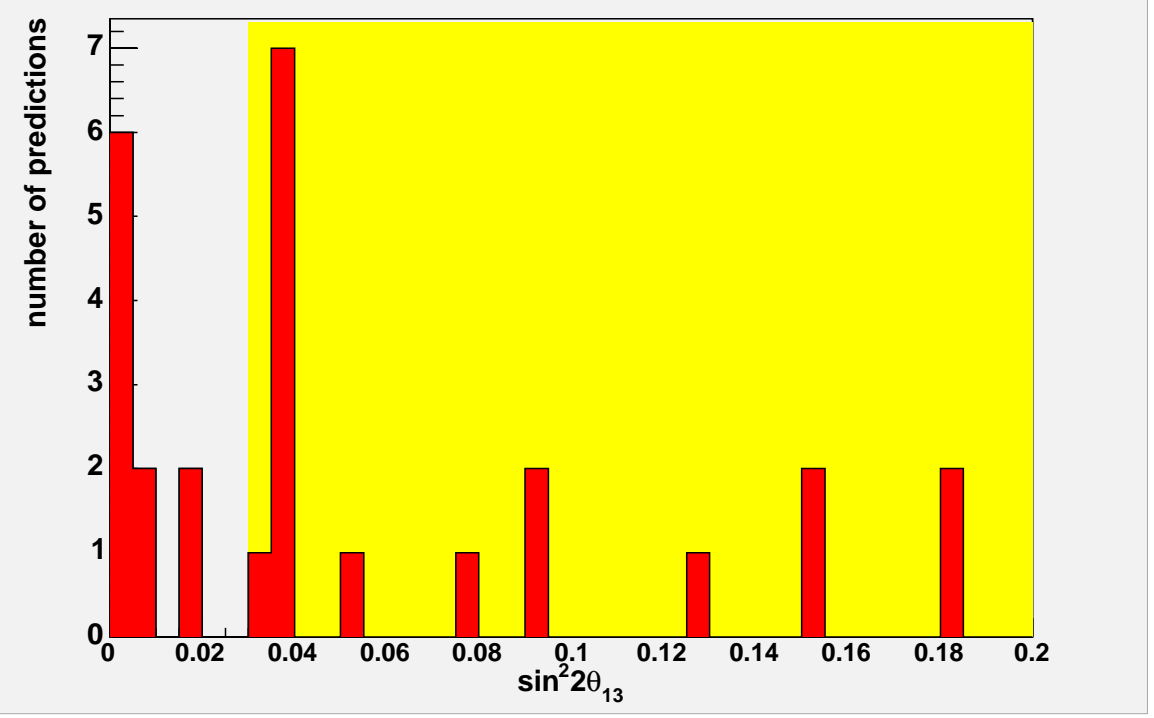

Figure Ic2-2. A graph of a variety of $\theta_{13}$ predictions as summarized in the reactor neutrino White Paper. The region in yellow is accessible to Double Chooz.

\section{Proposed U.S. Systems}

U.S. groups are proposing to contribute three main systems for the Double Chooz experiment: photomultiplier tubes, the outer veto system, and the calibration deployment systems. The PMT system consists of 512 tubes per detector, base electronics, potting and cabling, high voltage, PMT testing, PMT mounting, cleaning and assembly, and finally shipping and installation. The majority of these tasks would be performed by collaborators at Drexel, Louisiana State and Tennessee.

ANL has primary responsibility for the design and construction of an outer veto system. The system will be most extensive for the near detector. A prototype veto tube system has been designed at ANL. The design is loosely based on the structures used in 
the Atlas muon chambers. It will be composed of a collection of 2 inch diameter aluminum extruded pipes with O-ring sealed end-plugs. A resistive wire will be strung down the middle, as shown in Figure Ic2-3. The barrel region will be populated with vertical chambers in which a basic module will consist of 84 chambers (shown in Figure Ic2-4.)

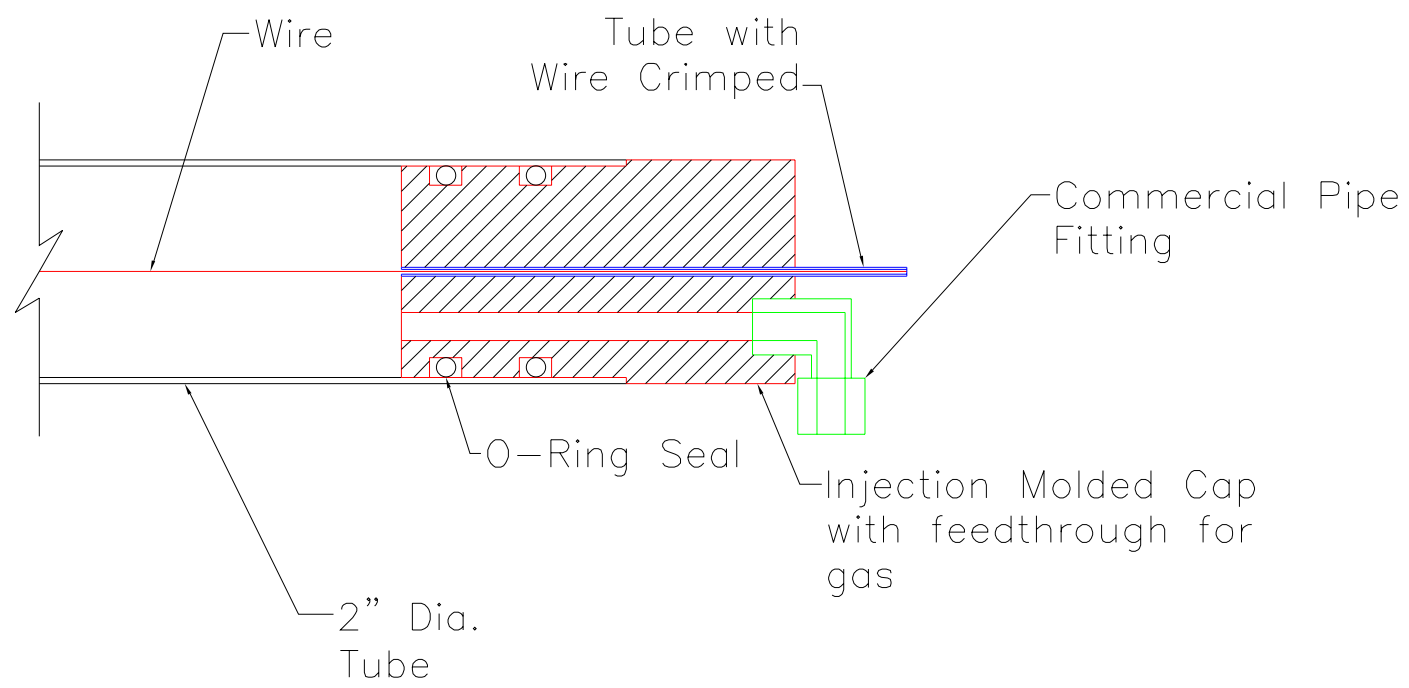

Figure Ic2-3: Schematic design of the veto chamber end cap. The end-gap will be constructed of injection molded plastic with penetratrions for the gas and wire feed-throughs. The end-cap will be double O-ring sealed against gas leakage and the resistive wire will be crimped in place under tension.

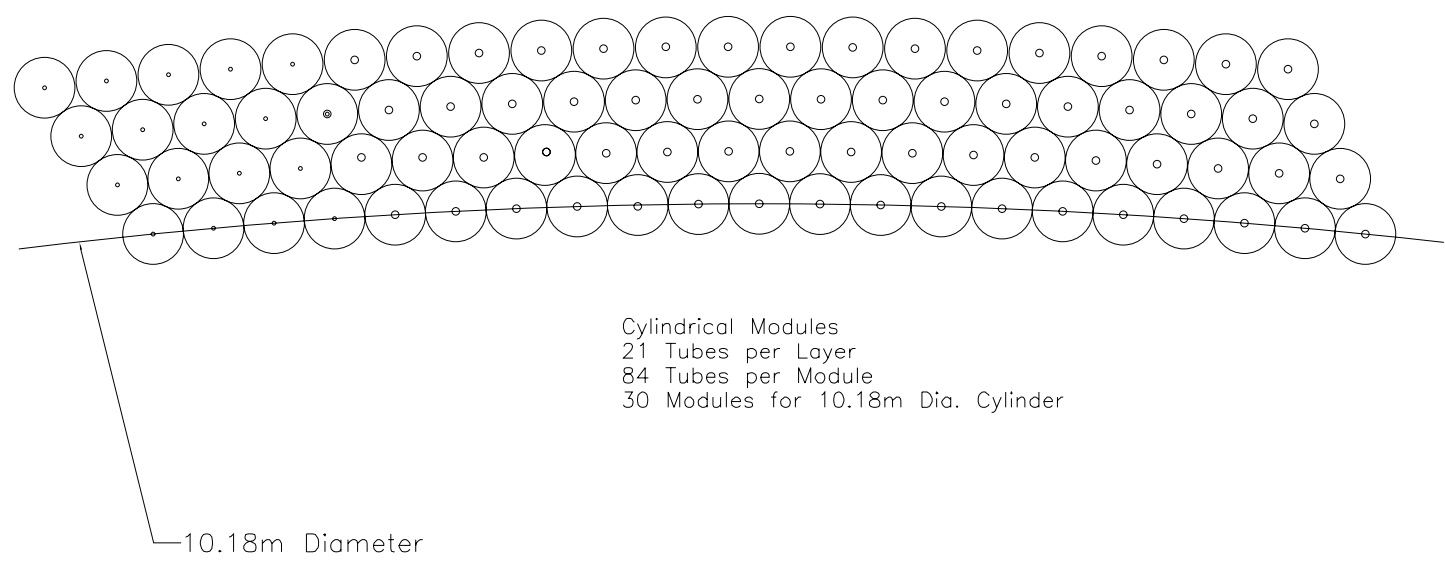

Figure Ic2-4: Schematic of a single barrel region module for the muon veto system. The 84 individual chambers will be epoxied together in the structure shown to exactly encircle the inner detector. The upper and lower planes will be similarly constructed out of 80 chambers and will be flat.

ANL is also involved in the engineering for a calibration deployment system. A variety of beta, gamma, positron and neutron sources are planned. Two designs for the calibration deployment system are being considered: a cable-and-pulley system and a reticulated arm. A winch system will be used to deploy sources along the symmetry axis of the target. 
During Fiscal Year 2006, the fate of the Double Chooz US proposal is expected to be decided by a Neutrino Scientific Advisory Group to HEPAP and NSAC.

(M.C. Goodman)

\section{I.C.5. Cosmic Ray Experiments}

The Very Energetic Radiation Imaging Telescope Array System (VERITAS) is a high energy gamma-ray telescope array with sensitivity in the range $50 \mathrm{GeV}-50 \mathrm{TeV}$ to study extreme astrophysical processes in the universe. The array VERITAS is being built at Kitt Peak in Arizona and is based on the ground-based detection techniques of its predecessor, the Whipple-10 meter optical gamma ray telescope, to explore the universe at the highest photon energies known to be emitted from cosmic sources. The emission of high-energy gamma-rays from cosmic objects always implies the presence of exotic and extreme physical conditions and the detection of this radiation offers a direct probe into these exciting phenomena.

Five Argonne physicists are now contributing to this new astro-particle initiative; Karen Byrum, Steve Magill, Larry Nodulman, Richard Talaga and Bob Wagner as well as one electrical engineer, Gary Drake and one post-doctoral physicist, Elizabeth Hays. We received a joint University of Chicago-Argonne National Laboratory R\&D grant to build a Track Imaging Cherenkov Experiment (TrICE) telescope to attempt to detect primary Cherenkov radiation of charged particles, which can be used to distinguish the composition of cosmic hadrons. But the primary purpose of TrICE is to study the feasibility of using multi-channel photodetectors, to develop front end electronics to be used in conjunction with highly-pixellated future telescopes (VERITAS7) in order to improve night sky background discrimination and to obtain angular resolution.

\section{a) VERITAS:}

Telescope 1

Late summer and fall of 2004 have been very productive for the VERITAS collaboration. The prototype telescope, which is currently located at the base of Mount Hopkins will eventually be moved to Kitt Peak to become part of the 4 telescope array. During summer and fall 2004, the prototype was instrumented with final production version mirrors, camera and electronics. Figure 1 shows the completed Telescope 1. Site development at the future Kitt Peak site also began during this time. Argonne physicists contributed to both the building of the prototype as well as to taking shifts at the 10 meter Whipple telescope and to taking shifts at the 12 meter prototype telescope. We attended 
collaboration meetings, science meetings and have joined multiple physics groups. In September 2004, Liz Hays was hired as a joint University of Chicago / Argonne post doctoral physicist. She immediately became involved with the VERITAS DAQ at the prototype telescope and in a short time has become one of the few DAQ experts.

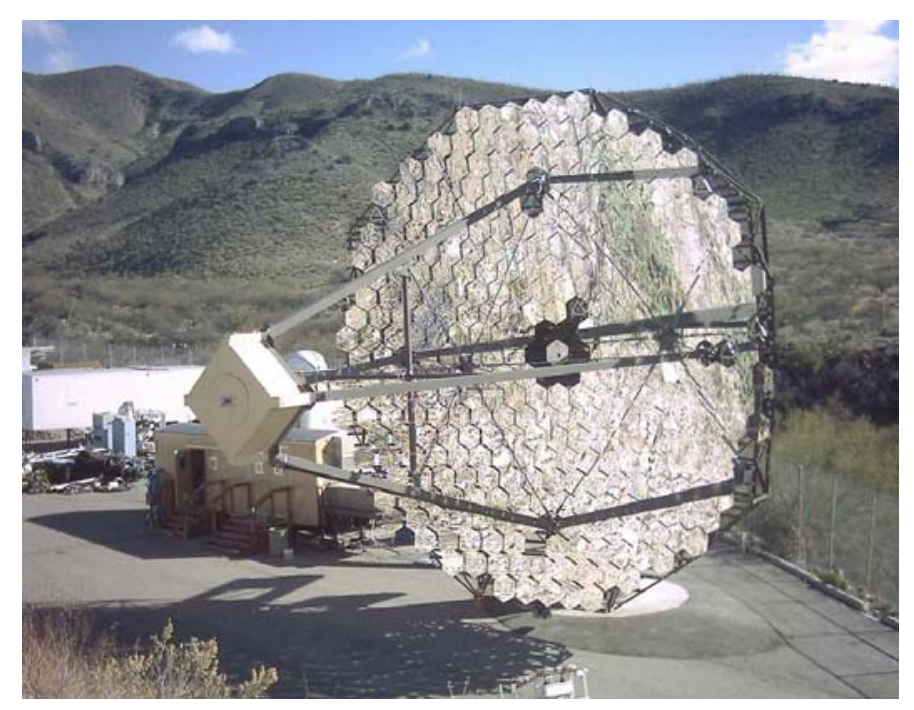

Figure 1: 1a shows Telescope 1 at the base camp on Mount Hopkins instrumented with production mirrors, mounts and camera.

\section{b) TrICE}

\section{$\underline{\text { PMT Studies }}$}

One of the technological challenges of this project is to determine if multi-anode photomultiplier tubes (MAPMTs) can be used in a telescope environment. The key question for this determination are whether MAPMTs have the sensitivity to measure signals of 3100 photo-electrons in a shower in the presence of the high rates of photons from the night sky background.

To study this, Bob Wagner and Karen Byrum have made measurements of the single photo-electron signal on several MAPMTs. We have studied the Hamamatsu R5900-00M64 and the Hamamatsu H8500. We have experience with M64 which is also used by the MINOS experiment, but the pixel spacing of the H8500 is more suited for our angular requirement. Our plan is to understand the performance of the configuration of the voltage divider in the base of the H8500 and attempt to find an optimal configuration for this application. Our measurement setup consists of a dark box containing two PMTs, a blue LED pulsed from an external circuit, a graded neutral density filter wheel driver by a 
stepper motor, a Wratten ND1.0 gelatin neutral density filter and clear fiber to transport LED light pulses to a R580 and the MAPMT. RABBIT electronics are used to measure the charge. Figure 1 shows the spectrums for the M64 tube and for the H8500 tube.
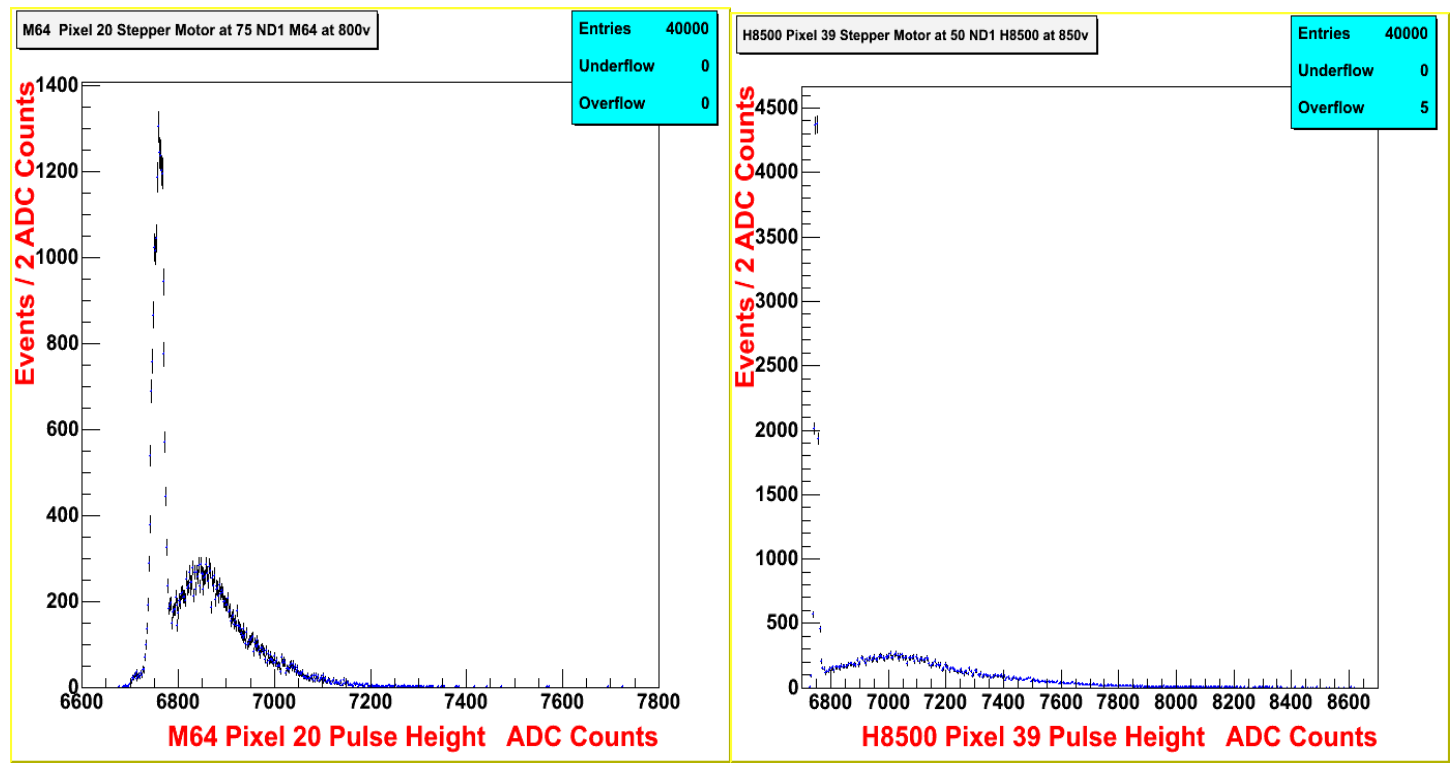

Figure 1. Pulse height spectrum with filter wheel set to allow less than 1 pe. The left figure is the spectrum for the M64 PMT and the right figure is for the H8500 PMT.

We also studied crosstalk on the M64 and found this to be less than 3\% which is consistent with previous measurements from the MINOS experiment. Initial measurements of the $\mathrm{H} 8500$ seem of similar scale.

We have also made preliminary linearity measurements with and without a simulated effective night sky background. To simulate the night sky background, we used an incandescent bulb inside the black box setup. The linearity measurement records the measured ADC counts at each filter wheel setting for the case of the bulb off (no NSB) and compares this to a measurement with the bulb on. The inferred ADC counts for bulb on (non-zero NSB) are normalized to the value of the R580 which is shielded from the simulated NSB. Figure 2 plots the curve of relative response vs number of photoelectrons for each DC current where we have assumed the conversion of $1 \mathrm{nA}=113.6 \mathrm{k} \mathrm{p.e./s}$ to give the equivalent p.e./s that our bulb illumination gives. Initial results show that an incandescent bulb shining on our photocathode has relatively small effect on the gain, even at high illumination. We plan to obtain a better calibration of the effect of a simulated NSB by using the current observed by tube in the presence of the actual NSB. 


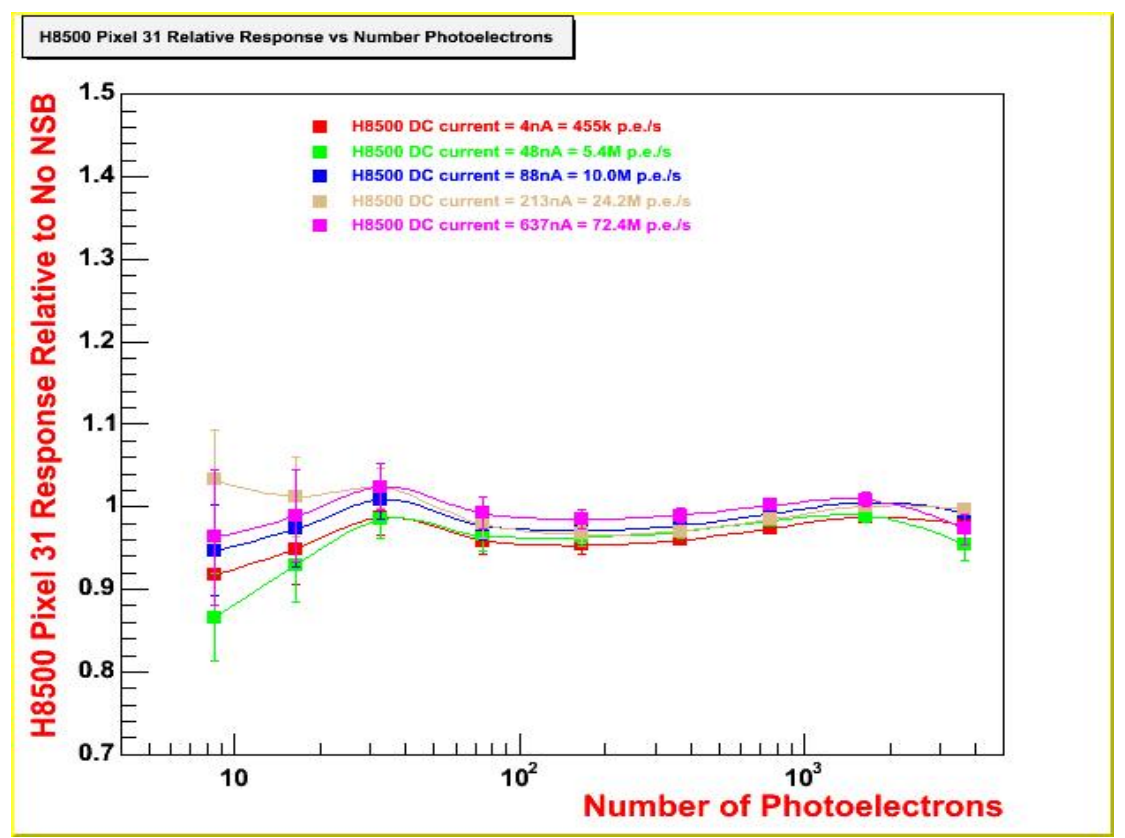

Figure 2. The response of one pixel on the H8500 PMT as a function of the number of photoelectrons pulsed through the filter wheel at different settings of simulated NSB.

\section{TRICE DAQ}

Liz Hays has taken over the development of data acquisition software for the TRICE project. The DAQ uses MINOS pre-production electronics boards readout by a VMIC 7766 processor. This processor is also used by the VERITAS experiment. This design was chosen to closely overlap the TrICE DAQ software development with the VERITAS experiment. The VMIC processor uses real time Linux as its operating system. During Jul - Dec 04, software was developed to calibrate the electronics. Figure 3 shows the raw DAC-ADC data points used to compute lookup tables constants (LUTs)

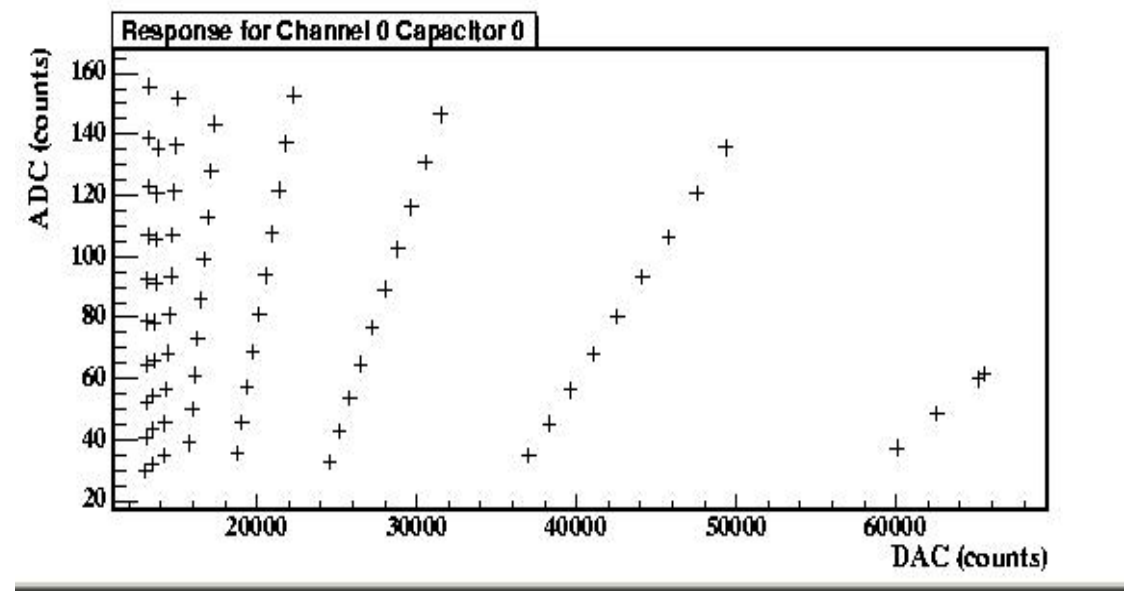

Figure 3. The raw DAC-ADC data points used for calculating LUT constants. 
which are used for linearizing the data for all 8 ranges. Data is only shown for one of the 4 capacitors. Figure 4 shows the linearized ADC values resulting from reading DAC data through the LUTs. This is an average for reading through all 4 capicitors multiple times for each DAC setting.

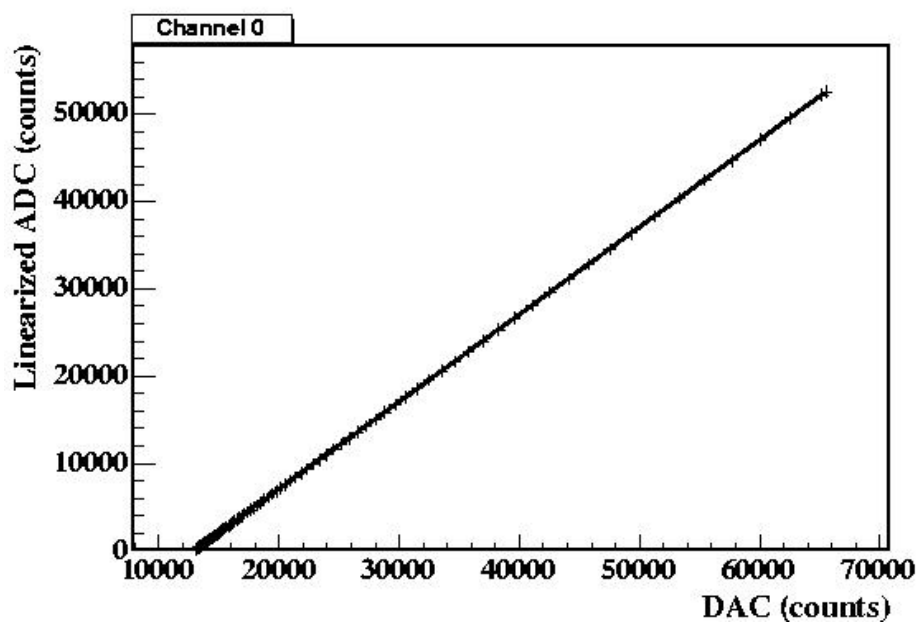

Figure 4. The linearized ADC values resulting from reading DAC data through the LUTs.

\section{$\underline{\text { MC Studies }}$}

Steve Magill and Liz Hays worked on developing a TrICE Monte Carlo using the CORSIKA generator. The CORSIKA Air Shower Monte Carlo generator was obtained from its author and set up on the ANL server. Input options for Cerenkov photon generation and storage were included. The program can be run with a separate Cerenkov photon output file with the option of making an ntuple with the Cerenkov photons and the CORSIKA output is transformed into a format which can be read by a detector simulation program written by Scott Wakely and Simon Swordy of UofC.

Figure 5 is a plot of simulated photons from a $100 \mathrm{TeV}$ iron induced air shower in the TRICE 1600 pixel camera. The first interaction occurs at a height of $30 \mathrm{~km}$ - the detector sits at $200 \mathrm{~m}$ above sea level. The photons are integrated over the duration of the shower $\sim 30 \mathrm{~ns}$. The majority of the photons arrive in the first $20 \mathrm{~ns}$. The binning is very close to the actual pixel size of the H8500. The top plot shows the photons from the longer mirror path. The bottom plot shows the "trigger" signal which arrives through the 
Fresnel lens $\sim 30 \mathrm{~ns}$ ahead of the mirror signal. The detector location has been offset from the shower core by $80 \mathrm{~m}$, where the DC signal is maximized, causing the image to look extended along the $\mathrm{X}$ axis. Quantum efficiency has not been included in these plots so the actual detected photon numbers will get decreased by a factor of 3 or 4 . This figure includes no background noise or time jitter from the electronics.
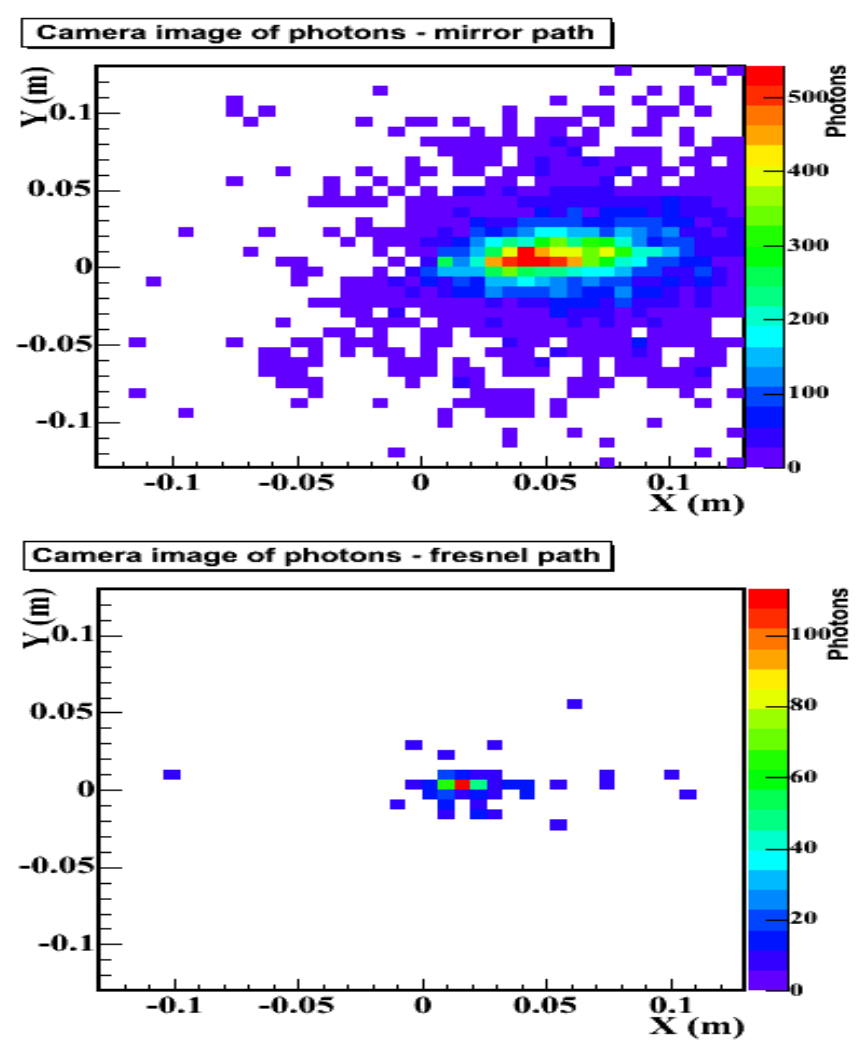

Figure 5.

Figure 6 is an idealized extraction of the DC signal. The plot is a projection of the camera image along the y axis. The black line indicates all the photons that hit the camera. The red line indicates the DC signal we are looking for. The blue and green dashed lines show the collected signal with two cuts applied. The first excludes pixels not lying along the shower axis, and the second is a time cut removing photons that arrive before the $8 \mathrm{~ns}$ delay in arrival for the DC light due to longer time of travel in the atmosphere. 


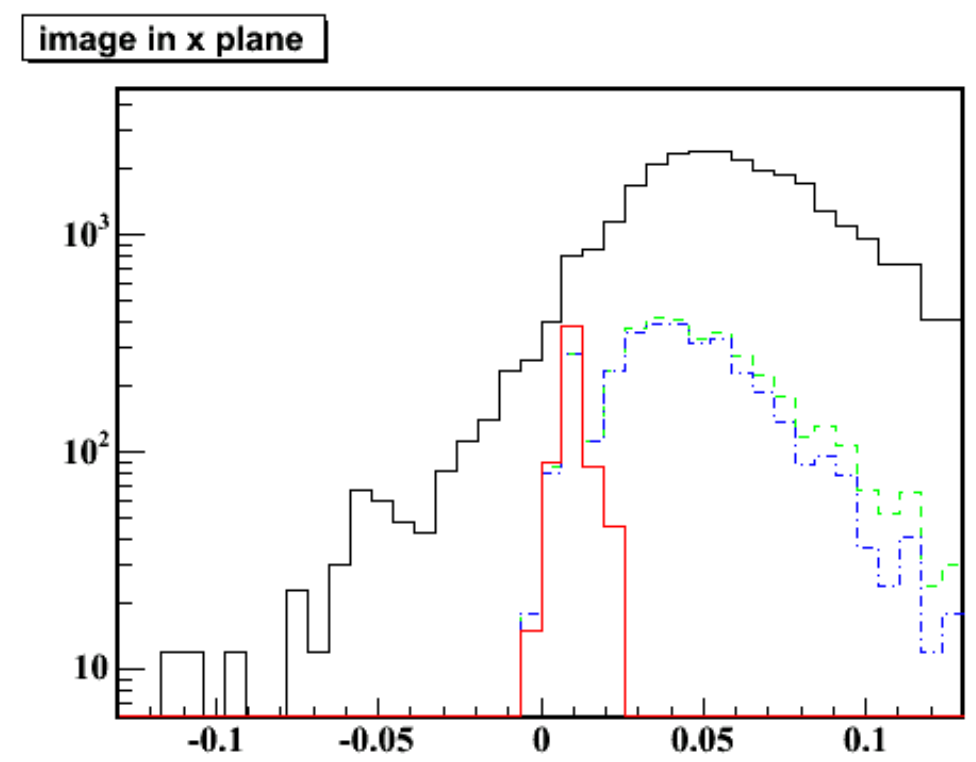

Figure 6.

\section{$\underline{\text { Site Development }}$}

We performed preliminary night sky background measurements at two possible sites and selected the site with the darkest night sky background. We measured the current on the R580 anode and on the H8500 "or" of the 64 pixels as a function of HV. The PMTs were shielded with a large baffle. We expect this current to be an upper limit on the night sky background our camera will view. Figure 7 shows typical H8500 signals with the voltage set to $540 \mathrm{~V}$. With this voltage, the "or" of the 64 pixels recorded a current of 65 microAmps.

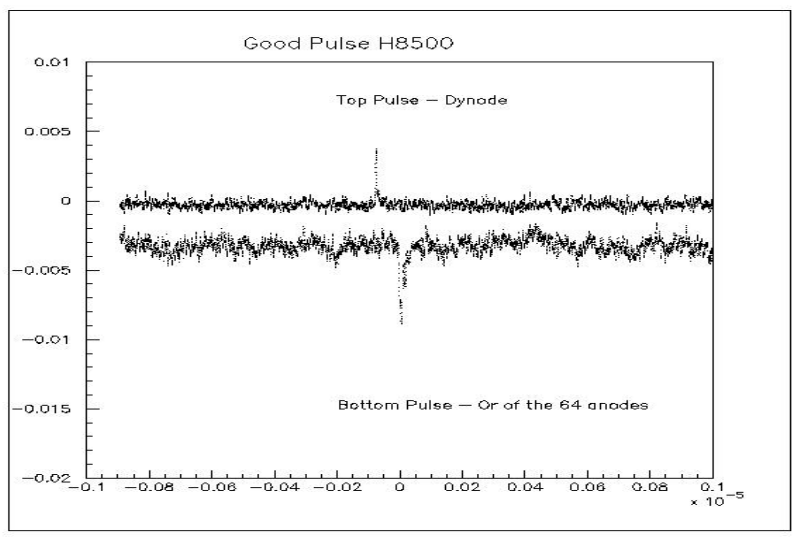

Figure 7. Scope trace of a typical H8500 signal taken outside on a dark night. The high voltage was set to $540 \mathrm{~V}$ and the current from the "or" of all 64 anodes measured 64uAmps. 
In the second half of 2004, we started site development. After finalizing our site selection in early summer, we received NEPA approval and brought power to the site in late august. We completed the design of the mirror and mount of the telescope and ordered a trailer for housing computers and electronics and an enclosable structure for housing the telescope. Figure 8 shows a schematic of the telescope mount.

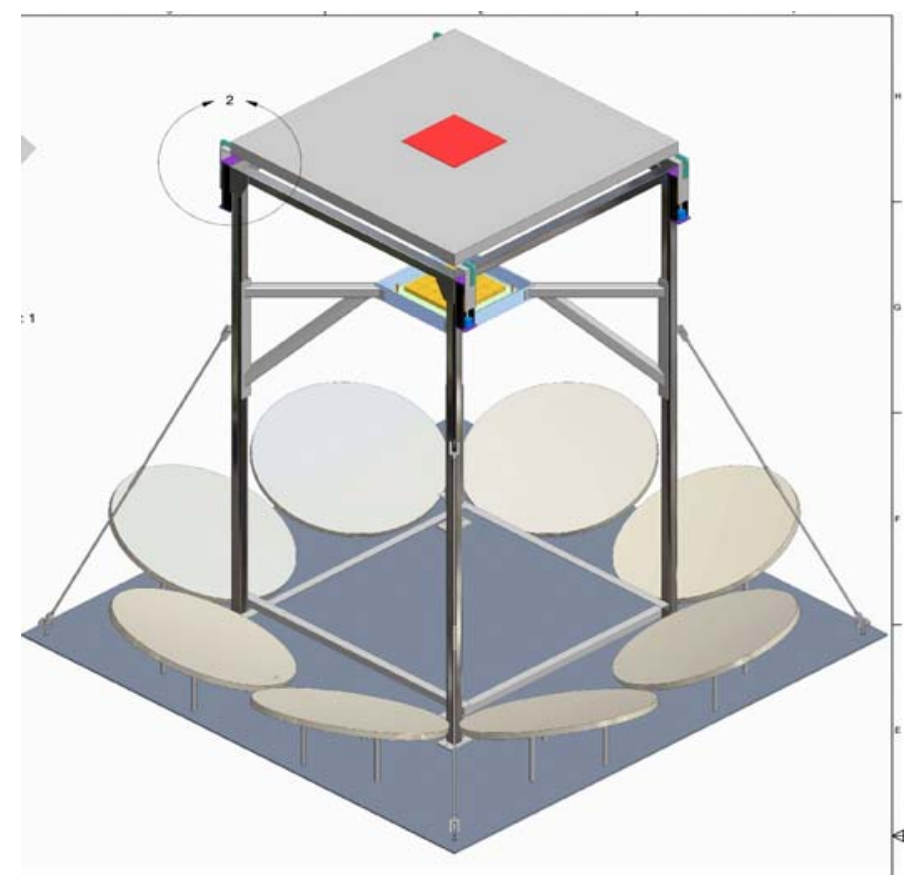

Figure 8. Schematic drawing of TrICE telescope.

(K. Byrum, R. Talaga, S. Magill, R. Wagner)

\section{c) The Auger Experiment}

The goal of the Pierre Auger Observatory project is to study the highest energy cosmic rays. At energies near $10^{20} \mathrm{eV}$, the deflection of protons by magnetic fields between and within galaxies is expected to be small, so that showers from such particles should point back to their source. Furthermore, cosmic ray interactions on cosmic microwave background $\left(3^{\circ} \mathrm{K}\right)$ photons should cause significant energy loss (GZK cutoff) for energies above about $5 \times 10^{19} \mathrm{eV}$ and distances greater than $\sim 150 \mathrm{M}$ light years. Yet there are few known sources that could produce such high energy particles within this distance, and there is evidence from several experiments that such ultra high energy cosmic ray showers have been detected. These showers do not point to one of the known sources. Thus, the observations may suggest new particle physics, new astrophysics, or both. 
The initial planning for the Pierre Auger Observatory assumed detection of cosmic ray showers with angles up to $60^{\circ}$ from the zenith. More recently, it has been realized that nearly horizontal showers will also be recorded. Most of these are dominated by muons, since the electromagnetic component is absorbed before reaching the detectors. However, neutrino interactions could produce showers with a significant number of electrons and positrons, even though they appear to have gone through the equivalent of several atmospheres of air or the nearby Andes mountain range. Thus, the Auger experiment is also sensitive to the highest energy cosmic ray neutrinos.

Cosmic ray showers are observed in the Auger experiment with two independent detector systems: a surface array composed of water Cerenkov tanks (live time $\sim 100 \%$ ) and a telescope array of fluorescence detectors (live time $\sim 10 \%$ ), similar to those in the Fly's Eye experiment. Hybrid events detected with both systems allow, for the first time, cross checks of energy calibrations and tests for systematic effects resulting in a significant improvement of cosmic ray energy determination. For example, there is evidence for differences in energy calibrations in previous experiments that used different types of detectors. The southern Auger observatory is under construction near Malargue, Argentina, with completion expected in 2006. A northern Auger site in the western US will be proposed in the near future in order to attain full sky coverage; this would provide important information about the origins of these cosmic rays.

Argonne physicists hope to join the Pierre Auger collaboration and to be involved in one or more major aspects of the northern site construction. One of us has worked for some time on the water Cerenkov tanks with physicists at Fermilab. At this time our group is preparing to make measurements with ANL electron accelerators on the fluorescence yields to calibrate cosmic ray shower energies. We are also performing some analysis of pulse shapes from the surface array in Malargue.

The fluorescence detectors record shower development in the atmosphere. The total light yield is obtained after correction for atmospheric conditions and geometric effects. The relationship between total light yield and the shower energy relies on measurements with a few electron energies from many years ago and has an estimated uncertainty of $\pm 15 \%$. A new set of data for electron energies above $50 \mathrm{MeV}$ was recently obtained from an experiment at Frascati. The goal is to significantly improve the cosmic ray energy uncertainty associated with the fluorescence detectors in Auger and elsewhere. However, measurements at lower energies are required to reduce the overall cosmic ray energy uncertainty to $\pm 5 \%$, because it is expected that approximately half the light yield results from energies below $10 \mathrm{MeV}$.

Tests were made with the Argonne Wakefield Accelerator (AWA) to determine backgrounds for measurements of fluorescence light yields in air for electron energies of $\sim 4-13 \mathrm{MeV}$. The experiment would use the same apparatus constructed for a similar 
experiment at Frascati (AIRFLY) at higher energies. Two test runs occurred, and it was concluded that the background rates could be made acceptable. It was found that better focusing of the beam near the apparatus would be required, and work is in progress to accomplish this by adding a quadrupole triplet and a pair of steering magnets to the AWA beam line. This effort is in collaboration with University of Chicago physicists.

(H. Spinka) 


\section{THEORETICAL PHYSICS PROGRAM}

\section{II.A. THEORY}

\section{II.A.1. Transverse Momentum Distribution of Upsilon Production}

Ed Berger continued his work with Jianwei Qiu and Yi-li Wang of Iowa State University on the production dynamics of $\Upsilon$ states in hadronic reactions, applicable over the full range of values of transverse momentum. Their results, and a recent comparison with FNAL Tevatron Run-II data, are shown in Fig. 1 and confirm that the all orders resummable part of initial-state gluon showering provides the correct shape of the transverse momentum distribution in the region of small transverse momentum. Their research is summarized in Physical Review D71, 034007 (2005) and in hep-ph/0411026, a contribution to DPF 2004: the Annual Meeting of the Division of Particles and Fields (DPF) of the American Physical Society (APS), Riverside, California, 26-31 Aug 2004. Berger et al's work was featured as the "Fermilab Result of the Week" in the Thursday, January 13, 2005, issue of Fermilab Today.

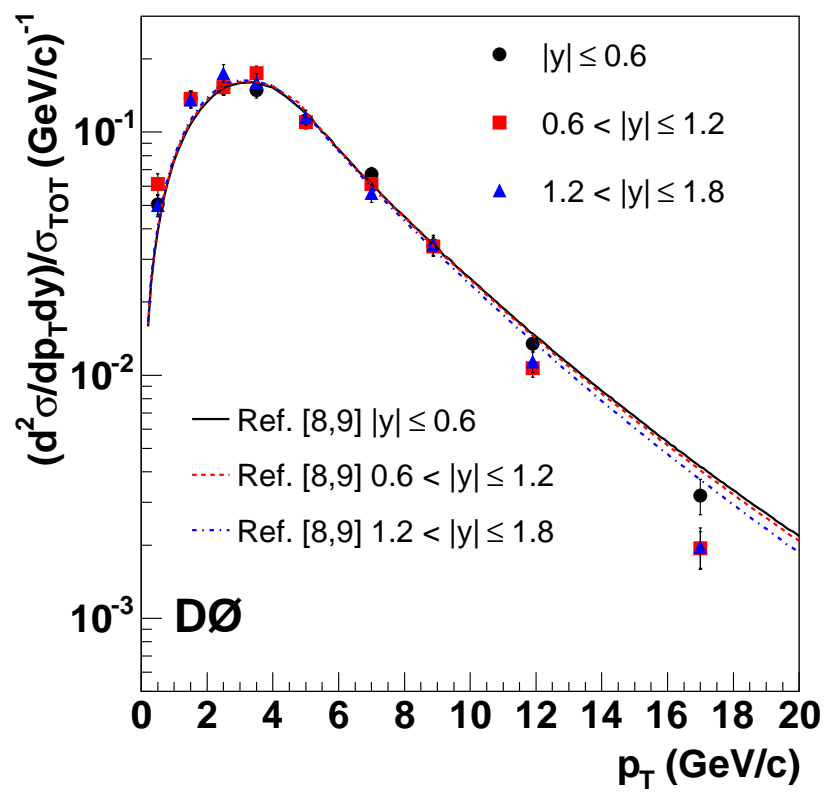

Figure 1. The predictions of Berger, Qiu, and Wang for the transverse momentum $p_{T}$ distribution of $\Upsilon$ production are shown (denoted Refs. 8 and 9) along with FNAL Run-II data from the D0 collaboration, from hep-ex/0502030.

(E. L. Berger) 


\section{II.A.2. Determination of the HWW and HZZ Couplings at the LHC and the ILC}

Continuing their investigation of Higgs boson production, Ed Berger and John Campbell focused on the accuracy with which the Higgs boson coupling to weak bosons may be determined from data at the CERN Large Hadron Collider (LHC) and the International Linear Collider (ILC). They estimated that an accuracy of approximately $10 \%$ may be achievable after 200 inverse femtobarns of integrated luminosity are accumulated at the LHC. For comparison, at a $500 \mathrm{GeV}$ electron-positron linear collider, the expected accuracies are 3\% in the HZZ coupling and 3\% to 7\% in the HWW coupling after 500 inverse femtobarns of integrated luminosity are accumulated. Reports of this research appear in Physical Review D70, 073011 (2004) and in hep-ph/0408259, to appear in the Proceedings of 12th International Conference on Supersymmetry and Unification of Fundamental Interactions (SUSY 04), Tsukuba, Japan, 17-23 June 2004.

(E. L. Berger)

\section{II.A.3. Bounds on the Gluino Mass from a Global Parton Density Analysis}

In hep-ph/0408217, ANL-HEP-CP-04-75, a paper to be published in the Proceedings of 12th International Conference on Supersymmetry and Unification of Fundamental Interactions (SUSY 04), Tsukuba, Japan, 17-23 June 2004, Ed Berger summarized research he did with Pavel Nadolsky, Fred Olness of Southern Methodist University, and Jon Pumplin of Michigan State University [Physical Review D71, 014007 (2005)]. In their work, they obtain model-independent bounds on the existence and mass of color-octet fermions (gluinos) based on a fit to the complete CTEQ6 set of inclusive hadron scattering data (1811 points). The data are characterized by high precision and cover a broad range in $x$ and four-momentum transfer $Q$. Berger et al include SUSY contributions to the Tevatron jet production cross sections and use the full CTEQ error analysis to obtain their bounds. They determine that the gluino mass must be greater than $12 \mathrm{GeV}$ if the strong coupling strength, evaluated at the mass of the $\mathrm{Z}$ boson, is 0.118 , with smaller values of mass allowed for larger values of $\alpha_{s}\left(M_{Z}\right)$. The PDF analysis of current hadron scattering data is not sensitive to gluinos with mass above the weak-scale, but inclusion of a gluino with mass of $100 \mathrm{GeV}$, or so, slightly improves the description of jet data at high transverse energy $E_{T}$. The study of Berger et al is complementary to those in which bounds on the gluino mass are derived from LEP data, and it demonstrates the potential for PDF analysis to independently constrain new physics, in the next few years, once high precision data extend to larger values of $Q$.

(E. L. Berger) 


\section{II.A.4. Physics Interplay of the LHC and the ILC}

As members of the LHC/ILC study group, Ed Berger, Carlos Wagner, and Tim Tait contributed to the document "Physics Interplay of the LHC and the ILC", hepph/0410364, ANL-HEP-PR-04-108, a 472 page document to be published in Physics Reports.

This report addresses the possible synergies between the LHC and ILC in testing the Standard Model and in discovering and determining the origin of new physics. Mutual benefits for the physics programs at both machines can occur at the level of a combined interpretation of Hadron Collider and Linear Collider data, and at the level of combined analyses of the data, where results obtained at one machine can directly influence the way analyses are carried out at the other machine. Possibilities exist whereby physics discoveries at the ILC can influence the way experiments and triggers are designed during the latter years of LHC operation. Topics included in the report include the physics of weak and strong electroweak symmetry breaking, supersymmetric models, new gauge theories, models with extra dimensions, and electroweak and QCD precision physics.

(E. L. Berger, C.E.M. Wagner, and T.M.P. Tait)

\section{II.A.5. Lattice Computation of Spin Correlations in NRQCD Color-Octet Matrix Elements}

The motivation for this study and previous work on the subject by G. Bodwin, J. Lee (Korea University), and D. Sinclair are described in the reports for the periods July 1, 2003--December 30, 2003 and January 1, 2004--June 30, 2004.

G. Bodwin, J. Lee, and D. Sinclair have shown, in a perturbative analysis, that non-spin-flip NRQCD color-octet matrix elements contain contributions that scale as $\left[\alpha_{s}(\Lambda)\right]^{2}(\Lambda / m)^{2} \log ^{2}(\Lambda / m)$ and that the spin-flip matrix elements contain contributions that scale as $\left[\alpha_{s}(\Lambda)\right]^{2}(\Lambda / m)^{4}$. Here, $\Lambda$ is the cutoff of the effective field theory NRQCD, and $m$ is the heavy-quark mass. These contributions that scale as powers of $\Lambda$ potentially spoil the velocity-scaling rules of NRQCD if $\Lambda$ is chosen to be significantly larger than $m$. On the other hand, $\Lambda$ scales as $1 / a$, where $a$ is the lattice spacing. Therefore, if $\Lambda$ is taken too small, the lattice spacing is too coarse to reproduce the physics of the quarkonium system accurately. This latter issue is significant for charmonium, but is less important for bottomonium.

Recently, G. Bodwin, J. Lee, and D. Sinclair have addressed the problem of

these conflicting criteria for the choice of the cutoff $\Lambda$ in the case of charmonium by 
modifying the rules for computing the lattice NRQCD heavy-quark Green's functions. The modifications increase the effective value of $\Lambda$ in the operator matrix elements, but not in the overall evolution of the quarkonium state. Hence, they controls the contributions to the matrix elements that scale as powers of $\Lambda$, but do not affect the accuracy with which the quarkonium is described on the lattice. The modified Green's functions reproduce the spectrum results for charmonium that have been published by the NRQCD collaboration and also lead to values for the matrix elements that are consistent with the velocity-scaling rules of NRQCD.

A paper describing these results (ANL-HEP-PR-05-9) is in preparation.

(G. T. Bodwin and D. K. Sinclair)

\section{II.A.6. NRQCD Factorization vs. the Color-Evaporation Model}

The color-evaporation model (CEM) is the only viable competitor to the NRQCD factorization approach for describing quarkonium production. In the CEM, one computes quarkonium production cross sections by calculating the perturbative rate for $c \bar{c}$ production below open charm threshold, summed over spin and color, and multiplying by a phenomenological constant that is different for each quarkonium state.

G. Bodwin, in collaboration with Eric Braaten (Ohio State University) and Jungil Lee (Korea University), has begun an analysis of the relationship of the CEM to the NRQCD factorization approach. One key to this analysis is the realization that one can calculate the rate for $c \bar{c}$ production below open charm threshold, which appears in the CEM cross sections, by using the NRQCD factorization formula for the case of $c \bar{c}$ production. Since the short-distance coefficients that appear in the NRQCD formula for $c \bar{c}$ production are identical to the short-distance coefficients that appear in the NRQCD factorization formula for quarkonium production, one can use the result of this calculation to obtain the constraints on the NRQCD matrix elements that are implied by the model assumptions of the CEM. These constraints manifest themselves as predictions for the relative sizes of the NRQCD matrix elements.

Bodwin, Braaten, and Lee find that the CEM constraints satisfy the NRQCD velocity-scaling rules in the ratio of the most important matrix elements for $J / \psi$ production, but not for the ratio of the most important matrix elements for $\chi_{c}$ production. The CEM constraints also impose incorrect color factors on the matrix elements that are particularly at variance with the correct color factors in the case of $\chi_{c}$ production. The conclusion is that the CEM models the evolution of a heavy quark-antiquark pair into quarkonium in a way that is very different from NRQCD. 
Nevertheless, the CEM and NRQCD factorization both fit the data reasonably well, with comparable values of $\chi_{c}$ per degree of freedom in many cases. However, one major difference is that the CEM requires $k_{T}$ smearing of the momentum of the initial partons in order to fit the data, while NRQCD factorization does not. $k_{T}$ smearing is a simple (perhaps simplistic) model for the effects of multiple soft-gluon emission in which Gaussianly distributed transverse momentum is added to the momentum of the initial partons. Bodwin, Braaten, and Lee are now in the process of calculating the effects of $k_{T}$ smearing on the NRQCD factorization predictions to see if the amount of $k_{T}$ smearing that is required in the CEM (about $1.6 \mathrm{GeV}$ ) is compatible with NRQCD factorization.

(G. T. Bodwin)

\section{II.A.7. CERN Yellow Report on Heavy-Quarkonium Physics}

The Quarkonium Working Group (QWG) is an international organization that consists of more than 150 theorists and experimenters. Its aim is to promote discussions on theory and experiment in heavy-quarkonium production, decay, and spectroscopy and to foster interactions between theorists and experimenters working in these fields.

During the past two years, with the aim of providing a resource to the HEP community, the Quarkonium Working Group has written a detailed review of the status of theory and experiment in quarkonium physics. This massive (521 page) review (hepph/0412158) will be published as a CERN Yellow Report. It covers common theoretical tools, a description of experiments and detectors, quarkonium spectroscopy, quarkonium decays, quarkonium production, precision measurements in quarkonia, quarkonium production in media, and future opportunities in quarkonium physics. G. Bodwin is one of the principal authors of the Production chapter, which runs nearly 70 pages in length, and also contributed to the Outlook chapter and to the general structure and content of the review.

(G. T. Bodwin)

\section{II.A.8. QWG Topical School on Heavy Quarkonia}

The nature and goals of the Quarkonium Working Group (QWG) are described in the preceding article. During October 8-11, 2004, the QWG held the Topical Seminar School on Heavy Quarkonia at Accelerators: New Theoretical and Experimental Techniques, which took place at the Institute of Theoretical Physics (ITP) in Beijing, China. The School was organized with the aim of educating young physicists at the graduate-school level in the latest advances in quarkonium physics, and it attracted about 
80 participants. G. Bodwin delivered a series of lectures at the School on an Introduction to NRQCD and Inclusive Quarkonium Production (theory versus experiment). Details of the program and the lecture transparencies can be found at www.qwg.to.infn.it/TSoct04/index.html.

(G. T. Bodwin)

\section{II.A.9. Lattice QCD}

While lattice QCD simulations to study the properties of hadrons and their interactions, and to study the properties of hadronic matter, nuclear matter and the quarkgluon plasma, continued to dominate our research, we also started to apply these methods to the study of physics beyond the standard model.

We prepared code to perform Monte Carlo simulations of Born-Infeld field theories on a space-time lattice. Born-Infeld field theories (non-linear electrodynamics) have recently gained attention as theories, which appear to have string/brane solutions. Because we work in Euclidean space, the Born-Infeld action is positive, which allows the use of standard Monte Carlo (importance sampling) methods. Introducing the current source needed to study the screening properties of this theory introduces a potential sign problem. However, the theory is such that there are ways of avoiding the exponential time problem associated with this sign problem.

We are continuing our studies of 3-flavour QCD at a finite quark-number chemical potential using only the magnitude of the fermion determinant. In the small chemical potential (small quark-number density) regime, close to the finite temperature transition from hadronic/nuclear matter to a quark-gluon plasma, there is mounting evidence that the position and possibly the nature of this transition are not affected by the omission of the phase of the determinant. We are studying this transition using Binder cumulant methods to determine its nature and look for signs of the expected critical endpoint. Particular attention is being paid to understanding the dependence of these cumulants on the 'time' increment $d t$ used in our simulations.

Our studies of the spin dependent colour octet matrix elements contributing to the decays of charmonium and bottomonium are showing preliminary evidence for the veracity of the velocity scaling rules of NRQCD. More studies are underway to better understand the charmonium case where we cannot lower the ultra-violet cutoff as low as is desirable for NRQCD. These matrix elements we are calculating are related by crossing to those presumed responsible for heavy quarkonium production at high transverse momenta at the Tevatron.

We are continuing to collect statistics in a study of the properties of the finite temperature chiral transition for 2 flavours of massless quarks. These simulations are 
being performed on $12^{3} \times 8,16^{3} \times 8$, and $24^{3} \times 8$ lattices. Here we are using a lattice QCD action, modified to allow simulations at zero quark mass. Our goal is to determine the critical exponents at this finite-temperature chiral phase transition and determine its universality class.

(D. K. Sinclair)

\section{II.A.10. Warped Fermions and Precision Tests}

In "Warped Fermions and Precision Tests", published in Phys. Rev. D71, 015010 (2005), T.M.P. Tait and C.E.M. Wagner, along with M. Carena (FNAL), A. Delgado (Johns Hopkins U and CERN), and E. Ponton (FNAL and Columbia U), have investigated the effects of brane kinetic terms on theories with a warped extra dimension. Such theories have a novel solution to the hierarchy problem, and may be dual to strongly coupled conformal four dimensional theories. Traditionally, such theories have the entire Standard Model confined to a brane, while gravity propagates in the bulk. However, the solution to the hierarchy problem demands only that the Higgs be confined to a brane, and flavor physics and grand unification provide powerful motivation to consider the case in which the Standard Model fields are also in the bulk. While theoretically attractive, this scenario has strong constraints from electroweak precision physics, which typically require that the Kaluza Klein modes have masses greater than $10 \mathrm{TeV}$, beyond the reach of near future colliders.

In this work, we have examined the effect of kinetic terms for the fermions, which are localized on one of the branes. These effects have dramatic effects on the phenomenology, and act to reduce the Kaluza-Klein masses, soften strong coupling to brane fields, and improve the electroweak fit. This allows us to move the top quark further away from the Higgs brane, and improve the electroweak fit for smaller KaluzaKlein mode masses. Ultimately, we find that Kaluza-Klein mode masses as low as a few $\mathrm{TeV}$ are allowed when brane kinetic terms are included, and these masses are expected to be within reach of the $\mathrm{LHC}$.

(T.M.P. Tait and C.E.M. Wagner)

\section{II.A.11. The Supersymmetric Origin of Matter}

The nature of the dark matter and the origin of the baryon asymmetry are two of the most important questions at the interface of particle physics and cosmology. The Standard Model (SM) of particle physics, which does an excellent job explaining the results of collider experiments, is unable to explain either of these phenomena. This situation can be improved by extending the SM to include supersymmetry. In the minimal supersymmetric extension of the Standard Model, the MSSM, the lightest supersymmetric particle (LSP) is stable as a result of R-parity. If this particle is the 
lightest neutralino, a mixture of the superpartners of the neutral gauge and Higgs bosons, its mass and couplings are of the right size to generate the observed dark matter density. The MSSM can also explain the baryon asymmetry by the mechanism of electroweak baryogenesis (EWBG), in which the asymmetry is generated during the electroweak phase transition in the early Universe.

The requirement of electroweak baryogenesis imposes strong constraints on the parameter space of the MSSM. EWBG only works if the electroweak phase transition is strongly first-order. Within the MSSM, this is the case only if the Higgs boson is relatively light, $m_{h} \lesssim 120 \mathrm{GeV}$, and one of the scalar top quarks has mass less than that of the top quark, $m_{\tilde{t}_{1}} \lesssim 175 \mathrm{GeV}$. A new source of CP violation beyond the phase in the CKM matrix is also needed for EWBG to generate the baryon asymmetry. The most efficient source in the MSSM comes from the chargino sector, provided the charginos have mass below about $500 \mathrm{GeV}$ and have an order one phase in their mass matrix.

These conditions have interesting implications for the neutralino relic density in the MSSM. Furthermore, CP-violating phases are very strongly constrained by the experimental limit on the electric dipole moment of the electron. Many studies of both dark matter and electroweak baryogenesis within the MSSM have been made, but few of these considered both together. In ANL-HEP-PR-04-137 (hep-ph/0412264, to be published in Phys. Rev. D), we investigated these issues in a unified way. We find that both EWBG and neutralino dark matter can be explained simultaneously by the MSSM, but in a restricted region of parameters. This parameter region is expected to be probed in the near future by collider and dark matter direct detection experiments, and perhaps even sooner by attempts to measure the electric dipole moment of the electron.

From the standard unification relation for the gaugino mass parameters $\left(M_{2} \cong 2 M_{1}\right)$ follows that the lightest neutralino is dominated by the admixture of the $U(1)_{\Upsilon}$ gauge boson. Assuming that the lightest neutralino is the LSP, this implies a dark matter density, which is typically higher than required by the latest WMAP measurements. If, on the other hand, the lighter stop is sufficiently close to the LSP in mass, their coannihilation can reduce the dark matter density to comply with WMAP. The reduction can also happen by neutralinos resonantly annihilating via Higgs bosons in the early Universe. As a result, in the presence of a light stop and Higgs, the neutralino relic abundance can satisfy the WMAP data. This is illustrated by the horizontal green (medium gray) bands of Fig. 1. 

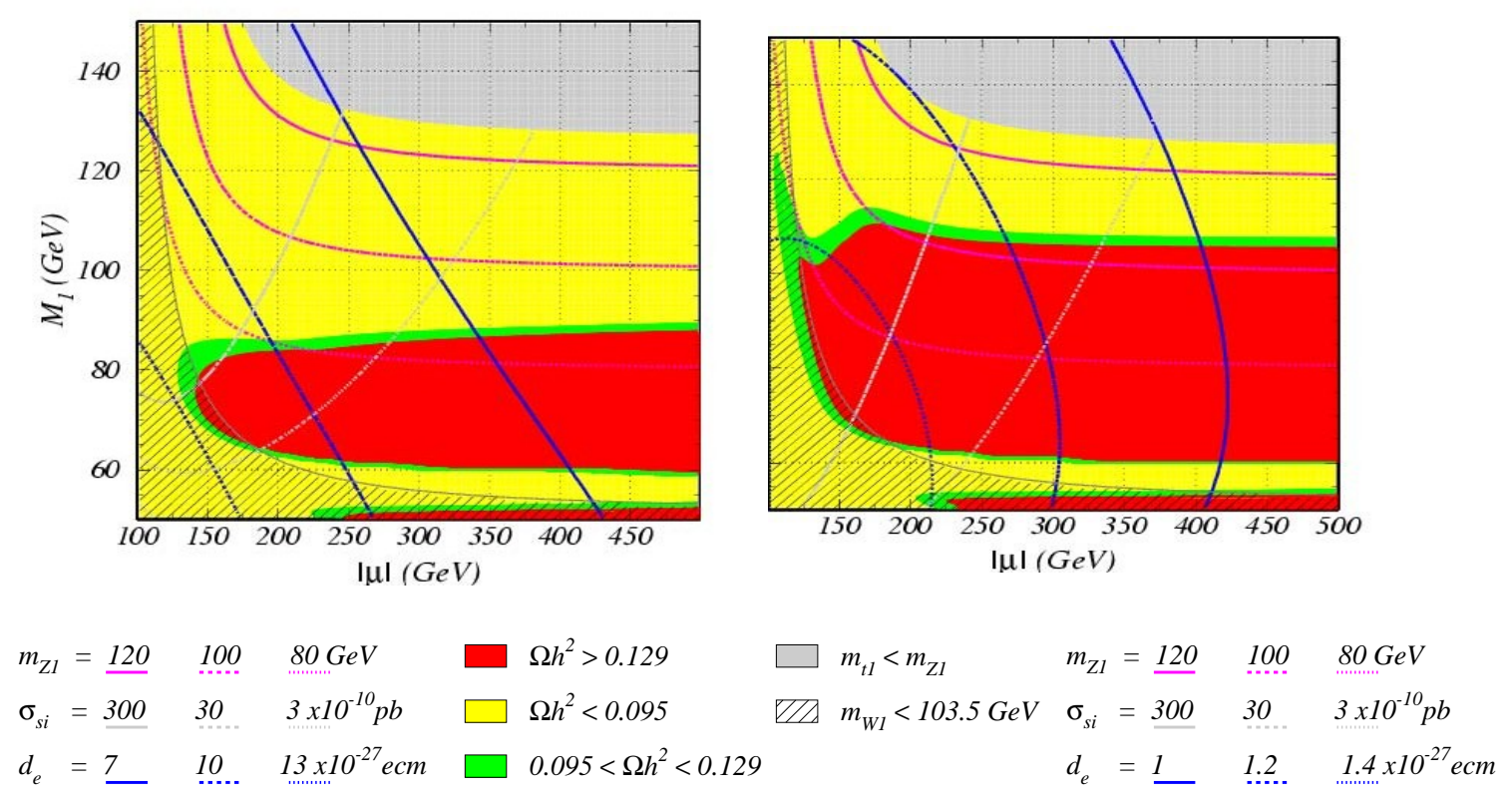

Figure 1. Neutralino relic density for $M_{A}=200 \mathrm{GeV}$ (left) and $M_{A}=1000 \mathrm{GeV}$ (right), and $\operatorname{Arg}(\mu)=\pi / 2$.

These green bands show the region of parameter space where the neutralino relic density is consistent with the $95 \%$ CL limits set by the WMAP data. The regions in which the relic density is above [below] the experimental bound and excluded by more than two standard deviations are indicated by the red (dark gray) [yellow (light gray)] areas. Finally, in the (medium-light) gray region at the upper right, the lightest stop becomes the LSP, while in the hatched area at the lower left corner, the mass of the lightest chargino is lower than is allowed by LEP data.

The $\mathrm{CP}$ violating phases needed for electroweak baryogenesis are very strongly constrained by the experimental limit on the electric dipole moment (EDM) of the electron. These phases must appear in the chargino mass matrix. In this case, the oneloop order contribution to the electron EDM involves intermediate scalar fermions of the first and second generations. If these scalar fermions are relatively heavy, $m_{\tilde{f}} \gtrsim 10 \mathrm{TeV}$, the one-loop contribution to the electron (and neutron) EDM becomes negligible, even for phases of order one. Such heavy masses have almost no effect on EWBG. At twoloop order, however, there are diagrams involving intermediate charginos and Higgs bosons, which cannot be decoupled if EWBG is to be successful. These contributions are reduced for larger values of the CP-odd Higgs mass parameter, $M_{a}$, and smaller values of $\tan \beta$, but are still sizeable. We find that EWBG is still viable, but that an order-ofmagnitude improvement of the electron EDM limit will come close to ruling out this mechanism within the MSSM.

(C. Wagner and C. Balazs) 


\section{II.A.12. Gauged Skyrmions and WZW Interactions in Dimensional Deconstruction}

Dimensional deconstruction is the intricate systematic accounting of correspondences of higher-dimensional gauge theories into $D=4$ matter-coupled gauge theories, including current models for electroweak interactions. The technique goes far beyond conventional Kaluza-Klein collective mapping of degrees of freedom and spacetime symmetries, and still has areas in need of specification. C. Hill and C. Zachos have published two papers on unexpected correspondence rules discovered in examining the consistency of covariant derivatives involving the lowest $\mathrm{K}-\mathrm{K}$ modes with the anomaly and topological structure of such theories.

A remarkable correspondence of this type is the devolution of $\mathrm{D}=5$ pure-gauge "instantonic monopoles" to $\mathrm{D}=4$ gauged skyrmions. The latter are topologically nontrivial solutions which feature in the 't Hooft baryon-number violating determinant, as well as models of effective vector-meson interactions with nucleons. Y. Brihaye, C. Hill, and C. Zachos discovered a notable phenomenon [Phys. Rev. D70, 111502 (2004)]: Gauged skyrmion masses are bounded above, i.e., their mass cannot exceed a fixed "monopolic" scale.

Normally, standard (ungauged) skyrmion masses are proportional to the "Skyrme term" coupling in the effective pseudoscalar chiral Lagrangian. This term is needed for stabilizing skyrmion cores against collapse. As a result, such masses can grow indefinitely with the Skyrme coupling, and so this coupling must be input arbitrarily to the value required by nucleons, begging the question of how the Skyrme term acquires its precise coefficient in effective QCD--and not a different one. In striking contrast, for gauged skyrmions, BHZ found that, for large Skyrme couplings, such couplings lose relevance, and do not influence skyrmion masses appreciably: These are thus limited in size to essentially monopole masses, $\mathrm{O}(\mathrm{v} / \mathrm{g})$. As a result, the particular coupling of the Skyrme term is not crucial. Consequently, its impact on baryogenesis may well be robust (free of sensitive dependence on such an elusive coupling). The mechanism discovered relies on a Born-Oppenheimer-type decoupling of the effective scales of the degrees of freedom of the problem, in which the balanced skyrmion variables freeze out, in turn containing the gauge variables within a characteristic radius. This radius depends on the Skyrme coupling only weakly, in stark contrast to the standard ungauged skyrmion case, where it is proportional to the coupling.

Hill and Zachos further discovered that deconstruction provides insight into another long-standing enigma, namely the connection between Chern-Simons topological interactions in odd-dimensional gauge theories and Wess-Zumino-Witten terms in even dimensional chiral models--terms required in effective QCD, but so far inaccessible to deconstruction methods. Specifically, in Phys. Rev. D71, 046002 (2005), they developed a new technique for the derivation of the Wess-Zumino-Witten terms of $\mathrm{D}=4$ gauged 
chiral systems from $\mathrm{D}=5$ pure (mesonless) Yang-Mills theory that includes relevant gauge-field Chern-Simons terms. In particular, they compactified the theory on an orbifold, to derive the effective $\mathrm{D}=4$ Lagrangian by structuring special lattice techniques consistent with the underlying anomalies. (The pseudoscalar mesons arise from the lattice Wilson links.) This yields the WZW term with the integral Witten coefficient, by way of a simple heuristic argument.

They further discovered a novel WZW term for singlet currents, which yields the full Goldstone-Wilczek current, and a U(1) axial current for the skyrmion, again with the appropriate anomaly structures. They finally presented a more detailed analysis of the dimensional descent of Yang-Mills in $D=5$ into a gauged chiral Lagrangian in $D=4$, heeding the consistency of the $\mathrm{D}=4$ and $\mathrm{D}=5$ Bianchi identities: These dictate a novel covariant derivative structure in the $\mathrm{D}=4$ gauge theory, yielding a field strength modified by the addition of commutators of chiral currents. The Chern-Simons term of the pure $\mathrm{D}=5$ Yang-Mills theory then devolves into the correct form of the Wess-Zumino-Witten term with an index (the analog of $N_{\text {colors }}=3$ ) of $\mathrm{N}=\mathrm{D}=5$. This theory also has a Skyrme term with a fixed coefficient, as well as a new gauged Skyrme term.

(C. Zachos) 


\section{ACCELERATOR RESEARCH AND DEVELOPMENT}

\section{III.A. ARGONNE WAKEFIELD ACCELERATOR PROGRAM}

\section{III.A.1. The Argonne Wakefield Accelerator Facility Status}

AWA Facility Status and Activities: The new $1-1 / 2$ cell RF photocathode gun has been in operation for the last two years. This gun has significantly improved the present AWA drive beam performance. Currently the gun produces beams with energy of $\sim 7 \mathrm{MeV}$ and single bunch charges up to $100 \mathrm{nC}$ with the $\mathrm{Mg}$ cathode installed. We measured bunch lengths of 10 ps FWHM for charges up to $70 \mathrm{nC}$. We implemented an optical system for 'laser cleaning' the $\mathrm{Mg}$ cathode with a focused laser beam. After laser cleaning, the quantum efficiency of the installed $\mathrm{Mg}$ cathode improved by a factor of 5 10. This allows us to begin the much-anticipated high-gradient and high-power tests without waiting for CsTe based cathode. These results show that the new AWA gun has successfully reached its design goal and that the beam parameters meet with the requirements of the planned two-beam acceleration experiments and other high current beam physics experiments.

Two linac structures were removed from the original AWA drive beamline and thoroughly cleaned, allowing them to be installed in the new AWA beamline without degradation of its ultra high vacuum (mid $10^{-10}$ Torr with RF off). One of these linac structures has already been installed and RF conditioned. The RF conditioning process took a considerable amount of time, likely due to the fact that the cleaning process altered the inner surfaces of the copper cells and the linac is now operating at a higher power level than before. Currently, the total beam energy has been conditioned up to $13.5 \mathrm{MeV}$ with goal of achieving $18 \mathrm{MeV}$. The cause of this lower beam energy is currently under investigation, but it appears to be due to some newly installed RF waveguides. We expect to fix this low energy condition soon; enabling us to conduct the high-gradient wakefield experiments.

A $15 \mathrm{GHz}$ standing-wave dielectric loaded wakefield structure has been recently fabricated and installed in the AWA beamline. In addition, a $6 \mathrm{GHz}$ oscilloscope and a newly developed RF mixer circuit were designed to analyze the time-domain envelope of the wakefields generated by the beam traversing the structure. Using a drive bunch charge of approximately $\sim 20 \mathrm{nC}$, we have begun initial characterization of the wakefield using a carefully designed RF mixer circuit. The initial results are every encouraging and agrees well with our predictions. Figure 1 and 2 show the experimental set-up and preliminary data. Our next step will be

An RF loop antenna is installed in the vacuum port of the AWA gun to characterize the RF performance of the gun. The use of a recently acquired high 
bandwidth oscilloscope allows a very detailed observation of the $1.3 \mathrm{GHz}$ loop antenna signal, showing the beam loading effect. Further measurements at higher bunch charges, with single bunch and bunch trains, is to provide invaluable insights into the physics of high charge bunch generation.

\section{Direct Wakefield Measurement}

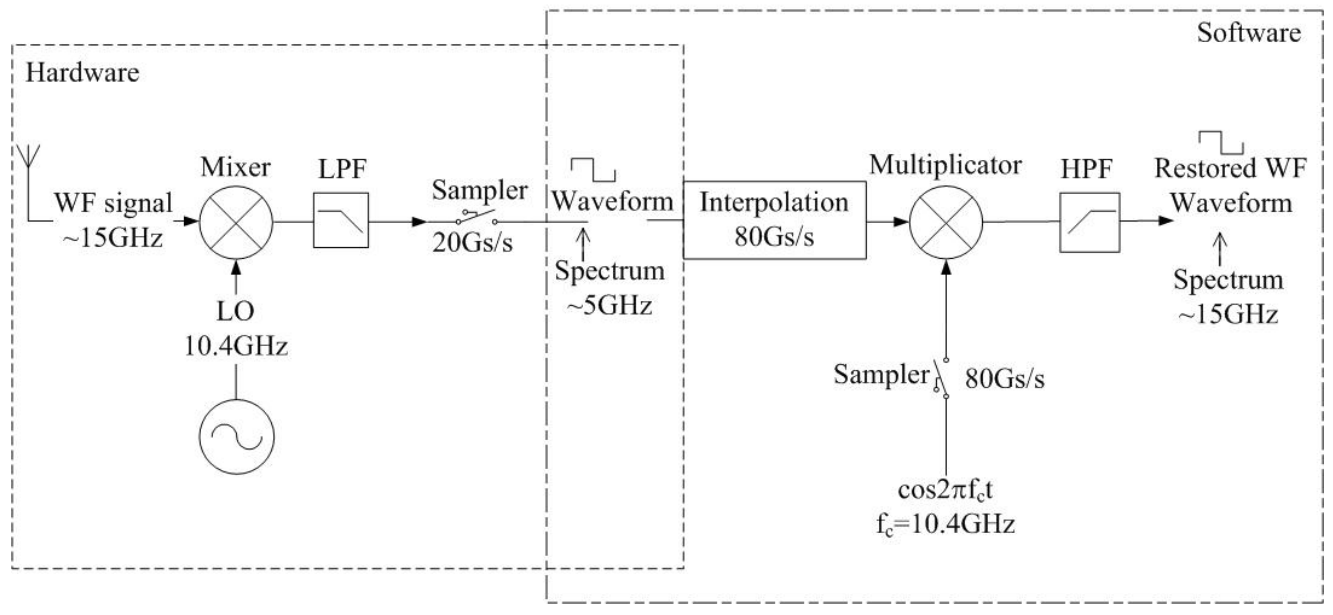

Figure 1. Block diagram of wakefield RF measurement technique.
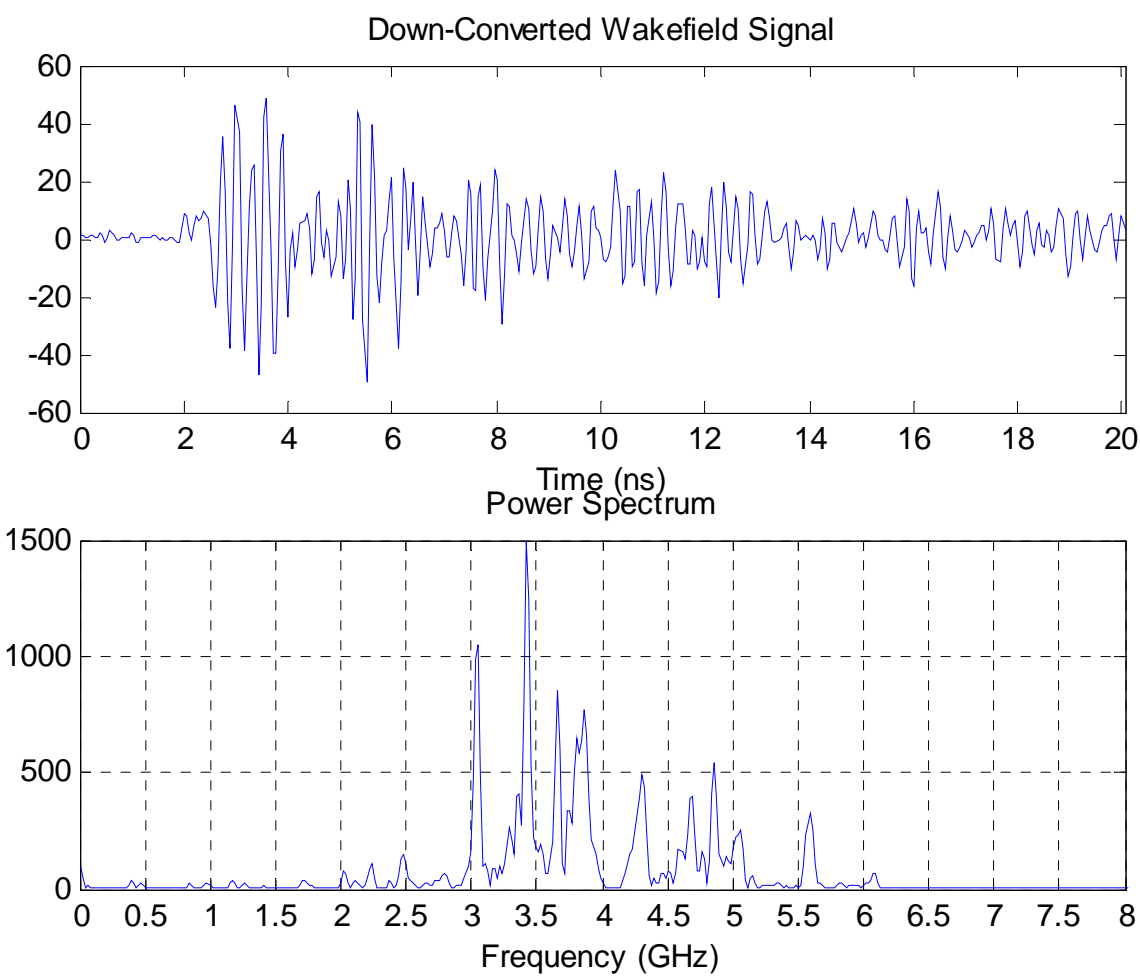

Figure 2 Down converted wakefield signal with the major frequency shown at $3.4 \mathrm{GHz}$. 
High $Q E$ cathode upgrade for the new $R F$ photocathode Drive gun and demonstration of high current beam generation. Based on the suggestions from the DOE review committee, we have initiated work on the construction of a high QE photocathode fabrication system. An ultra-high vacuum chamber and thermal vapor deposition system to fabricate $\mathrm{Cs}_{2} \mathrm{Te}$ photocathode is being assembled. The design of the system incorporates several considerations: (i) the need for a UHV transfer from the fabrication chamber to the photoinjector gun; (ii) the ability to fit into the current photoinjector gun at the AWA facility; (iii) the possibility of being adapted and modified to suit future photoinjector design and other types of photocathode fabrication. The deposition chamber is almost ready for initial testing. The vacuum chamber (donated by the Argonne Chemistry Division) has been thoroughly cleaned and is ready for the assembly of the various components: cathode support, heaters, deposition material holders, thermocouples, thickness monitors, and a UV arc-discharge lamp to be used for in-situ quantum efficiency measurements. It is expected that the first series of fabrication tests will begin soon.

Laboratory Astro-physics - measurements of fluorescent emission from air: Collaboration with a research group at the University of Chicago (Nobel prize winner Prof. Cronin) has been initiated, with the goal of making air fluorescence measurements using the AWA electron beam. These measurements have never been made in the range of electron beam energies accessible at the AWA facility (5 to $20 \mathrm{MeV}$ ), and are necessary for the calibration of instruments used to detect ultra-high energy cosmic rays entering Earth's atmosphere. In order to make these measurements, the AWA beamline was extended by about one meter to allow for the installation of a set of three quadrupoles at the downstream end. A thin Beryllium window was installed at the very end of the beamline, allowing the electron beam to leave the evacuated beamline and propagate in air. In the near future (Spring of 2005) the electron beam will enter a chamber with an adjustable Nitrogen pressure, where the fluorescence measurements will be conducted.

Positron source development at ANL. After the International Technology Recommendation Panel's decision on the technology choice for the International Linear Collider (ILC), with our group's expertise in high current and high brightness beam generations, we have started to investigate positron source production, accumulation and acceleration schemes for the ILC. The positron source in this study is based on gamma rays on a thin Ti target. A detailed PARMELA simulation has been done to study beam dynamic in positron capture optics, which consists of an Adiabatic Matching Device (AMD) and a copper pre-accelerator. Both positron and electron beam initial distributions are produced by using EGS4 from the target, and with beam energy $120 \mathrm{MeV}$ at the end of the pre-accelerator. By adjusting the injection phase and adiabatic matching solenoid, positron capture efficiency of $27 \%$ can be achieved with beam energy spread of $\pm 1 \%$ and emittance $\varepsilon x+\varepsilon y<0.048 \mathrm{~m} \cdot \mathrm{rad}$. We have also both electron and positron losses along the beam lines, thus the background radiations. 
Exotic Accelerator Physics. We have explored several exotic accelerator physics applications and continue to actively seek out new opportunities. In this way, we continue to increase our knowledge base in the most exotic and advanced areas of accelerator physics including Photonic Band Gap (PBG) materials and Left-Handed Metamaterials (LHM). During the last year, we have investigated three novel accelerator physics topics: (1) The Multilayer Dielectric-Loaded Accelerator (multilayer DLA); (2) The Reverse Cherenkov Radiator (RCR); and (3) The Left-handed Metamaterial Accelerator (LHM accelerator). The first of these devices is an example of a 1D PBG structure since it prohibits propagation in the radial direction. It is similar to our standard single-layer DLA, but with vastly improved power attenuation. This work resulted in a publication in NIM. We also investigated two ways to make use of the recently discovered LHM. In the first scheme, the RCR, we have designed a cherenkov radiator where cherenkov radiation propagates in the direction opposite to the electron beam. We have simulated this device using Microwave Studio $\rightarrow$ and have now begun to make cold test measurements with an HP Network Analyzer. Based on the knowledge and expertise gained with the RCR, we are also beginning to design an accelerating structure based on LHM in collaboration with Prof. G. Shvets of UT at Austin.

We also carried out a novel OTR interferometry (OTRI) experiment (in collaboration with R. Fiorito of U.M.D.) that, in addition to measuring the usual rms emittance, should also be capable of measuring the energy spread. This technique is necessary when measuring the emittance directly from a photoinjector due to the nonnegligible energy spread. This technique is based on measuring the effects of all the parameters effecting the visibility of OTR interference fringes, such as energy spread, angular divergence, the ratio of foil separation to wavelength ratio, $\mathrm{d} / \lambda$ and the filter bandpass. The initial experiments were carried out in the Fall 2004, and subsequent

analysis showed that a different foil spacing is needed for the AWA setup. Further experiments are planned for 2005.

\section{III.A.2. Development of externally-driven, $11.424 \mathrm{GHz}$, dielectric-loaded structures and high-power tests at NRL}

This is an exciting near term application of wakefield derived technology using an external rf power source to drive a dielectric structure. A series of high power tests were conducted at NRL during this period, and a number of significant physics results were obtained.

To further test the dielectric joint breakdown, we recently filled the gap at the joint with a high-Q epoxy and performed a high-power test. According to the manufacturer, the epoxy has a dielectric constant of 5.2 and a dielectric strength of 23.6 $\mathrm{MV} / \mathrm{m}$. Based on this dielectric constant, we expect that the local field enhancement factor should be reduced from 20 to 3.8 thus allowing higher gradient operation. As 
expected, the accelerating field realized during this high-power test increased to 7.5 $\mathrm{MV} / \mathrm{m}$ (from $5.7 \mathrm{MV} / \mathrm{m}$ ) before the typical breakdown signatures were observed. This accelerating electric field implies an electric field of $\sim 28 \mathrm{MV} / \mathrm{m}$ at the upstream dielectric joint. This field is greater than the dielectric strength of the epoxy which is why breakdown occurred. The conclusion we draw from the high-power RF tests of the MCT based DLA structure is that all the gaps must be avoided. Therefore, we have started to develop a new structure design to test a gapless dielectric loaded accelerating structure in the near future.

(W. Gai)

\section{B. MUON COLLIDER RF R\&D}

With the MTA not operational yet, the primary effort was in looking at old data, preparing papers and designing the Atom Probe Tomography system that will help us understand the breakdown process experimentally.

There were two European conferences during this period. At the European Particle Accelerator Conf, EPAC'04, in Lucerne, we presented a shortened version of our model for triggers in rf breakdown. At the Linear Accelerator Conference, LINAC04, we presented a summary of the data at our High Gradient RF workshop in Sept '03. Two papers were also published, one in NIM (Norem, Insepov and Konkashbaev) describing the overall trigger model, and one in Phys. Rev. STAB, (Insepov, Norem and Hassanein) describing a theoretical model of the breakdown trigger mechanism.

\section{High Gradient RF studies}

One of the primary conclusions of the work we have been doing on $\mathrm{rf}$ breakdown is that the pre-breakdown environment we measure in rf cavities ( 0.1 micorn asperities, with electric fields on the order of $10 \mathrm{GV} / \mathrm{m}$ ) has been studied for many years in Atom Probe Tomography, and the exact measurements we would like to make have been made for years in this environment. We have since begun to collaborate with the David Seidman group at Northwestern, who are some of the world leaders in this work.

The primary experimental effort was to hire Dr. Jason Sebastian into the Northwestern University Atom Probe Tomography Group, first for a short term and then more permanently. We then proceeded to design a system for applying and measuring coatings, as part of an Atom Probe Tomography system. This system will enable us to clean or prepare samples in a variety of ways, add coatings, measure the field emission coefficient under realistic conditions, and then remove the coating atom by atom to measure the sticking coefficient. The ultimate aim of this work would be to produce

surfaces that did not produce dark current beams and were not subject to increased breakdown rates in static magnetic fields. 
We ordered parts for this system and began assembly of the evaporation chamber to be fitted on the Pulsed Laser Atom Probe. The assembly is proceeding and should be done around the middle of February.

The Atom Probe Tomography method produced very precise and detailed pictures of atomic positions within alloys and surfaces. The method uses field evaporation to remove and identify the positions and structure of individual atoms, and permits high statistics reconstruction of a material inside the computer. Atoms are identified by time of flight, and the position is recorded using a microchannel plate with
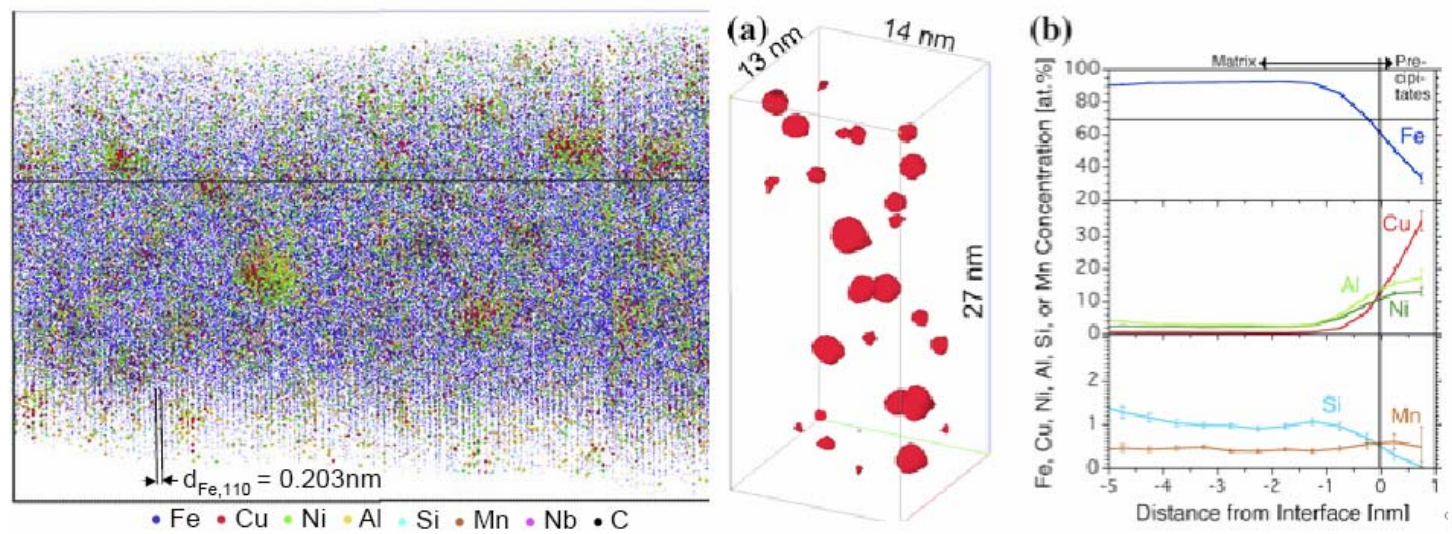

Figure 1. Different ways of looking at alloys using Atom Probe Tomography. Atomic planes, (as well as individual atoms) are easily seen.

$(x, y)$ readout. One of the problems with superconducting $\mathrm{rf}$ (SCRF) is that the supercurrents share the top $50 \mathrm{~nm}$ of the material with any oxides and impurities that may be present, making them very sensitive to their environment. The Northwestern group has the equipment and software that let them do more precise measurements of atomic structure than other methods. Thus, this method is attracting interest in the SCRF community for the most precise measurements of oxides, interstitial oxygen and hydrogen and the structure of grain boundaries, which are a problem in SCRF manufacturing. An example of the data is shown below. We (ANL, FNAL. NW) have submitted an abstract for the May PAC conference as an opportunity to present the first data from this work.

\section{Muon Test Area Program}

Problems with $805 \mathrm{MHz}$ klystrons turned off the Lab G experimental program in Dec 2003, and delays, primarily due to the Tevataron shutdown in Sept 2004, have slowed down the installation of the 805 and $201 \mathrm{MHz}$ cavities in the Muon Test Area, 
(MTA). At present the solenoid magnet and most of the waveguide and coax has been installed, and we anticipate being fully operational in March, 2003. A preliminary study of the field dependence of field emitters has led to a way of measuring enhancement factors for individual emitters, which we will use with MTA data.

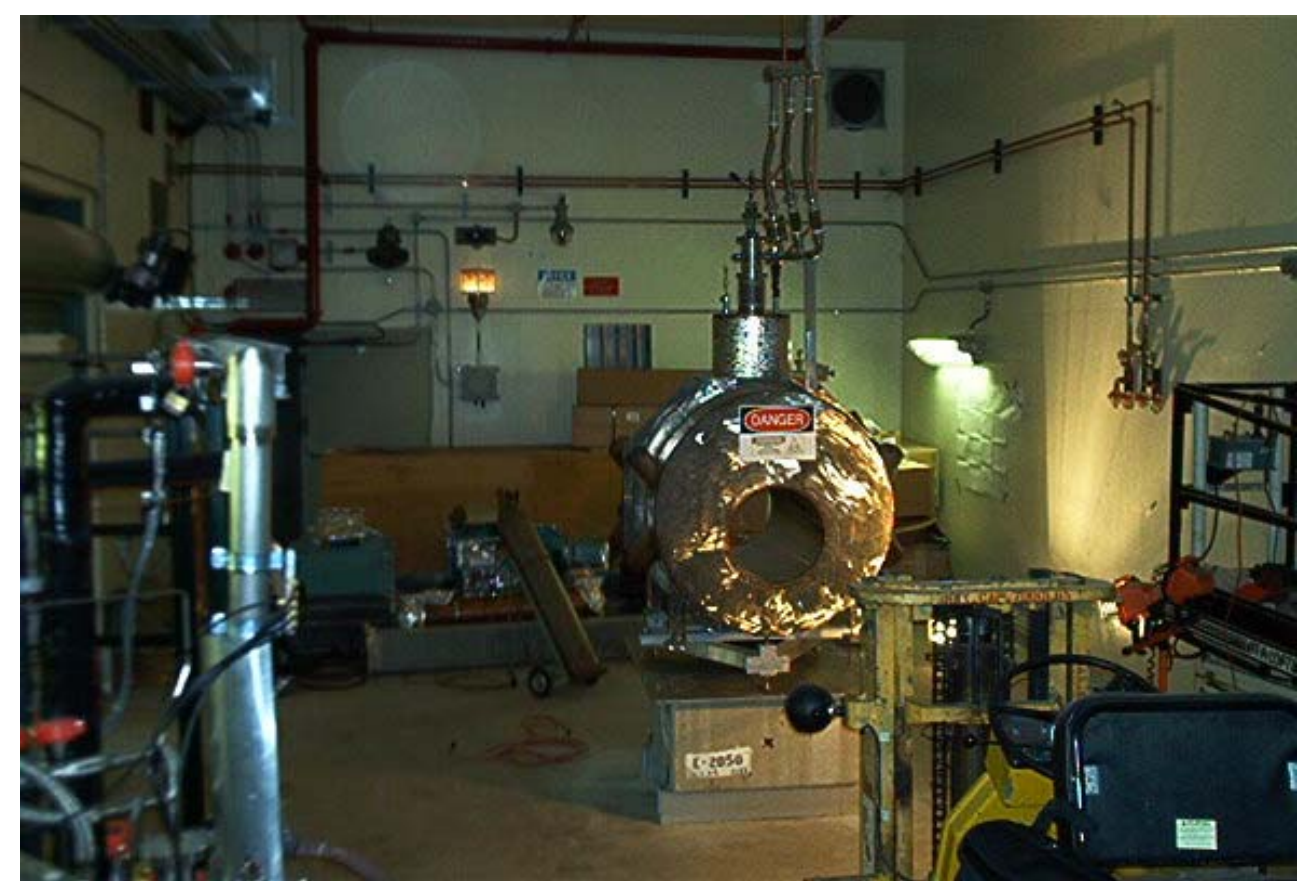

Figure 2: The Muon Test area, with solenoid magnet installed.

(J. Norem) 


\section{PUBLICATIONS}

\section{A. Books, Journals And Conference Proceedings}

A Comparison of Predictions for SM Higgs Boson Production at the LHC

C. Balazs, M. Grazzini, J. Huston, A. Kulesza, and I. Puljak

In: Les Houches 2003 "Physics at TeV Colliders" The Quantum

Chromodynamics and the Standard Model Working Group: Summary Report (Vol. 1), edited by B. Allanach (July 2004) pp. 51.

A Mass Inequality for the $\Xi^{*}$ and $\Theta^{+}$Pentaquarks

M. Karliner and H. J. Lipkin

Phys. Lett. B594, 273-276 (August 2004).

Advanced Grid Technologies in ATLAS Data Management

A. Vaniachine

In: Proceedings of the XIX International Symposium of Nuclear Electronics

and Computing (NEC 2003), ed. by (JINR, Dubna, August 2004) pp. 273-280.

Azimuthal Anisotropy and Correlations at Large Transverse Momenta in $\mathrm{p}+\mathrm{p}$ and $\mathrm{Au}+\mathrm{Au}$ Collisions at $\sqrt{s}_{\mathrm{NN}}=200 \mathrm{GeV}$

R.V. Cadman, H.M. Spinka and D.G. Underwood

Phys. Rev. Lett. 93, 252301 (December 2004).

ANL-HEP-PR-04-120

Bounding Gauged Skyrmion Masses

Y. Brihaye, C. T. Hill, and C. K. Zachos

Phys. Rev. D70, 111502 (R) (December 2004).

Branes, Quantum Nambu Brackets, and the Hydrogen Atom

C. Zachos and T. Curtright

Czech. J. Phys. 54 (Springer, November 2004) pp. 1393-1398.

Branes, Strings, and Odd Quantum Nambu Brackets

T. L. Curtright and C. K. Zachos

In: "Quantum Theory and Symmetries", Proceedings of the $3^{\text {rd }}$ International

Symposium on Quantum Theory and Symmetries (QTS3), edited by

P. C. Argyres, et al. (World Scientific, London, October 2004) pp. 206-217.

$B_{S}-\bar{B}_{S}$ Mixing in $Z^{\prime}$ Models with Flavor-Changing Neutral Currents

V. Barger, C.-W. Chiang, J. Jiang, and P. Langacker

Phys. Lett. B596, 229-239 (August 2004). 
Centrality and Pseudorapidity Dependence of Charged Hadron Production at Intermediate $\mathrm{p}_{\mathrm{T}}$ in $\mathrm{Au}+\mathrm{Au}$ Collisions at $\sqrt{s}_{\mathrm{NN}}=130 \mathrm{GeV}$

R. V. Cadman, K. Krueger, H. M. Spinka and D. G. Underwood

Phys. Rev. C70, 044901 (October 2004).

ANL-HEP-PR-04-53

Dark Matter, Light Stops and Electroweak Baryogenesis

C. Balazs, M. Carena, and C.E.M. Wagner

Phys. Rev. D70, 015007 (July 2004).

Determining the Extra-Dimensional Location of the Higgs Boson

A. Aranda, C. Balazs, J. L. Diaz-Cruz, S. Gascon-Shotkin, and O. Ravar

In: Les Houches 2003, "Physics at TeV Colliders", Beyond the Standard Model

Working Group: Summary Report (Vol. 3), ed. by B. C. Allanach (July 2004) pp. 92.

Differential Cross Section of the Charge-Exchange Reaction $\pi^{-} p \rightarrow \pi^{\circ} n$ in the Momentum Range from 148 to $323 \mathrm{MeV} / \mathrm{c}$

C.E. Allgower and H.M. Spinka

Phys. Rev. C69, 055206 (May 2004). not previously cited

Electroweak Baryogenesis and Dark Matter in the nMSSM

A. Menon, D. E. Morrissey, and C.E.M. Wagner

Phys. Rev. D70, 035005 (August 2004).

Exclusive Two Charmonium Versus Charmonium Glueball Production at Belle Jungil Lee

In: J. Korean Phys. Soc. (Vol. 45, Suppl.), ed. by E. J. Chun (Korean Phys. Soc., Seoul, November 2004) pp. S354-S359.

Hadronic Final States

S. Chekanov, M. Dasgupta, D. Milstead et al

In: Proc. of the DIS04 workshop, CERN-PH-TH/2004-184, hep-ph/0409284,

Slovakia, Tatri, IEP SAS 2004, Kositce, pp. 38, (April 2004). not previously cited

Hard Probes in Heavy Ion Collisions at the LHC: Heavy Flavor Physics

G. T. Bodwin, J. Lee, and R. Vogt

In: CERN Yellow Book "Hard Probes in Heavy Ion Collisions at the LHC", edited by M. Mangano, et al. (CERN, Geneva, October 2004) pp. 265-277.

Heavy Flavor in $W+$ Jets Production at the Fermilab Tevatron

J. M. Campbell and J. Huston

Phys. Rev. D70, 094021 (November 2004). 
Higgs Boson and Diphoton Production at the LHC

P. Skands, B. C. Allanach, H. Baer, C. Balazs, et al.

In: Les Houches 2003, "Physics at TeV Colliders", Higgs Working Group:

Summary Report (Vol. 2), edited by M. Spira (July 2004) pp. 48.

Higgs Boson Production in Weak Boson Fusion at Next-to-Leading Order

E. L. Berger and J. M. Campbell

Phys. Rev. D70, 073011 (October 2004).

Inclusive Quarkonium Production and the NRQCD-Factorization Approach

G. T. Bodwin

In: J. Korean Phys. Soc. (Vol. 45, Suppl.), ed. by E. J. Chun (Korean Phys.

Soc., Seoul, November 2004) pp. S306-S312.

$K^{-} p \rightarrow \pi^{\circ} \pi^{\circ} \Sigma^{\circ}$ at $p_{K_{-}}=514-750 \mathrm{MeV} / \mathrm{c}$ and Comparison with Other $\pi^{\circ} \pi^{\circ}$ Production .

C.E. Allgower and H.M. Spinka

Phys. Rev. C70, 034605 1-4 (September 2004).

Measurement of the $t \bar{t}$ Production Cross Section in $p \bar{p}$ Collisions at $\sqrt{s}=1.96 \mathrm{TeV}$ Using Dilepton Events

W. Ashmanskas, R. E. Blair, K. L. Byrum, E. Kovacs, S. E. Kuhlmann, T. LeCompte,

L. Nodulman, J. Proudfoot, M. Tanaka, R. Thurman-Keup, R. G. Wagner and A. B. Wicklund Phys. Rev. Lett. 93, 142001 (October 2004).

Measurement of Inverse Pion Photoproduction at Energies Spanning the N(1440) Resonance

C.E. Allgower and H.M. Spinka

Phys. Rev. C70, 035204 (September 2004).

Measurement of Partial Widths and Search for Direct CP Violation in D0 Meson Decays to $\mathrm{K}-\mathrm{K}+$ and $\mathrm{p}-\mathrm{p}+$

W. Ashmanskas, R. E. Blair, K. L. Byrum, E. Kovacs, S. E. Kuhlmann, T. LeCompte,

L. Nodulman, J. Proudfoot, M. Tanaka, R. Thurman-Keup, R. G. Wagner and A. B. Wicklund

Phys. Rev. Lett. 94, 041803 (August 2004).

Measurements of Transverse Energy Distributions in Au+Au Collisions at $\sqrt{s}=200 \mathrm{GeV}$

R.V. Cadman, H.M. Spinka and D.G. Underwood

Phys. Rev. C70, 054907 (November 2004).

ANL-HEP-PR-04-121 
Nambu Dynamics, Deformation Quantization, and Superintegrability

T. L. Curtright and C. K. Zachos

CRM Proceedings and Lecture Notes of the Workshop on Superintegrability in Classical and Quantum Systems, Vol. 37, ed. by P. Tempesta et al., (AMS, Providence, RI, December 2004) pp. 29-46.

New Mechanism of Cluster-Field Evaporation in rf Breakdown J.H. Norem, A. Hassanein and Z. Insepov

Phys. Rev. Special Topics - Accelerators and Beams 7, 122001 (December 2004).

Photon and Neutral Pion Production in $\mathrm{Au}+\mathrm{Au}$ Collisions at $\sqrt{s}_{\mathrm{NN}}=130 \mathrm{GeV}$

R. V. Cadman, K. Krueger, H. M. Spinka and D. G. Underwood

Phys. Rev. C70, 044902 (October 2004).

ANL-HEP-PR-04-24

Production of e+e- Pairs Accompanied by Nuclear Dissociation in Ultra-Peripheral Heavy Ion Collisons

R. V. Cadman, K. Krueger, C.J. McClain, H. M. Spinka and D. G. Underwood Phys. Rev. C70, 031902 (R), (September 2004).

Properties and Performance of the Prototype Instrument for the Pierre Auger Observatory H.M. Spinka

Nuclear Instruments \& Methods in Physics Research - Elsevier 523, pp. 50-95, (May 2004). not previously cited

Pseudorapidity Asymmetry and Centrality Dependence of Charged Hadron Spectra in $\mathrm{d}+\mathrm{Au}$ Collisions at $\sqrt{s}_{\mathrm{NN}}=200 \mathrm{GeV}$

R.V. Cadman, H.M. Spinka and D.G. Underwood

Phys. Rev. C70, 064907 (December 2004).

ANL-HEP-PR-04-119

Reaction $K^{-} p \rightarrow \pi^{0} \pi^{0} \Lambda$ from $p_{K-}=514$ to $750 \mathrm{MeV} / \mathrm{c}$

C.E. Allgower and H.M. Spinka

Phys. Rev. C69, 042202 (April 2004). not previously cited

Search for Doubly-Charged Higgs Bosons Decaying to Dileptons in $p \bar{p}$ Collisions at $\sqrt{s}=1.96 \mathrm{TeV}$

W. Ashmanskas, R. E. Blair, K. L. Byrum, E. Kovacs, S. E. Kuhlmann, T. LeCompte, L. Nodulman, J. Proudfoot, M. Tanaka, R. Thurman-Keup, R. G. Wagner and A. B. Wicklund Phys. Rev. Lett. 93, 221802 (June 2004). not previously cited 
Single Top Production and Decay at Next-to-Leading Order

J. M. Campbell, R. K. Ellis, and F. Tramontano

Phys. Rev. D70, 094012 (November 2004).

Schottky-Enabled Photoemission in a RF Accelerator Photoinjector - Possible Generation of Ultralow Trasverse Thermal Emittance Electron Beam

Z. Yusof, M.E. Conde and W. Gai

Phys. Rev. Letts 93, 114801 (September 2004).

Summary of the Argonne Workshop on High Gradient RF

J. Norem

Published in Proceedings of LINAC 2004, Lubeck, Germany, August 2004, pp. 601.

Supersymmetric SO(10) Models Inspired by Deconstruction

C.-S. Huang, J. Jiang, and T.-J. Li

Nucl. Phys. B702, 109-130 (December 2004).

SUSY Les Houches Accord: Interfacing SUSY Spectrum Calculators, Decay Packages, and Event Generators

P. Skands, B. C. Allanach, H. Baer, C. Balazs, et al.

JHEP 0407:036 (July 2004).

The Sensitivity of the LHC for TeV Scale Dimensions in Dijet Production

C. Balazs, M. Escalier, S. Ferrag, B. Laforge, and G. Polesello

In: Les Houches 2003, "Physics at TeV Colliders", Beyond the Standard Model

Working Group: Summary Report (Vol. 3), ed. by B. C. Allanach (July 2004), pp. 95.

The Argonne Wakefield Accelerator Facility Capabilities and Experiments

M. Conde, S. Antipov, W. Gai, C. Jing, R. Konecny, W. Liu, J.G. Power, H. Wang and

Z. Yusof

Published in Proceedings for AAC 2004, AIP Conference Proceedings (Stony Brook, NY, 2004) 737, pp. 657.

ANL-HEP-CP-04-74

Triggers for RF breakdown

J. Norem and Z. Insepav

Published in 9th European Particle Accelerator Conference (EPAC'04) (CERN, DESY), Lucerence, Switzerland (2004), pp. 1063.

ANL-HEP-CP-04-68 
Warped Unification, Proton Stability and Dark Matter

K. Agashe and G. Servant

Phys. Rev. Lett. 93, 231805 (December 2004).

Why the $\Theta^{+}$is Seen in Some Experiments and Not in Others-A Possible Explanation

M. Karliner and H. J. Lipkin

Phys. Lett. B597, 309-313 (September 2004).

y-Scaling in Quasifree Pion-Single-Charge Exchange

H.M. Spinka

Phys. Rev. C69, 064612, June (2004). not previously cited

ANL-HEP-PR-04-55

\section{IV.B. Major Articles Submitted For Publication}

A Step towards a Computing Grid for the LHC Experiments: ATLAS Data Challenge 1

ATLAS Collaboration, J. Gieraltowski, D. Malon, E. May, T. LeCompte,

A. Vaniachine et al.

Nucl. Instrum. \& Methods

ANL-HEP-PR-04-86

Analysis of Decay-Time Dependence of Angular Distributions in $\mathrm{B}_{-} \mathrm{s}^{\wedge} 0->\mathrm{J} / \mathrm{y} \mathrm{f}$ and B_d $d^{\wedge} 0->\mathrm{J} / \mathrm{y} \mathrm{K}{ }^{\wedge *} 0$ Decays and a Measurement of the Lifetime Difference between $B_{s}$ Mass

Eigenstates

R. E. Blair, K. L. Byrum, J. M. Cranshaw, S. E. Kuhlmann, T. LeCompte,

L. Nodulman, J. Proudfoot, M. Tanaka, R. G. Wagner and A. B. Wicklund Phys. Rev. Lett.

ANL-HEP-PR-04-144

Azimuthal Anisotropy in $\mathrm{Au}+\mathrm{Au}$ Collisions at $\sqrt{s}_{\mathrm{NN}}=200 \mathrm{GeV}$

R.V. Cadman, H.M. Spinka and D.G. Underwood

Phys. Rev. C.

ANL-HEP-PR-04-126

Baryon Number in Warped GUTs: Model Building and (Dark Matter Related) Phenomenology K. Agashe and G. Servant

JHEP

ANL-HEP-PR-04-18 
Bounding Gauged Skyrmion Masses

Y. Brihaye, C. T. Hill, and C. K. Zachos

Phys. Rev. D

ANL-HEP-PR-04-89

Comparison of Three-jet Events in $p \bar{p}$ Collisions at $\sqrt{s}=1.8 \mathrm{TeV}$ to Predictions from a Nextto-leading Order QCD Calculation

W. Ashmanskas, R. E. Blair, K. L. Byrum, E. Kovacs, S. E. Kuhlmann, T. LeCompte, L.

Nodulman, J. Proudfoot, M. Tanaka, R. Thurman-Keup, R. G. Wagner and A. B. Wicklund Phys. Rev. D.

ANL-HEP-PR-04-99

Dimensional Deconstruction and Wess-Zumino-Witten Terms

C. T. Hill and C. K. Zachos

Phys. Rev. D

ANL-HEP-PR-04-129

Electroweak Baryogenesis and New TeV Fermions

M. Carena, A. Megevand, M. Quiros and C.E.M. Wagner

Nucl. Phys. B

ANL-HEP-PR-04-87

First-Order Electroweak Phase Transition in the Standard Model with a Low Cutoff

C. Grojean, G. Servant, and J. D. Wells

Phys. Rev. Lett.

ANL-HEP-PR-04-63

High Power RF Test on X-Band Dielectric-Loaded Accelerating Structures

C. Jing, W.M. Liu, W. Gai and J.G. Power

IEEE Transaction on Plasma Science (IEEE)

ANL-HEP-PR-04-94

Measurement of Charged Particle Multiplicities in Gluon and Quark Jets in $p \bar{p}$ Collisions at $\sqrt{s}=1.8 \mathrm{TeV}$

W. Ashmanskas, R. E. Blair, K. L. Byrum, E. Kovacs, S. E. Kuhlmann,

T. LeCompte, L. Nodulman, J. Proudfoot, M. Tanaka, R. Thurman-Keup,

R. G. Wagner and A. B. Wicklund

Phys. Rev. Lett.

ANL-HEP-PR-04-77 
Measurement of the Forward-Backward Charge Asymmetry of Electron-Positron Pairs in $p \bar{p}$ Collisions at $\sqrt{s}=1.96 \mathrm{TeV}$

W. Ashmanskas, R. E. Blair, K. L. Byrum, J. M. Cranshaw, S. E. Kuhlmann, T.

LeCompte, L. Nodulman, J. Proudfoot, M. Tanaka, R. G. Wagner and A. B. Wicklund Phys. Rev. D

ANL-HEP-PR-04-135

Measurement of the $J / \psi$ Meson and b-Hadron Production Cross Sections in $p \bar{p}$ Collisions at $\sqrt{s}=1960 \mathrm{GeV}$

R. E. Blair, K. L. Byrum, J. M. Cranshaw, S. E. Kuhlmann, T. LeCompte, L.

Nodulman, J. Proudfoot, M. Tanaka, R. G. Wagner, A. B. Wicklund

Phys. Rev. D

ANL-HEP-PR-04-142

Measurement of the $t \bar{t}$ Production Cross Section in $p \bar{p}$ Collisions at $\sqrt{s}=1.96 \mathrm{TeV}$ Using Lepton + Jets Events with Secondary Vertex b-Tagging

W. Ashmanskas, R. E. Blair, K. L. Byrum, E. Kovacs, S. E. Kuhlmann, T. LeCompte, L. Nodulman, J. Proudfoot, M. Tanaka, R. Thurman-Keup, R. G. Wagner, A. B. Wicklund Phys. Rev. D

ANL-HEP-PR-04-132

Measurement of the $t \bar{t}$ Production Cross Section in $p \bar{p}$ Collisions at $\sqrt{s}=1.96 \mathrm{TeV}$ Using Kinematic Fitting of $b$-tagged Lepton + Jet Events

W. Ashmanskas, R. E. Blair, K. L. Byrum, E. Kovacs, S. E. Kuhlmann, T. LeCompte, L.

Nodulman, J. Proudfoot, M. Tanaka, R. Thurman-Keup, R. G. Wagner, A. B. Wicklund Phys. Rev. D

ANL-HEP-PR-04-98

Measurement of the $W$ Boson Polarization in Top Decay at CDF at $\sqrt{s}=1.8 \mathrm{TeV}$

R. E. Blair, K. L. Byrum, J. M. Cranshaw, S. E. Kuhlmann, T. LeCompte,

L. Nodulman, J. Proudfoot, M. Tanaka, R. G. Wagner, A. B. Wicklund

Phys. Rev. D

ANL-HEP-PR-04-143

Measurement of $\mathrm{Wg}$ and $\mathrm{Zg}$ Production in $p \bar{p}$ Collisions at $\sqrt{s}=1.96 \mathrm{TeV}$

W. Ashmanskas, R. E. Blair, K. L. Byrum, E. Kovacs, S. E. Kuhlmann, T. LeCompte,

L. Nodulman, J. Proudfoot, M. Tanaka, R. Thurman-Keup, R. G. Wagner and A. B. Wicklund Phys. Rev. Lett. (2004).

ANL-HEP-PR-04-100 
Method 2 at NLO

J. M. Campbell and J. Huston

Phys. Rev. D

ANL-HEP-PR-04-58

Minijet Deformation and Charge-Independent Two-Particle Correlations on Momentum

Subspace (h, f) in Au-Au Collisions at $\sqrt{s}_{\mathrm{NN}}=130 \mathrm{GeV}$

R.V. Cadman, H.M. Spinka and D.G. Underwood

Phys. Rev. Lett.

ANL-HEP-PR-04-127

Mode Analysis of a Multilayered Dielectric-Loaded Accelerating Structure

C. Jing, W.M. Liu, W. Gai and J.G. Power

Nuclear Instruments and Methods in Physics Research Section A: Accelerators, Spectrometers, Detector, and Associated

ANL-HEP-PR-04-93

On a Possible Tetraquark Cousin of the $\theta^{+}$

M. Karliner and H. J. Lipkin

Phys. Letts.

ANL-HEP-PR-04-113

Open Charm Yields in $\mathrm{d}+\mathrm{Au}$ Collisions at $\sqrt{s}_{\mathrm{NN}}=200 \mathrm{GeV}$

R.V. Cadman, H.M. Spinka and D.G. Underwood

Phys. Rev. Lett.

ANL-HEP-PR-04-125

Perturbative, Non-Supersymmetric Completions of The Little Higgs

P. Batra and D. E. Kaplan

JHEP

ANL-HEP-PR-04-139

Physics Interplay of the LHC and the ILC

E. L. Berger, J. Jiang, and C.E.M. Wagner, et al.

Phys. Reports

ANL-HEP-PR-04-108

RunMC - An Object-Oriented Analysis Framework for Monte Carlo Simulation of HighEnergy Particle Collisions

S. Chekanov

Comp. Phys. Comm. Journal

ANL-HEP-PR-04-112 
Search for Anomalous Kinematics in $t \bar{t}$ Dilepton Events at CDF II

R. E. Blair, K. L. Byrum, J. M. Cranshaw, S. E. Kuhlmann, T. LeCompte,

L. Nodulman, J. Proudfoot, M. Tanaka, R. G. Wagner and A. B. Wicklund Phys. Rev. Lett.

ANL-HEP-PR-04-146

Search for Electroweak Single Top Quark Production in $p \bar{p}$ collisions at $\sqrt{s}=1.96 \mathrm{TeV}$

R. E. Blair, K. L. Byrum, J. M. Cranshaw, S. E. Kuhlmann, T. LeCompte,

L. Nodulman, J. Proudfoot, M. Tanaka, R. G. Wagner and A. B. Wicklund Phys. Rev. Lett.

ANL-HEP-PR-04-148

Search for Excited and Exotic Electrons in the e $\gamma$ Decay Channel in $p \bar{p}$ Collisions at $\sqrt{s}=1.96 \mathrm{TeV}$

R. E. Blair, K. L. Byrum, J. Cranshaw, S. E. Kuhlmann, T. LeCompte, L. Nodulman, J. Proudfoot, M. Tanaka, R. G. Wagner and A. B. Wicklund

Phys. Rev. Lett.

ANL-HEP-PR-04-136

Search for Kaluza-Klein Graviton Emission in $p \bar{p}$ Collisions at $\sqrt{s}=1.8 \mathrm{TeV}$ Using the Missing Energy Signature

W. Ashmanskas, R. E. Blair, K. L. Byrum, E. Kovacs, S. E. Kuhlmann, T. LeCompte,

L. Nodulman, J. Proudfoot, R. Thurman-Keup, R. G. Wagner and A. B. Wicklund Phys. Rev. Letts

ANL-HEP-PR-03-92

Search for Narrow Charmed Baryonic State Decaying into $\mathrm{D}^{\wedge *+/-\mathrm{p}^{\wedge}-/+}$ in ep Collisions at HERA

S. Chekanov

Eur. J. Phys.

ANL-HEP-PR-04-106

Search for Scalar Leptoquark Pairs Decaying to $v \bar{v} q \bar{q}$ in $p \bar{p}$ Collisions at $\sqrt{s}=1.96 \mathrm{TeV}$

W. Ashmanskas, R. E. Blair, K. L. Byrum, J. M. Cranshaw, S. E. Kuhlmann, T.

LeCompte, L. Nodulman, J. Proudfoot, M. Tanaka, R. G. Wagner and A. B. Wicklund Phys. Rev. D.

ANL-HEP-PR-04-134 
Single To Production and Decay at Next-to-Leading Order

J. M. Campbell, R. K. Ellis, and F. Tramontano

Phys. Rev. D

ANL-HEP-PR-04-70

Supersymmetric SO(10) Models Inspired by Deconstruction

C.-S. Huang, J. Jiang and T.-J. Li

Nucl. Phys. B 702, 109-130 (December 2004).

ANL-HEP-PR-04-66

$\phi$ Meson Production in $\mathrm{Au}+\mathrm{Au}$ and $\mathrm{p}+\mathrm{p}$ Collisions at $\sqrt{s}=200 \mathrm{GeV}$

R.V. Cadman, H.M. Spinka and D.G. Underwood Phys. Rev. Lett.

ANL-HEP-PR-04-123

Technical Compact Essay Encyclopaedic Entry Contributions (10)

C. Zachos

In: Concise Encyclopedia of Supersymmetry and Noncommutative Structures in Mathematics and Physics, edited by S. Duplij, W. Siegel, and J. Bagger

(Kluwer, Dordrecht, 2004) pp. 136, 146, 175, 224, 249, 268, 277, 305, 381, 505.

The Finite Temperature Transition for 2-Flavor Lattice QCD at Finite Isospin Density

J. B. Kogut and D. K. Sinclair

Phys. Rev. D

ANL-HEP-PR-04-64

Transverse Beam Envelope Measurements and the Limitations of the 3-Screen Emittance Method for Space-Charge Dominated Beams

J.G. Power, H.Wang, M.E. Conde, W. Gai, R. Konecny, W. Liu and Z. Yusof

Nuclear Instruments and Methods in Physics Research Section A: Accelerators, Spectrometers, Detectors, and Associated Equipment

ANL-HEP-PR-04-91

Transverse-Momentum Dependent Modification of Dynamic Texture in Central $\mathrm{Au}+\mathrm{Au}$ Collisions at $\sqrt{s}_{\mathrm{NN}}=200 \mathrm{GeV}$

R.V. Cadman, H.M. Spinka and D.G. Underwood

Phys. Rev. Lett.

ANL-HEP-PR-04-124

Transverse Momentum Resummation at Small $\mathrm{x}$ for the Tevatron and LHC

S. Berge, P. N. Nadolsky, F. Olness, and C.-P. Yuan

Phys. Rev. D

ANL-HEP-PR-04-97 
Triggers for RF Breakdown

J. Norem, Z. Insepov and I. Konkashbev

Nuclear Inst. \& Methods in Phys. Research A

ANL-HEP-PR-04-82

Two-Particle Correlations on Transverse Momentum and Minijet Dissipation in $\mathrm{Au}-\mathrm{Au}$ Collisions at $\sqrt{s}_{\mathrm{NN}}=130 \mathrm{GeV}$

R.V. Cadman, H.M. Spinka and D.G. Underwood

Phys. Rev. Lett.

ANL-HEP-PR-04-128

Warped Fermions and Precision Tests

M. Carena, A. Delgado, E. Ponton, T.M.P. Tait, and C.E.M. Wagner

Phys. Rev. D

ANL-HEP-PR-04-88

What Your Next Experiment's Data Will Look Like: Event Stores in the Large Hadron Collider Era

D. Malon

International Journal of Modern Physics A

ANL-HEP-PR-04-111

\section{IV.C. Papers Or Abstracts Submitted To Conference Proceedings}

Advanced Technologies for Distributed Database Services Hyperinfrastructure

A. Vaniachine, D. Malon and M. Vranicar Annual Meeting of the Division of Particles and Fields of the American Physical Society DPF 2004: Int. J. Mod. Phys. A, Riverside, CA, August 26-31, 2004. ANL-HEP-CP-04-107

ATLAS Detector Description Database Architecture

A. Vaniachine, D. Malon and et al Computing in High Energy and Nuclear Physics (CHEP), Interlaken, Switzerland, September 27 - October 1, 2004.

ANL-HEP-CP-04-95

Beyond Persistence: Developments and Directions in ATLAS Data Management D. Malon

Proceedings of the CHEP04 Meeting, Interlaken, Switzerland, September 27 - October 1, 2004.

ANL-HEP-CP-04-101 
Bounds on the Gluino Mass from a Global Parton Density Analysis

E. L. Berger

$12^{\text {th }}$ International Conference on Supersymmetry and Unification of Fundamental Interactions (SUSY 2004), Tsukuba, Japan, June 17-23, 2004.

ANL-HEP-CP-04-75

Branes, Quantum Nambu Brackets, and the Hydrogen Atom

C. Zachos and T. Curtright

Czech. J. Phys.

ANL-HEP-CP-04-71

Determination of the $H W W$ and $H Z Z$ Couplings at the LHC

E. L. Berger

$12^{\text {th }}$ International Conference on Supersymmetry and Unification of

Fundamental Interactions (SUSY 2004), Tsukuba, Japan, June 17-23, 2004.

ANL-HEP-CP-04-79

Differentiating Solutions to the Gauge Hierarchy Problem through Rare Muon Decays

B. Murakami

$12^{\text {th }}$ International Conference on Supersymmetry and Unification of Fundamental Interactions (SUSY 2004), Tsukuba, Japan, June 17-23, 2004.

ANL-HEP-CP-04-85

High Power rf Test on X-band $\mathrm{Mg}_{\mathrm{x}} \mathrm{CA}_{1-\mathrm{x}} \mathrm{TiO}_{3}$ Based Dielectric-Loaded Accelerating Structure.

C. Jing, R. Konecny, W. Gai, S.H. Gold, J.G. Power, A.K. Kinkead and W. Liu

Proceedings of the 11th Advanced Accelerator Concepts Workshop, June 22, 2004, and IEEE Transaction on Plasma Science, Stony Brook, NY.

ANL-HEP-CP-04-92

History and New Ideas for Exotic Particles

H. J. Lipkin

International Workshop on PENTAQUARK04, Spring-8, Hyogo, Japan, 20-23

July 2004.

ANL-HEP-CP-04-103

New Results in Hadron Spectroscopy

H. J. Lipkin

26th Annual Montreal-Rochester-Syracuse-Toronto Conference (MRST 2004 from Quarks to Cosmology), Montreal, Quebec, Canada, May 12-14, 2004.

ANL-HEP-CP-04-52 
Spin Correlations and Velocity-Scaling in NRQCD Matrix Elements

G. T. Bodwin, J. Lee, and D. K. Sinclair

Conference on Quark Confinement and the Hadron Spectrum VI 2004

(QCHS04), Villasimius, Sardinia, Italy, September 21-25, 2004.

ANL-HEP-CP-04-131

Secure Grid Data Management Technologies in ATLAS

M. Branco, D. Malon, and A. Vaniachine

Computing in High Energy and Nuclear Physics Conference (CHEP'04),

Interlaken, Switzerland, 27 September-1 October 2004.

ANL-HEP-CP-04-104

The Description of the ATLAS Detector

A. Vaniachine et al.

Computing in High Energy and Nuclear Physics Conference (CHEP'04), Interlaken, Switzerland, 27 September-1 October 2004.

ANL-HEP-CP-04-105

The Finite Temperature Transition for 3-Flavor Lattice QCD at Finite Isospin Density

J. B. Kogut and D. K. Sinclair

22nd International Symposium on Lattice Field Theory (Lattice 2004), FNAL,

Batavia, IL, June 21-26, 2004.

ANL-HEP-CP-04-69

The Theory of Pentaquarks

H. J. Lipkin

Heavy Quarks and Leptons (HQ\&L 2004), San Juan, Puerto Rico, June 1-5, 2004.

ANL-HEP-CP-04-81

Theory of $W$ and $Z$ Boson Production

P. M. Nadolsky

15th Topical Conference on Hadron Collider Physics (HCP 2004), East

Lansing, MI, June 14-18, 2004.

ANL-HEP-CP-04-138

Upsilon Transverse Momentum at Hadron Colliders

E. L. Berger, J.-W. Qiu, and Y. Wang

Annual Meeting of the Division of Particles and Fields of the American

Physical Society (DPF 2004) Riverside, CA, August 26-31, 2004.

Int'l. J. Mod. Phys. A (World Scientific, 200X).

ANL-HEP-CP-04-110 
The WEB Interface for the ATLAS/LCG MySQL Conditions Databases and Performance Constraints in the Visualisation of Extensive Scientific/Technical Data

A. Vaniachine et al

The Computing in High Energy and Nuclear Physics (CHEP), Interlaken, Switzerland, 27 September--1 October 2004.

ANL-HEP-CP-04-96

Why Pentaquarks are seen in Some Experiments and not in Others

H. J. Lipkin

$32^{\text {nd }}$ International Conference on High Energy Physics (ICHEP 04), Beijing, China, August 16-22, 2004.

ANL-HEP-CP-04-102

\section{D. Technical Reports And Notes}

\section{Technical Reports}

High Energy Division Semiannual Technical Report of Research Activities (January 1, 2004June 30, 2004).

H.M. Spinka, L.J. Nodulman, M.C. Goodman, J. Repond, D.S. Ayres, J. Proudfoot, R. Stanek, T. LeCompte, G. Drake, E.L. Berger, G.T. Bodwin, D.K. Sinclair, C. Zachos, W. Gai and J. Norem

ANL-HEP-TR-04-109

\section{CDF Notes:}

\section{CDF 6919}

Reduced Tracking for Calibration, L. Nodulman, CDF//TRACKING//6919.

\section{$\underline{\text { CDF } 6971}$}

Curvature Corrections for 5.3.1, L. Nodulman, CDF/DOC/ELECTRON/CDFR/6971. 
CDF 7037

Measurement of the $\mathrm{J} / \mathrm{psi}$ Meson and $\mathrm{b}$ Hadron Production Cross $>$ Sections,

M.Bishai, Y.Gotra, J.Kraus, T.LeCompte, J.Lewis, D.Litvintsev, P.Lukens, T.Miao, R.St.Denis, R.Tesarek, S.Tkaczyk, S.Waschke, T.Yamashita, CDF/PUB/BOTTOM/CDFR/7037.

\section{WF Notes:}

$\underline{\text { WF-219 }}$

Measurements of High Brightness Electro Beam

H. Wang, J. Power, W. Liu, W. Gai

January 24, 2004. 


\section{COLLOQUIA AND CONFERENCE TALKS}

\section{Csaba Balazs}

Neutralino Dark Matter

Department of Physics, Northwestern University, Evanston, IL, October 25, 2004.

Stop, Chargino and Neutralino Searches in Run II and at the LHC

TeV4LHC Workshop, Fermilab, Batavia, IL, September 17, 2004.

Neutralino Dark Matter and Electroweak Baryogenesis in the MSSM

Workshop on Strings and the Real World, Aspen, CO, September 10, 2004.

Electroweak Baryogenesis and Neutralino Dark Matter in the MSSM

Victoria Linear Collider Workshop, Victoria, Canada, July 16, 2004.

\section{Puneet Batra}

New Directions for the Little Higgs

Fermilab New Phenomeno Group, Batavia, IL, October 20, 2004.

New Prospects for the Little Higgs and the Electroweak Scale

ANL-HEP Division Lunch Seminar, Argonne, IL, October 12, 2004.

\section{Edmond L. Berger}

Higgs Boson Plus 2 Jet Production: WBF Signal and QCD Backgrounds at NLO"

TeV4LHC Workshop, Fermilab, Batavia, IL, September 17, 2004.

Argonne Theory Institute

LDRD Strategic Initiative Review, ALD Strategic and Programmatic Planning

Council, Argonne National Laboratory, IL, August 13, 2004.

Determinations of the HWW and HZZ Couplings at the LHC and the ILC

Victoria Linear Collider Workshop, Victoria, British Columbia, July 29, 2004.

\section{Geoffrey Bodwin}

Spin Correlations and Velocity-Scaling in NRQCD Matrix Elements

Invited plenary talk, International Workshop on Heavy Quarkonium, Beijing, China, October 12-15, 2004. 
Inclusive Production of Heavy Quarkonium/The NRQCD Factorization Approach and Inclusive Production of Heavy Quarkonium

(A Series of Three Lectures)

Topical Seminar School on Heavy Quarkonia at Accelerators: New Theoretical

Tools and Experimental Techniques, ITP, Academia Sinica, Beijing, China, October 8-11, 2004.

Spin Correlations and Velocity-Scaling in NRQCD Matrix Elements

Invited parallel talk, Sixth International Conference on Quark Confinement and the

Hadron Spectrum, Sardinia, Italy, September 21-25, 2004.

Inclusive Quarkonium Production and the NRQCD Factorization Approach

Joint Series of Theoretical Seminars, Department of Physics, University of Milan, September 16, 2004.

\section{John Campbell}

W+Jets and Heavy Flavor Production

All-D0 Meeting, Fermilab, Batavia, IL, July 23, 2004.

\section{Robert Cadman}

Performance of the STAR Endcap Electromagnetic Calorimeter

The APS Division of Nuclear Physics, October 28, 2004.

\section{Jing Jiang}

New Approaches to SUSY GUT

Physics Department, University of Oregon, Eugene, August 19, 2004.

\section{Kristo Karr}

Experience with POOL in the LCG Data Challenges of three LHC experiments

Integration of POOL into three Experiment Software Frameworks

POOL Development Status and Plans

Contributed to the following three reports by the POOL team for the 2004 CHEP Conference 


\section{Tom LeCompte}

What your Next Experiment's Data Might Look Like: Event Stores in the LHC Era American Physical Society Division of Particles and Fields 2004 Meeting, Riverside, $\mathrm{CA}$

Beyond Persistence: Developments and Directions in ATLAS Data Management

Computing in High Energy and Nuclear Physics 2004 (CHEP’04), Interlaken, Switzerland.

Secure Grid Data Management Technologies in ATLAS

Proceedings of Computing in High Energy and Nuclear Physics 2004 (CHEP’04), Interlaken, Switzerland.

On distributed Database Deployment for the LHC Experiments

Proceedings of Computing in High Energy and Nuclear Physics 2004 (CHEP’04), Interlaken, Switzerland.

ATLAS Detector Description Database Architecture

Proceedings of Computing in High Energy and Nuclear Physics 2004 (CHEP'04), Interlaken, Switzerland.

POOL Integration into Three Experiment Software Frameworks

Proceedings of Computing in High Energy and Nuclear Physics 2004 (CHEP'04), Interlaken, Switzerland.

Experience with POOL in the LCG Data Challenges of Three LHC Experiments

Proceedings of Computing in High Energy and Nuclear Physics 2004 (CHEP'04), Interlaken, Switzerland.

POOL Development Status and Plans

Proceedings of Computing in High Energy and Nuclear Physics 2004 (CHEP'04), Interlaken, Switzerland.

\section{Harry J. Lipkin}

Why Pentaquarks are seen in Some Experiments and not in Others

TRIUMF Laboratory, Vancouver Canada, November 12, 2004.

Why Pentaquarks are seen in Some Experiments and not in Others

University of Washington, Seattle, November 10, 2004.

Direct CP Violation in $B \rightarrow K+\pi$

ANL-HEP Theoretical Physics Seminar, Argonne, IL, September 13, 2004. 
Review of Multiquark States

Nankai University, Tianjin, China, August 26, 2004.

Review of Multiquark States

Institute for High Energy Physics, Beijing, China, August 24, 2004.

Why Pentaquarks are seen in Some Experiments and not in Others

$32^{\text {nd }}$ International Conference on High Energy Physics (ICHEP` 04), Beijing, China, August 16-22, 2004.

What Can We Learn from QCD about Pentaquarks and Vice Versa

RIKEN, Japan, July 29, 2004.

Pentaquarks for Pedestrians

Nagoya University, Japan, July 27, 2004.

History and New Ideas for Exotic Particles

International Workshop on PENTAQUARK04, SPring 8, Hyogo, Japan, July 20-23, 2004.

Coherence, Recoil Effects and Sum Rules in Mossbauer Excitations by Synchrotron Radiation International Workshop on PENTAQUARK04, SPring 8, Hyogo, Japan, July 21, 2004.

\section{Irina Mocioiu}

Very High Energy Neutrinos

Physics Department, Northwestern University, Evanston, IL, November 22, 2004.

Particle Physics and Astrophysics with Very High Energy Neutrinos

Physics Department, Purdue University, West Lafayette, IN, November 2, 2004.

Very High Energy Neutrinos and Cosmic Rays

ANL-HEP Division Lunch Seminar, Argonne, IL, October 5, 2004.

\section{David Morrissey}

Cosmology of the MSSM with a Minimal Singlet

Victoria Linear Collider Workshop, Victoria, Canada, July 29-30, 2004. 


\section{Brandon Murakami}

Differentiating Solutions to the Gauge Hierarchy Problem through Rare Muon Decays California State University, Fresno, July 15, 2004.

Differentiating Solutions to the Gauge Hierarchy Problem through Rare Muon Decays University of California, Irvine, July 13, 2004.

\section{Pavel Nadolsky}

Production of W Bosons in Polarized pp Collisions at RHIC

MIT, Cambridge, MA, October 19, 2004.

Factorization for Transverse Momentum Distributions at Hadron Colliders

ANL-HEP Division Lunch Seminar, Argonne, IL, September 28, 2004.

Transverse Momentum Distributions: Tevatron Experience and LHC

TeV4LHC Workshop, Fermilab, Batavia, IL, September 16-18, 2004.

Resummation for Transverse Momentum Distributions at Hadron Colliders

Department of Physics, Indiana University, Bloomington, IN, September 13, 2004.

\section{James Proudfoot}

The Top Mass Measurement in ATLAS at the LHC, A Test of Systematics. Physics Department, University of Illinois, September 20, 2005.

\section{Geraldine Servant}

First Order Electroweak Phase Transition in the Standard Model with a Low Cutoff Santa Fe 2004 Summer Workshop: "Beyond the Higgs", Santa Fe, NM, August 11, 2004.

First Order Electroweak Phase Transition in the Standard Model with a Low Cutoff: Signatures at the Linear Collider

Victoria Linear Collider Workshop, Victoria, Canada, July 30, 2004.

Warped Dark Matter: Motivations and Signatures

Victoria Linear Collider Workshop, Victoria, Canada, July 30, 2004. 
Warped Unification, Proton Stability and Dark Matter

Physics Department, McGill University, Montreal, Canada, July 5, 2004.

Gauged Baryon Number in Warped GUT

Physics Department, University of Washington, Seattle, June 22, 2004.

Warped Unification, Proton Stability and Dark Matter

Physics Department, Caltech, Pasadena, May 9, 2004.

\section{Donald K. Sinclair}

Simulating Lattice QCD at Finite Temperature and Zero Quark Mass

Workshop on the QCD of Extreme Environments, ANL-HEP, Argonne, IL, July 3, 2004.

\section{Tim Tait}

Summary of the NWU Conference on Z's

D0 Collaboration Meeting, FNAL, Batavia, IL, December 3, 2004.

Basic Lectures on Kaluza-Klein Decomposition

Mini Workshop on Z' Searches, Northwestern University, Evanston, IL, November 16, 2004.

Gauge Extension of the MSSM

Physics Dept., University of California, Berkeley, November 1, 2004.

Gauge Extension of the MSSM

Physics Dept., Purdue University, West Lafayette, IN, October 26, 2004.

High $p_{T}$ Physics in 2015

Proton Driver Workshop, Fermilab, Batavia, IL, October 7, 2004.

\section{Carlos Wagner}

Electroweak Baryogenesis and New Fermions at the TeV Scale

Miami 2004: A Topical Conference on Elementary Particle Physics and Cosmology, Coral Gables and Key Biscayne, FL, December 15, 2004. 
Gauge Bosons in Warped Extra Dimensions and Precision Measurements

Mini-Workshop on Z' Searches, Northwestern University, Evanston, IL, November 16, 2004.

Supersymmetry, Dark Matter and Electroweak Baryogenesis

Astrophysics Group, FNAL, Batavia, IL, September 13, 2004.

New Scenarios for Electroweak Baryogenesis

PASCOS'04 and Pran Nath Fest, Boston, MA, August 17, 2004.

Baryogenesis and New Physics at the Weak Scale

SLAC Summer Institute, Stanford, CA, August 11, 2004.

New Physics at Hadron and Lepton Colliders

Fifth Latin-American Symposium on High Energy Physics, Lima, Peru, July 2004.

Physics Beyond the Standard Model

Lectures at the VI Giambiagi Winter School on Particle Physics, University of

Buenos Aires, Argentina, July 2004.

\section{Cosmas Zachos}

Gauged Skyrmion Masses are Bounded Above

Miami 2004: A Topical Conference on Elementary Particle Physics and Cosmology, Coral Gables and Key Biscayne, FL, December 19, 2004.

Gauged Skyrmion Masses are Bounded Above

ANL-HEP Division Lunch Seminar, Argonne, IL, December 7, 2004. 


\section{HIGH ENERGY PHYSICS COMMUNITY ACTIVITIES}

\section{Csaba Balazs}

Co-Organizer, Argonne Theory Institute on Supersymmetry, Higgs Bosons, and Extra Dimensions, High Energy Physics Division, Argonne, IL, May 9-13, 2005.

Co-Organizer, ANL-HEP Theoretical Physics Seminars, 2004-

\section{Puneet Batra}

Co-Organizer, Argonne Theory Institute on Supersymmetry, Higgs Bosons, and Extra Dimensions, High Energy Physics Division, Argonne, IL, May 9-13, 2005.

Co-Organizer, ANL-HEP Theoretical Physics Seminars, 2004-

\section{Edmond L. Berger}

Co-Chair, International Linear Collider Summer Study, Snowmass, CO, August 13-27, 2005.

Scientific Program Committee, XVIIth International Conference on Particles and Nuclei, PANIC, Santa Fe, NM, October 24-28, 2005.

International Advisory Committee, $3^{\text {rd }}$ International Conference on Flavor Physics, National Central University, Taiwan, October 3-8, 2005.

International Advisory Committee, HADRON 2005, CBPF, Rio de Janeiro, Brazil, August 2126, 2005.

Co-Organizer, Argonne Theory Institute on Supersymmetry, Higgs Bosons, and Extra Dimensions, High Energy Physics Division, Argonne, IL, May 9-13, 2005.

Advisory Board, Frontiers in Contemporary Physics - III, Vanderbilt University, Nashville, TN, May, 2005.

Scientific Program Committee, XXXXth Rencontres de Moriond, "QCD and High Energy Hadronic Interactions", La Thuile, Italy, March 12-19, 2005.

Advisory Committee, 2005 Aspen Winter Meeting on Particle Physics, CO, February 13-19, 2005. 
Organizing Committee, Workshop on QCD, SURA, Washington, DC, February 10-12, 2005.

Scientific Program Committee, Vth Rencontres du Vietnam, Hanoi, Vietnam, August 6-11, 2004.

Scientific Program Committee, Conference on the Intersections of Particle and Nuclear Physics, CIPANP 2006, San Juan, Puerto Rico, May 30--June 3, 2006.

Scientific Advisory Board, Argonne Theory Institute, 2004 — present (http://www.anl.gov/OPA/theoryinstitute/index.html)

Co-Principal Investigator, Argonne Theory Institute, 2003—present

Member, Committee on International Scientific Affairs, American Physical Society, 2003present

Member, Andrew Gemant Award Committee, American Institute of Physics, 2002—present

Member, American Linear Collider Working Group, 2002—present

Adjunct Professor of Physics, Michigan State University, East Lansing, MI, 1997—present.

Member, Coordinated Theoretical-Experimental Project on QCD (CTEQ) Collaboration.

\section{Geoffrey T. Bodwin}

Member, International Advisory Committee and Convener for the Heavy Quarks (and Gluonia) Section, Sixth International Conference on Quark Confinement and the Hadron Spectrum, Sardinia, Italy, September 21-25, 2004.

Editor, Proceedings of the 22nd International Symposium on Lattice Field Theory (Lattice 2004), FNAL, Batavia, IL, 2004--

Convener, Production Section, Quarkonium Working Group QWGNET Proposal to fund a Marie Curie Research Training Network on Heavy Quarkonium, 2003—present.

Member, Working Group for the Production Section of Quarkonium Working Group CERN Yellow Report, November 2002 - present.

Member, Quarkonium Working Group, 2002_present. 


\section{John Campbell}

Co-Organizer, ANL-HEP Theoretical Physics Seminars, 2002-2004.

\section{Jing Jiang}

Co-Organizer, ANL-HEP Division Lunch Seminars, 2002-2004.

\section{Irina Mocioiu}

Co-Organizer, Argonne Theory Institute on Supersymmetry, Higgs Bosons, and Extra Dimensions, High Energy Physics Division, Argonne, IL, May 9-13, 2005.

Co-Organizer, ANL-HEP Division Lunch Seminars, 2004-

\section{Brandon Murakami}

Co-Organizer, ANL-HEP Theoretical Physics Seminars, 2002-2004.

\section{Pavel Nadolsky}

Co-Organizer, Argonne Theory Institute on Supersymmetry, Higgs Bosons, and Extra Dimensions, High Energy Physics Division, Argonne, IL, May 9-13, 2005.

Co-Convener, Hadronic Final States Working Group, XIII International Workshop on Deep Inelastic Scattering (DIS 2005), University of Wisconsin, Madison, WI, April 27-May 1, 2005.

Convener, CTEQ Working Group on W \& Z Boson Physics, 2003

Co-Organizer, ANL-HEP Theoretical Physics Seminars, 2004-

\section{Jim Norem}

Enrico Fermi and the First Chain Reaction, CERN, July 12, 2004. 
Summary of the Argonne Workshop on High Gradient rf

Invited Talk, LINAC 2004, Lubeck, Germany, August 19, 2004.

\section{Geraldine Servant}

Co-Organizer, ANL-HEP Theoretical Physics Seminars, 2003—2004.

\section{Donald Sinclair}

Organizer, Argonne Workshop on QCD in Extreme Environments, Argonne, IL, June 29—July $3,2004$.

Editor, Proceedings of the 22nd International Symposium on Lattice Field Theory (Lattice 2004), FNAL, Batavia, IL, 2004--.

\section{Harold Spinka}

Co-Convenor, STAR Spin Physics Working Group, 2003--.

\section{Tim Tait}

Co-Organizer, Argonne Theory Institute on Supersymmetry, Higgs Bosons, and Extra Dimensions, High Energy Physics Division, Argonne, IL, May 9-13, 2005.

\section{Carlos Wagner}

Co-Organizer, Les Houches Summer School, Les Houches, France, August 2005.

Local Organizer, Kaon 2005 Conference, Northwestern University, Evanston, IL, July 2005.

Co-Organizer, Argonne Theory Institute on Supersymmetry, Higgs Bosons, and Extra Dimensions, High Energy Physics Division, Argonne, IL, May 9-13, 2005.

Organizer, Enrico Fermi Institute Mini-Symposium on Physics Challenges at the LHC, EFI, U Chicago, IL, March 14, 2005.

Member, Argonne HEP Director Search Committee, 2005. 
Scientific Advisory Board, Argonne Theory Institute, October 2004--.

Head, Theory Committee, Argonne National Laboratory, 2003—present

Head, Theory Group, HEP Division, Argonne National Laboratory, September 2002—present

Member, American Linear Collider Group, 2002—present.

Associate Professor, Lecturer for Courses on "Supersymmetry and Advanced Electrodynamics" and "Introduction to Cosmology", EFI, U Chicago, IL, 2000present

\section{Cosmas Zachos}

Member, International Advisory Board, Ninth International Wigner Symposium [WIGSYM-9], Poznan, Poland, July 18-22, 2005.

Member, International Organizing Committee for the Memorial Conference Dedicated to the 80th Birthday of D. V. Volkov, Kharkov, Ukraine, 2005.

Awardee, Collaborative project (GEP1-3327-TB-03) by the US Civilian Research and Development Foundation, jointly with G Jorjadze (Tbilisi, Georgia), quantization of the co-set SL(2,R) WZNW theories, 2004--.

Session Organizer, New Ideas/Developments, 2004 Coral Gables Conference: "Celebrating 40 Years of Quarks and Coral Gables Conferences" [CGC 2004], Key Biscayne, FL, December 15-19, 2004.

Fellow, Institute of Physics, UK, 2004--.

Member, Advisory Panel, J. Phys A: Math Gen (IOP). 


\section{HEP DIVISION RESEARCH PERSONNEL}

\section{Administration}

Price, L.

Hill, D.

\section{Accelerator Physicists}

Conde, $\mathrm{M}$.

Gai, W.

Norem, J.

Yusof, Z.

Power, J.

\section{Experimental Physicists}

Ayres, D.

Proudfoot, J.

Blair, R.

Repond, J.

Byrum, K.

Reyna, D.

Cadman, R.

Spinka, H.

Chekanov, S.

Stanek, R.

Derrick, M.

Talaga, R.

Fields, T.

Tanaka, M.

Goodman, M.

Thron, J.

Hays, E.

Underwood, D.

Kuhlmann, S.

Wagner, R.

LeCompte, T.

Wicklund, A.B.

Magill, S.

Xia, L.

May, E.

Musgrave, B.

Yokosawa, A.

Yoshida, R.

Nodulman, L.

\section{Theoretical Physicists}

Balazc, C.

Murakami, B.

Batra, P.

Nadolsky, P.

Berger, E.

Servant, G.

Bodwin, G.

Sinclair, D.

Campbell, J.

Tait, $\mathrm{T}$.

Jiang, J.

Wagner, $\mathrm{C}$.

Mocioiu, I.

Zachos, C. 


\section{Engineers and Computer Scientists}

Cranshaw, J.

Dawson, J.

Drake, G.

Grudzinski, J.

Guarino, V.

Gieraltowski, J.
Karr, K.

Kovacs, E.

Malon, D.

Schlereth, J.

Vaniachine, A.

\section{Technical Support Staff}

Adams, C.

Ambats, I.

Bulka, J.*

Kasprzyk, T.

Konecny, R.

Cox, G.

Kreps, A.*

Cundiff, $\mathrm{T}$.

Naday, I.*

DeLurgio, P.*

Nephew, T.

Farrow, M.

Reed, L.

Franchini, F.

Rezmer, R.

Skrzecz, F.

Haberichter, W.

Weizeorick, J.*

Hayden, T.*

Wood, K.

\section{Laboratory Graduate Participants}

Beall, E.

Korobkin, D.

Loizides, J.

Miglioranzi, S.
Jing, C.

Morrissey, D.

Wang, $\mathrm{H}$.

\section{Visiting Scientists}

Kovacs, E. (Theory)

Liu, W (AWA)
Lipkin, H. (Theory)

Ramsey, G. (Theory)

Uretsky, J. (Theory)

\footnotetext{
*Under a recent arrangement, the HEP Electronics Group has expanded to provide service to other parts of the Laboratory. About 6 FTE's are devoted to these outside tasks.
} 